\title{
A Feasibility and Optimization Study to Determine Cooling Time and Burnup of Advanced Test Reactor Fuels Using a Nondestructive Technique
}

Jorge Navarro

December 2013

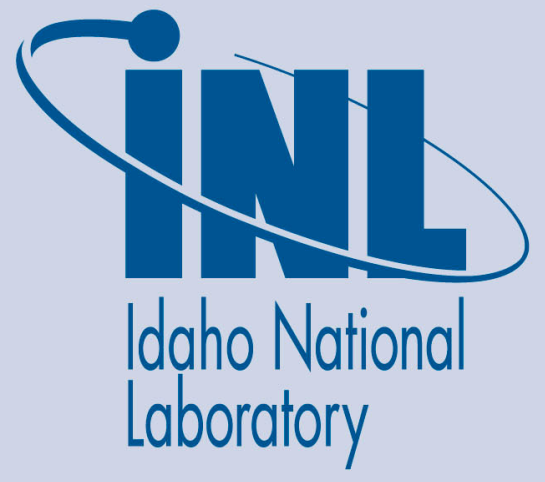

The INL is a U.S. Department of Energy National Laboratory operated by Battelle Energy Alliance 
INL/EXT-13-29997

\title{
A Feasibility and Optimization Study to Determine Cooling Time and Burnup of Advanced Test Reactor Fuels Using a Nondestructive Technique
}

Jorge Navarro

December 2013

\author{
Idaho National Laboratory \\ Idaho Falls, Idaho 83415
}

http://www.inl.gov

Prepared for the

U.S. Department of Energy

Office of Nuclear Energy

Under DOE Idaho Operations Office

Contract DE-AC07-05ID14517 
A FEASIBILITY AND OPTIMIZATION STUDY TO DETERMINE

COOLING TIME AND BURNUP OF ADVANCED TEST

REACTOR FUELS USING A NONDESTRUCTIVE

TECHNIQUE

by

Jorge Navarro

A dissertation submitted to the faculty of The University of Utah

in partial fulfillment of the requirements for the degree of

Doctor of Philosophy

in

Nuclear Engineering

Department of Civil and Environmental Engineering

The University of Utah

December 2013 


\section{The University of Utah Graduate School}

\section{STATEMENT OF DISSERTATION APPROVAL}

The dissertation of

Jorge Navarro

has been approved by the following supervisory committee members:

Terry A. Ring

, Chair

$10 / 15 / 2013$

Date Approved

Tatjana Jevremovic

, Member

$10 / 15 / 2013$

Date Approved

David W. Nigg

, Member

$10 / 15 / 2013$

Date Approved

Sivaraman Guruswamy

, Member

$10 / 15 / 2013$

Date Approved

Hitesh Bindra

, Member

$10 / 15 / 2013$

Date Approved

and by

Micheal Ernest Barber

, Chair of

the Department of

Civil and Environmental Engieering

and by David Kieda, Dean of The Graduate School. 


\begin{abstract}
The goal of this study presented is to determine the best available nondestructive technique necessary to collect validation data as well as to determine burnup and cooling time of the fuel elements on-site at the Advanced Test Reactor (ATR) canal. This study makes a recommendation of the viability of implementing a permanent fuel scanning system at the ATR canal and leads to the full design of a permanent fuel scan system.

The study consisted at first in determining if it was possible and which equipment was necessary to collect useful spectra from ATR fuel elements at the canal adjacent to the reactor. Once it was establish that useful spectra can be obtained at the ATR canal, the next step was to determine which detector and which configuration was better suited to predict burnup and cooling time of fuel elements nondestructively. Three different detectors of High Purity Germanium (HPGe), Lanthanum Bromide ( $\left.\mathrm{LaBr}_{3}\right)$, and High Pressure Xenon (HPXe) in two system configurations of above and below the water pool were used during the study. The data collected and analyzed were used to create burnup and cooling time calibration prediction curves for ATR fuel.

The next stage of the study was to determine which of the three detectors tested was better suited for the permanent system. From spectra taken and the calibration curves obtained, it was determined that although the HPGe detector yielded better results, a
\end{abstract}


detector that could better withstand the harsh environment of the ATR canal was needed. The in-situ nature of the measurements required a rugged fuel scanning system, low in maintenance and easy to control system.

Based on the ATR canal feasibility measurements and calibration results, it was determined that the $\mathrm{LaBr}_{3}$ detector was the best alternative for canal in-situ measurements; however, in order to enhance the quality of the spectra collected using this scintillator, a deconvolution method was developed. Following the development of the deconvolution method for ATR applications, the technique was tested using one-isotope, multi-isotope, and fuel simulated sources. Burnup calibrations were perfomed using convoluted and deconvoluted data. The calibrations results showed burnup prediction by this method improves using deconvolution. The final stage of the deconvolution method development was to perform an irradiation experiment in order to create a surrogate fuel source to test the deconvolution method using experimental data. A conceptual design of the fuel scan system is path forward using the rugged $\mathrm{LaBr}_{3}$ detector in an above the water configuration and deconvolution algorithms. 
To my wife, my family, and all the people with whom I crossed paths and who helped me accomplish this goal. 


\section{TABLE OF CONTENTS}

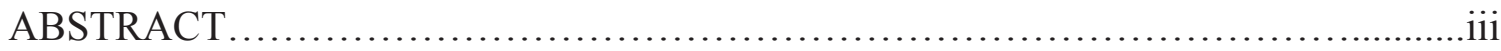

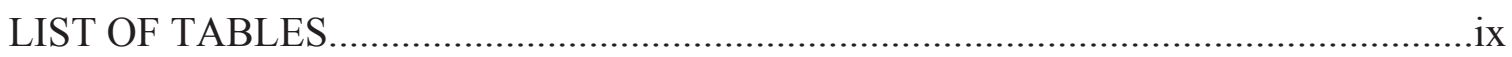

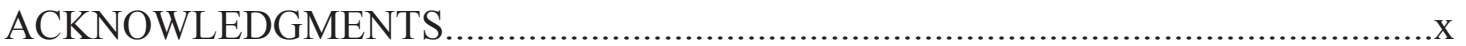

Chapter

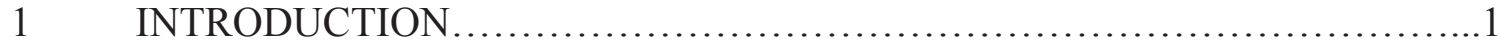

2 A FEASIBILITY STUDY TO DETERMINE COOLING TIME AND THE BURNUP OF ATR FUEL USING A NONDESTRUCTIVE TECHNIQUE AND THREE TYPES OF GAMMA-RAY DETECTORS......................................6

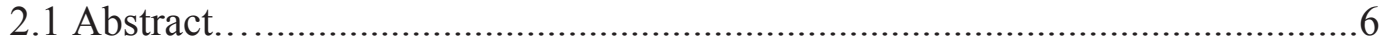

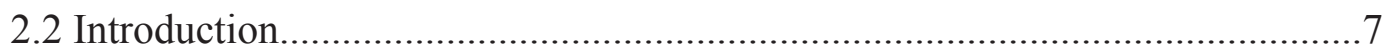

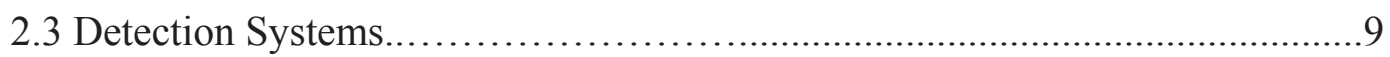

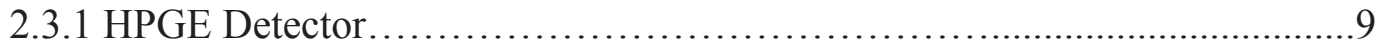

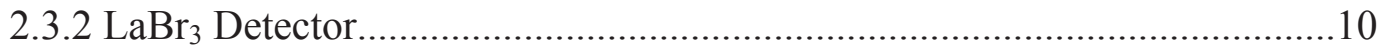

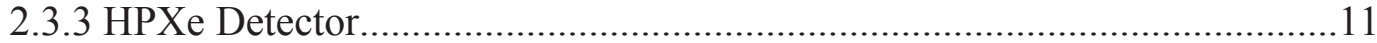

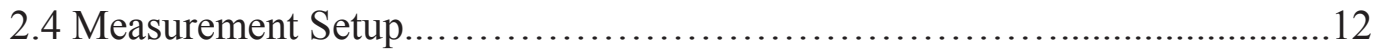

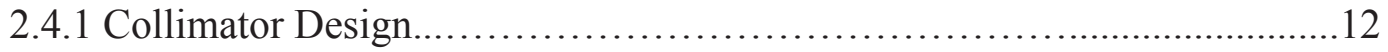

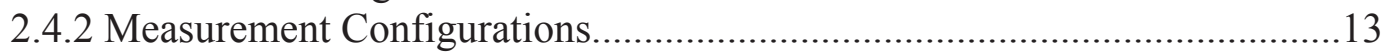

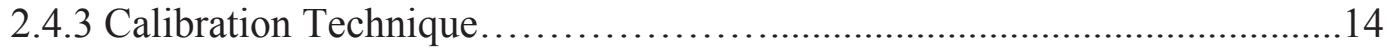

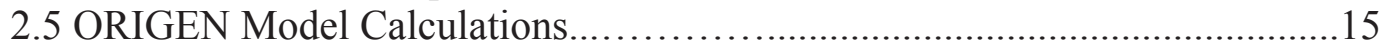

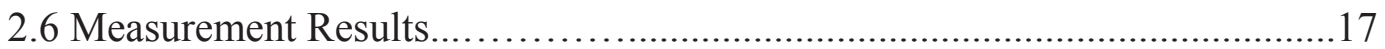

2.6.1 Underwater vs. Above the Water Results....................................................18

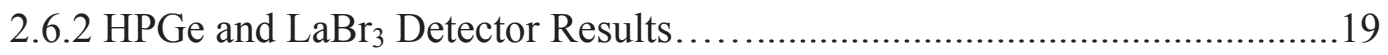

2.6.3 LaBr3 Measurements Results and Comparison with HPGe data...................20

2.6.4 HPXe Gas Detector Measurements Results.............................................21

2.6.5 Burnup Calibration Results...............................................................22

2.6.6 Cooling Time Calibration Results..........................................................24

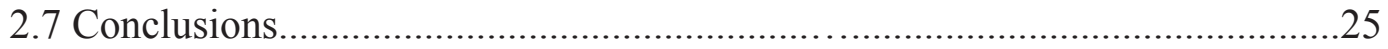

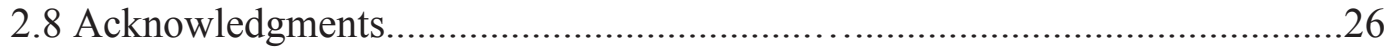

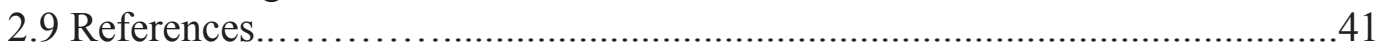

3 GAMMA-RAY DETECTOR SELECTION..............................................43 
GAMMA-RAY SIMULATED SPECTRA DECONVOLUTION OF A LABR3 1"X 1" SCINTILLATOR FOR NONDESTRUCTIVE ATR FUEL BURNUP ON-SITE PREDICTIONS .46

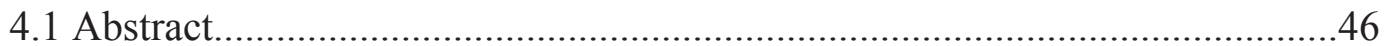

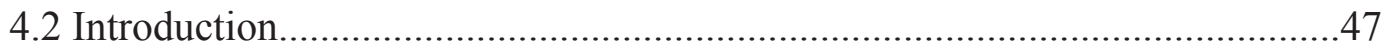

4.3 $\mathrm{LaBr}_{3}$ 1"x1" Scintillator Energy Response Function Determination...............49

4.4 Experimental Measurements.....................................................................50

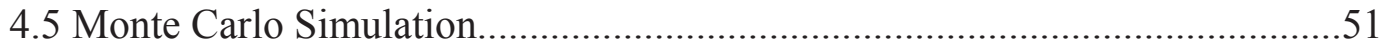

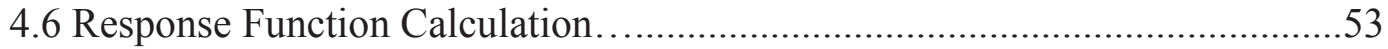

4.7 Spectra Deconvolution...................................................................5

4.8 $\mathrm{LaBr}_{3}$ Spectra Deconvolution of Single Isotopic and Fuel Simulated

Sources using the Maximum Likelihood Expectation Maximization

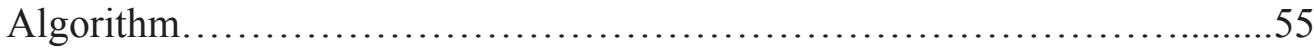

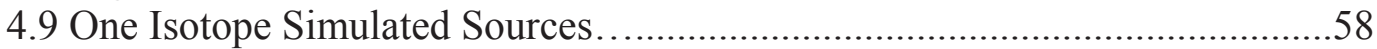

4.10 Fuel Simulated Sources Deconvolution....................................................55

4.11 Use of Deconvolution Spectra for Burnup Determination.........................62

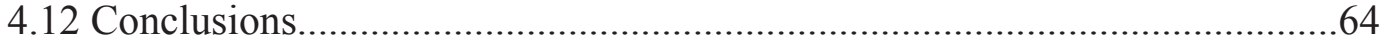

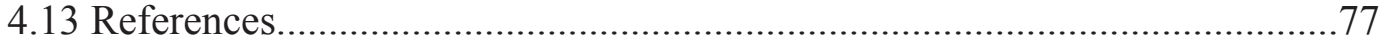

5 GAMMA RAY SPECTRA DECONVOLUTION OF EXPERIMENTAL

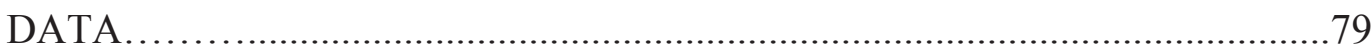

6 MAXIMUM LIKELIHOOD EXPECTATION MAXIMIZATION ALGORITHM SPECTRAL DECONVOLUTON OF A 1"X1" LABR 3 GAMMA-RAY DETECTOR FOR ONE-ISOTOPE, MULTI-ISOTOPE, AND FISSION

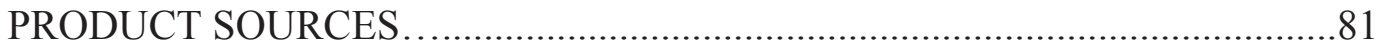

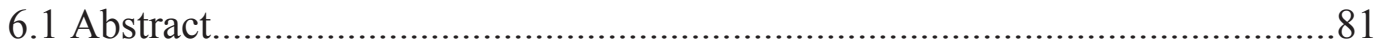

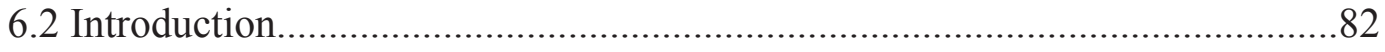

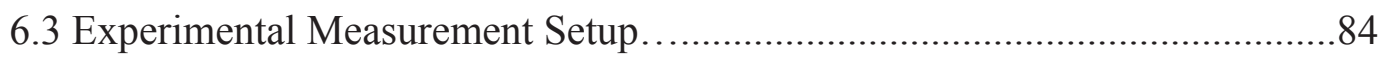

6.4 Detector and Simulation Calibration Measurements...................................84

6.5 Response Function Calculation......................................86

6.6 Maximum Likelihood Expectation Maximization (MLEM) Algorithm......87

6.7 MLEM Deconvolution of One Isotope Source............................88

6.8 MLEM Deconvolution of Multi-Isotope Source..........................89

6.9 Reactor Experiment and High Enriched $\mathrm{LaBr}_{3}$ Detector Measurements........90

6.10 MLEM High Enriched Samples Results................................................91

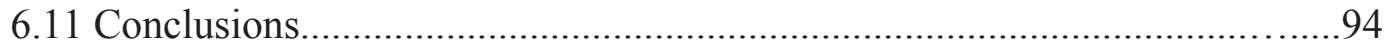

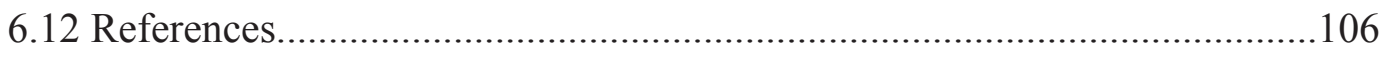

7 PERMANENT ATR FUEL SCANNING SYSTEM PRELIMINARY DESIGN. 
8 SUMMARY, CONCLUSIONS, AND FUTURE WORK...............................110

8.1 Summary and Conclusions............................................................................ 110

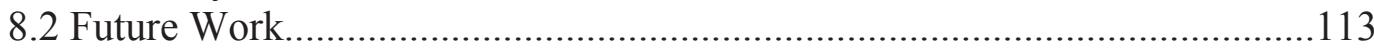

Appendices

A DISTANCE MEASUREMENT DEVICE SELECTION FOR THE ATR FUEL

SCANNING SYSTEM............................................... 116

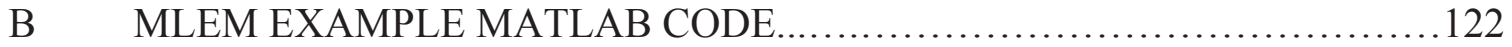

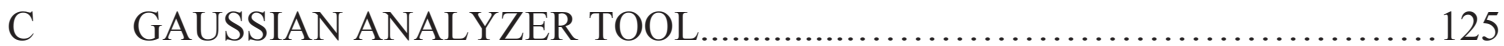

D MCNPX FISSION WIRE RESPONSE FUNCTION SAMPLE CODE.........127

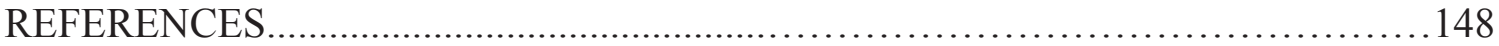




\section{LIST OF TABLES}

Table

Page

2.1 Fission Fragments in Spent Fuel Assemblies...........................................

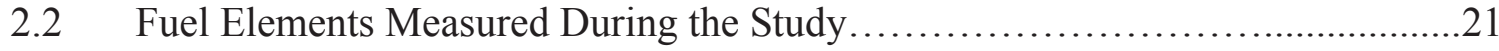

2.3 Gamma-Ray Peaks Used for Calibrations......................................................23

4.1 Gamma Energy Sources used for GEB Parameters........................................51

4.2 Simulated One Isotope Deconvolution Performance Measurements..................63

4.3 Surrogate Fuel Elements Photo-Peaks Resolution............................................ 70

6.1 Gamma Energy Sources used for Gaussian Energy Broadening Parameters........84

6.2 Resolution and Performance Parameters Comparison......................................93

6.3 Resolution Multi-Isotope Source Comparison.............................................96

6.4 Fission Product Multi-Isotope Source Resolution Comparison ..........................100 


\section{ACKNOWLEDGMENTS}

I would like to acknowledge my wife Denise for her patience, encouragement, and support during this long process; I will be eternally grateful for the crossing of our paths. I would also like to thank my mom for always believing that I could do more with my life when I was growing up, even when I did not; to my grandmother Julia, my sisters Ivonne and Mafer, as well as my brother Eduardo and the rest of my family for their support, even when I did not deserve it. I would also greatly like to acknowledge Dr. Terry Ring for his support, counseling, and guidance during this experience. Additionally, I would like to express my gratitude to Dr. David W. Nigg at the Idaho National Laboratory, as well as DOE for giving me the opportunity to work on this project.

I would also like to acknowledge Dr. Steven Howe and the Center for Space Nuclear Research for their support and the creation of the Next Degree Program that allowed me to complete my degree while working in a premiere nuclear facility. Finally I would like to thank Dr. Salvador Hernandez without whose help at the beginning of this journey I would never have been able to be part of the incredible experiences that education has giving me. I am also grateful to anyone who lent me a hand during my educational experience; there are so many people that one way or another contributed to my success that it would be almost impossible to name and thank them all. 


\section{CHAPTER 1}

\section{INTRODUCTION}

The Advanced Test Reactor (ATR) is a uniquely designed experimental reactor located at the Idaho National laboratory. The ATR was first operated in 1967 and has been continuously serving the nuclear community worldwide as a versatile experimental tool. The distinctive four leaf clover design allows for the ATR to have different radiation testing environments in separate regions of the reactor simultaneously. The ATR's main function is to test fuels and materials under intense irradiation conditions; however, the versatility of the ATR also allows for radioisotope production as well as cross-section determination.

The ATR versatility also presents unique operational challenges, which require state-of-the-art simulation and fuel management tools. Currently, there is a new suite of tools that is being developed for ATR operations. In order to validate the new suite of tools, experimental data are needed. A significant element of the validation data required is isotopic burnup of ATR fuel elements. In parallel, there is a need at the ATR facility to develop a technique capable of providing fuel element isotopic information on-site. Acquiring burnup data experimentally can be achieved by destructive and nondestructive techniques. Destructive methods are extremely reliable; however, they are also 
expensive, time-consuming, and carry a potential high dose exposure to the radiation workers as well as not being able to recycle the fuel elements submitted to the test. Nondestructive methods are cheaper, faster, and allow for the reuse of the fuel elements. In order to collect validation data and to explore the feasibility of implementing a fuel scanning system at the ATR canal, this study was initiated. The study consisted of designing equipment, techniques, and testing methodologies to determine if collecting data and using that data to predict burnup is viable.

Chapter 2 of this document presents the feasibility study performed to determine the viability of obtaining meaningful data at the ATR canal, the equipment needed to collect the data on-site, as well as the use of the information collected to predict burnup. The first step of the feasibility study was to determine if it was possible to obtain meaningful gamma spectra at the ATR canal, an environment with high radiation background. The detection system developed consisted of a sealed waterproof detection housing that also acted as shielding for the detectors, collimators, and a sealed pipe (for above water measurements). The detection system was designed to perform either under or above the water measurements. The measurement system was capable of housing three different gamma detectors as well as the electronics associated with each one of them.

The data collected using the three detectors in both configurations (under and above water) were analyzed in order to determine the information quality and usefulness to create nondestructive burnup and cooling time calibrations. Nondestructive techniques to measure fuel burnup and cooling time of fuel elements have been successfully implemented in the past ${ }^{1-3}$. These studies tend to use high performance gamma spectrometers in isolated facilities where the fuel is not in a high background 
environment (HPGE) $)^{4-7}$ and not lower resolution scintillators or gas detectors. However, due to the in-situ nature of the measurement as well as the high radiation background at the ATR canal, two $\mathrm{LaBr}_{3}$ scintillators and a HPXe gas detector were included in this study. $\mathrm{LaBr}_{3}$ detectors have been previously used successfully in studies that require insitu measurements ${ }^{8-10}$ and HPXe detectors can be used in harsh environment applications such as environmental monitoring stations, power reactor fuel measurements, and borehole measurements at nuclear facility sites and in nuclear safeguards ${ }^{11}$. However prior to this study, there was no research focused on using the $\mathrm{LaBr}_{3}$ or HPXe detectors for high enrichment uranium measurements to determine burnup and cooling time of fuel elements.

A number of isotopic ratios and activities have been used as burnup monitors and cooling times ${ }^{6,12,13}$. These isotopic ratios or absolute activities in conjunction with operator-declared values or computer programs can be used to create calibration curves in order to predict cooling time and burnup of irradiated fuel nondestructively and in-situ ${ }^{3,}$ 12,18. The starting point of our study was to use the isotopic ratios and absolute activity isotopes that were reported in the literature ${ }^{14,15,5}$. However, the ATR has a unique serpentine design in which the power can vary greatly from lobe to lobe ${ }^{(16)}$. The irradiation history of each fuel element depends greatly on the position it occupies in each cycle and the type of lobe power to which it is exposed. The variation in irradiation history greatly impacts fuel depletion ${ }^{17}$. The ATR fuel is subject to high fluxes and relatively short cycles (60 days) ${ }^{16,17}$. For those reasons, different isotopes activity ratios than previously reported were also used to create calibration curves that cover the wide variety of cooling times and burnup history of the ATR fuel elements. 
After it was determined that it is feasible to install a permanent fuel scanning system at the ATR canal and before submitting a final design, an optimization of the system was performed. The optimization of the system consisted of selecting a primary detector for the final design. The determination of which detector was better suited for the permanent system is discussed in Chapter 3. This decision could not only be made based solely on the quality of the spectra. The decision also had to weigh in the fact that the detection system will be placed at the ATR canal. Therefore, it must be rugged and need minimal maintenance. The results in the feasibility study showed that the HPGe detector collects the best quality spectra out of the three detectors used in the study. However, the need for HPGe detectors to be operated at cryogenic temperatures, their size, and fragility limit their applications for in-situ measurements. Also, the HPGe detector is not well-suited for hostile environments such as the high radiation field of the ATR canal. Taking the overall performance of the three detectors in addition to their capability to perform well in a hostile environment, it was determine that the $\mathrm{LaBr}_{3}$ detectors are better suited for the ATR permanent fuel scanning system. However, in order to improve the quality of spectra taken with $\mathrm{LaBr}_{3}$ scintillators along with the confidence of the burnup calibration, a spectra enhancement technique was developed and tested.

Deconvolution or enhancement methods are used in gamma spectrometry to improve the quality of spectra collected using lower resolution detectors. Deconvolution when applied properly improves the resolution of each individual photo-peak and allows for a better identification of peaks in the spectra. The development of the deconvolution method for the $\mathrm{LaBr}_{3}$ detector is presented in Chapter 4. This technique consisted of 
calculating the response function of the detector, validating that response using experimental data, implementing the Maximum Likelihood Expectation Maximization algorithm to deconvolve the spectra, as well as developing tools to analyze the performance of the technique. The performance of the method was initially tested using one isotope, multi-istope, and fuel simulated spectra. The study also explored and proved that calibration curves created using simulated fuel improve with deconvolution. After the method was demonstrated to be effective for deconvoluting simulated spectra, an irradiation experiment to produce a highly enriched fission sources was developed and performed with good results. Chapter 6 presents the development of the irradiation experiment as well as the results of the applying the deconvolution method to one, multi, and fission product experimental sources. The final stage of the study presented in Chapter 7 presents a vision of the final design for the permanent fuel scanning system for the ATR canal. 


\title{
CHAPTER 2
}

\author{
A FEASIBILITY STUDY TO DETERMINE COOLING TIME AND \\ BURNUP OF ATR FUEL USING A NONDESTRUCTIVE \\ TECHNIQUE AND THREE TYPES OF GAMMA-RAY \\ DETECTORS
}

\section{$\underline{2.1 \text { Abstract }}$}

The goal of this work was to perform a feasibility study and establish measurement techniques to determine the burnup of the Advanced Test Reactor (ATR) fuels at the Idaho National Laboratory (INL). Three different detectors of High Purity Germanium (HPGe), Lanthanum Bromide $\left(\mathrm{LaBr}_{3}\right)$, and High Pressure Xenon (HPXe) in two detection system configurations of below and above the water pool were used in this study. The last two detectors were used for the first time in fuel burnup measurements. The results showed that a better quality spectra can be achieved with the above the water pool configuration. Both short and long cooling time fuels were investigated in order to determine which measurement technique, absolute or fission product ratio, is better suited in each scenario and also to establish what type of detector should be used in each case for the best burnup measurement. The burnup and cooling time calibrations were established using experimental absolute activities or isotopic ratios and ORIGEN burnup 
calculations. A method was developed to do burnup and cooling time calibrations using fission isotopes activities without the need to know the exact geometry.

\subsection{Introduction}

The new computational models for the Idaho National Laboratory (INL) Advanced Test Reactor will require information on the isotopic burnup of all partially burned fuel elements that are located at the spent fuel canal. The fuel burnup determination will serve as a validation tool when an upgraded ATR 3-D modeling and simulation system is developed in the near future. Although this information may be retrievable from existing records, some confirmatory burnup measurements of fuel elements stored in the ATR canal will be required. Therefore, burnup measurement is a confirmatory tool to compare the results with the existing records and future $3-\mathrm{D}$ model calculations. Also, the techniques developed in this work will be used by ATR reactor operation management to determine burnup of fuel elements routinely and automatically in real time.

An efficient and reliable fuel management program requires having on-site accurate information concerning fuel elements without having to completely rely on computer codes. Nondestructive techniques that can provide burnup and cooling time information of fuel elements are an integral part of any on-site fuel management system. Nondestructive methods are a more attractive tool because they are faster, cheaper, and most importantly, they preserve the integrity of the fuel.

Two of the most important parameters for fuel management are burnup and cooling time since discharge of fuel assemblies. Burnup of the fuel is among the most 
valuable pieces of information that ideally can be obtained by knowing the initial material composition and comparing it to the amount of fissile material left in the fuel at the end of the reactor power cycle. However, performing a procedure that can effectively and directly measure the leftover material is very difficult. The ${ }^{235} \mathrm{U}$ content cannot be directly measured because gamma-rays from the fission products dominate the spectrum by roughly 7 orders of magnitude, which mask the ${ }^{235} \mathrm{U}$ gamma rays. One of the feasible and simpler ways is to use gamma-ray spectroscopy to indirectly estimate burnup by using the spontaneous emission of gamma-rays emitted from the fission products.

The use of gamma-ray spectrometry for the passive, nondestructive determination of spent fuel burnup and cooling time has been common in domestic and international safeguards for decades ${ }^{1-10}$. These techniques usually rely on ratios of various fission products rather than absolute activity measurements. This is because ratios are geometryindependent and can generally be determined more accurately.

There are about 10 major isotopes produced in the fission process that can be measured after fuel discharge. Table 2.1 lists these dominant isotopes, along with their half-lives and gamma-ray energies. Only strong gamma-rays are listed. In this table, the decay half-life, fission yields (per 100 fission disintegrations), Gamma-ray relative intensity (in black), and gamma-ray emission probability (in red) are reported. In addition to the fission product gamma-rays, gamma-rays from the activation of fuel cladding and structural materials such as ${ }^{54} \mathrm{Mn},{ }^{58} \mathrm{Co}$, and ${ }^{60} \mathrm{Co}$ may be also present depending on type of reactor and fuel type. 


\section{$\underline{2.3 \text { Detection Systems }}$}

For fuel burnup measurements, the detection system must be rugged and, if possible, not very sensitive to environmental temperature extremes. Preferably, it should operate at room temperature and must have a good energy resolution. In this feasibility study, we used three types of detectors: HPGe, $\mathrm{LaBr}_{3}$, and HPXe, as shown in Fig. 2.1. This is the first time that the two last detectors were used for fuel burnup measurements. Two different sizes of HPGe detectors (40\% efficient and $25 \%$ efficient) and two different sizes of $\mathrm{LaBr}_{3}(1 " \mathrm{x} 1 "$ and $2 " \mathrm{x} 2 ")$ were utilized. The performance of these detectors has been tested to determine which one is better suited for specific cooling times and burnup. In some cases, e.g., very short cooling time, it was absolutely necessary to use an HPGe detector because of many close-by gamma-ray energies that cannot be resolved using the other two detectors. However, for fuels with a long cooling time, the less expensive $\mathrm{LaBr}_{3}$ or HPXe detector is more likely sufficient.

\subsubsection{HPGE Detector}

HPGe detectors are used for high resolution gamma-ray spectroscopy. A typical energy resolution is about $0.15 \%(2 \mathrm{keV})$ at the $1332 \mathrm{keV}$ line of ${ }^{60} \mathrm{Co}$. With this energy resolution, it is possible to resolve gamma-rays closely spaced in energy $(<2 \mathrm{keV})$. Although their superior energy resolution cannot be matched by other types of detectors, the HPGe detectors must be operated at cryogenic liquid nitrogen temperature. The requirement for cryogenic cooling of HPGe detectors and their overall size limit their range of applications, complicate their implementation and precludes their use in in-situ measurements of spent fuel elements under the water. Also, the HPGe detector cannot be 
used in hostile environments such as in a high radiation field without adequate shielding. In addition, HPGe detectors are very expensive. Recently, new mechanically-cooled HPGe detectors have been developed based on a Stirling-cycle cooler that eliminates the need to use liquid nitrogen for cooling. However, these detectors are even more expensive.

For our measurements, we used two HPGe detectors with detection efficiencies of $45 \%$ and $25 \%$, and both were cooled with liquid nitrogen. Therefore, all measurements with these two detectors were performed above the water, which will be discussed later.

\subsection{2 $\mathrm{LaBr}_{3}$ Detector}

The recently developed $\mathrm{LaBr}_{3}(\mathrm{Ce})$ scintillator is becoming very popular for its use in gamma ray spectroscopy measurements due to its good timing resolution (few hundreds of pico seconds), higher efficiency than a NaI detector of the same size, and most importantly, its energy resolution of 2.8-3.5\% at $662 \mathrm{keV}$ depending on its size and purity. This good energy resolution, in fact, makes this detector an alternative to a HPGe detector in some applications. One major disadvantage is the inherent alpha- and gamma-

contamination arising from decay of ${ }^{227} \mathrm{Ac}$ and ${ }^{138} \mathrm{La}$ impurities in the crystal that cannot be removed completely. These impurities, however, are very small and only affect the low-level gamma-ray counting measurements. Another important factor to consider is the exposure of this detector to high gamma-ray doses of radiation. The most significant change of both yield and energy resolution takes place after the first irradiation with a 0.1 kGy dose. As much as $22 \%$ of the yield is lost; at the same time, the resolution increases from $2.8 \%$ to $4.6 \%$. Therefore, for the ATR fuel burnup measurements depending on 
gamma-ray field, it is necessary to use sufficient shielding if a $\mathrm{LaBr}_{3}$ detector is used. Another factor is its gain change at high count rates and with temperature changes. Temperature stability is important in applications where the detector temperature may change significantly on a daily basis. One way of resolving this issue is to use an internal calibration method for each spectrum taken. This can be done easily in our application because the energies of gamma-rays emitted from fission isotopes for short and long cooling times are very well known.

\subsubsection{HPXe Detector}

The unique properties of this detector are good energy resolution, a wide operational temperature range, and long-term stability against radiation damage, which make it applicable in various fields of fundamental and applied research. This detector can be used in harsh environment applications such as environmental monitoring stations, power reactor fuel measurements, and bore-hole measurements at nuclear facility sites. It can be used for the energy range of 0.1-2 MeV gamma-rays. Unlike $\mathrm{LaBr}_{3}$, the gain of this detector is quite stable over a long period of time. This stability is important in eliminating the need for periodic calibration. The detection efficiency is typically about $\sim 3 \%$ at $662 \mathrm{keV}$ and $\sim 1 \%$ at $1332 \mathrm{keV}$. These low efficiencies to gamma-rays are advantages in a high gamma-ray field that allow us to get the detector closer to the fuel element without saturating the detector and associated electronics. The detector energy resolution at $662 \mathrm{keV}$ is about $2.0-2.5 \%$ and at $1332 \mathrm{keV}$ is about $1.5-2.0 \%$, which is far better than the $\mathrm{NaI}$ detector and a little better than the $\mathrm{LaBr}_{3}$ detector. The main disadvantage of this detector is its small photo-peak efficiency. Overall, this detector is a 
potential alternate to the $\mathrm{LaBr}_{3}$ detector for short cooling time fuels where the radiation field is so high that the $\mathrm{LaBr}_{3}$ detector cannot be placed close to the fuel element or cannot be used at all due to saturation of electronics.

In our measurements, we used a 1.5" in diameter and 4" long HPXe detector made by Constellation Technology. This detector consists of the ionization chamber filled with a Xenon gas plus $0.3 \% \mathrm{H}_{2}$ mixture at the density of $0.35 \mathrm{~g} / \mathrm{cm}^{3}$ and gas pressure of 650 psi (44 atmospheres). This detector has energy resolutions of $2.7 \%$ and $2.0 \%$ for the ${ }^{137} \mathrm{Cs} 662-\mathrm{keV}$ peak and the ${ }^{60} \mathrm{Co} 1332-\mathrm{keV}$ peak, respectively.

\section{$\underline{2.4 \text { Measurement Setup }}$}

\subsubsection{Collimator Design}

Figure 2.2 shows the waterproof housing, collimators, and detector holder used in the measurements. The housing is made of aluminum and is 9" in diameter and 16" long. It consists of cylinder attached to the front and back plates. The back plate has a cables port, which is attached to the PVC tube for sending high voltage (HV) preamplifier and signal cables through and then to electronics modules outside the water pool. Other parts are a support rod made of stainless steel (attached to the front and back plates) and a crane hook for lifting the housing. Inside the housing, there is a holder to center the collimators along the housing cylinder axis. The collimator system is made of bismuth (Bi) that contains several different pieces that can accommodate different types and sizes of detectors. This includes the $40 \% \mathrm{HPGe}, 25 \% \mathrm{HPGe}, 1 " \mathrm{x} 1 " \mathrm{LaBr}_{3}, 2 " \mathrm{x} 2 " \mathrm{LaBr}_{3}$, and HPXe gas detectors used in this study. The collimator system consists of two 2"-thick Bi discs, a collimator insert in the front, and one 1.5"-thick Bi cylinder in the back. Not 
shown in this figure, there is also an additional 0.5 " Bi sleeve that can be inserted inside the bigger cylinder when smaller detectors are used to provide more shielding around the detector. In fact, this Bi sleeve was used in all of our measurements. Different collimator inserts with different shapes and sizes were used to determine which ones deliver the best results under different measurement scenarios.

\subsubsection{Measurement Configurations}

The measurements for $\mathrm{LaBr}_{3}$ and HPXe detectors were performed both in and above the water. However, because of cooling limitations with the HPGe detectors, measurements with these detectors were carried out only with an above the water configuration. Fig. 2.3 shows these two setup configurations used in our measurements. In both configurations, the fuel elements were placed inside the turning table vertically, with the plate number 19 facing the detector. The detector views a small section of the fuel assembly allowed through a small aperture in the collimator insert.

For under water measurements (shown in Fig. 2.3 top photo), the detection system was lowered with the crane to the desire position and ropes attached to the front and back of the lifting rod controlled the position of the detection system. The PVC tube attached to the back plate was used for the cables from the detector to the electronics outside the water pool. In this configuration, it is important to make corrections due to the energydependent attenuation of gamma rays in the water. 


\subsubsection{Calibration Technique}

The absolute gamma-ray peak area of a particular fission product, or the ratio of the particular isotopes, was used to measure the fuel burnup. As will be discussed later, a gamma-ray area for a particular isotope is proportionally related to its activity. This is dependent on the fuel element cooling time. No matter which technique is used, there is a relationship (in most cases linear) between the absolute isotope area or isotopic area ratio and the burnup value. The burnup calibration is obtained by measuring fuel elements with different burnup, well-known irradiation history, and cooling times.

The burnup was then plotted versus the absolute gamma-ray peak area or isotopic gamma-ray area ratio to obtain a linear burnup calibration curve. As long as the burnup calibration for known fuel elements is performed at the same detector to fuel element

distance as the unknown fuel element, using the same gamma-ray energy, the selfattenuation factors in the fuel element and in the water are the same and therefore do not need to be determined. The burnup for the unknown fuel element can be simply derived from the calibration curve.

Absolute activity measurements usually require careful and precise determination of the detection system efficiency calibration. This means that in order to obtain better results, the detection system has to be placed at a precise location at all times. A permanent system capable of positioning fuel elements within 1/1000" accuracy is currently being designed. However, it was not possible in this study with the crane system used. Instead, a technique was developed in which burnup and cooling time calibrations were done at the same distance every time. The reason is that the total net 
area of the gamma-ray peak of interest is directly proportional to absolute activity of fission isotope, as shown in the equation below.

$$
\mathrm{A}=\frac{4 \times \mathrm{Pa} \times \mathrm{D}^{2}}{\mathrm{R}^{2} \times \mathrm{Ip} \times \mathrm{As} \times \mathrm{Aw}}=\mathrm{K} \times \mathrm{Pa}
$$

where:

$\mathrm{A}=$ Isotope activity

$\mathrm{Pa}=$ gamma-ray peak area

$\mathrm{D}=$ Detector to fuel element distance

$\mathrm{R}=$ Detector radius

$\mathrm{Ip}=$ Gamma-ray emission probability

As $=$ Gamma-ray self attenuation factor in the fuel

Aw $=$ Gamma-ray attenuation factor in the water

Parameters D, R, Ip, As, and Aw are all constant so the combination of all can be grouped as a new constant $\mathrm{K}$, as long as burnup and cooling time calibrations are carried out at the same distance. This means the isotope activity is proportional to the gamma-ray peak area.

\subsection{ORIGEN Model Calculations}

ORIGEN 2.2 -is a point depletion and decay computer code used to simulate nuclear fuel cycles ${ }^{11-13}$. In this study,- ORIGEN 2.2 computer code was used to calculate burnup and depletion rates along with fission products isotopes activities of fuel elements. The objective was to linearly correlate measured fuel element isotopic gammaray peak area or gamma-ray peak isotopic ratios with fuel burnup and to predict the isotopic inventory using ORIGEN. The irradiation history needed in order for ORIGEN 
to calculate burnup, isotopic inventory, and decay of the ATR fuel elements was obtained from PDQ ${ }^{14}$ results (average fuel element power) and operator-declared values (lobe power, effective power days, and element cooling time). PDQ is a two-dimensional group diffusion computer code that employs continuous slowing of neutrons and is the standard code to calculate cycle operations at the Advanced Test Reactor Facility.

The fission product ratios measured by gamma-ray spectrometry can be directly compared with those computed by a validated isotope build up and decay computer code. Correlations between fission products activities or ratios with burnup of different types of fuel (mainly long cooling times) have been the topic of several studies ${ }^{3,7}$. In these studies, burnup was either an operator-declared value or a value calculated using computer codes (i.e., ORIGEN). Based on those previous studies ${ }^{1,3,7}$, several calibration curves using the burnup results in combination with experimental measurements of fission products absolute and isotopic ratio activities were created. Correlations are necessary because determining burnup directly from gamma spectroscopy is not possible. Establishing consistent relationships between gamma-ray peak area measurements and burnup will ultimately allow experimental determination of ATR fuel elements burnup without the need of particular element irradiation history or computer calculations.

The main objective of the feasibility study was to find correlations between experimental measurements and burnup. The fuel elements selection was based primarily on analyzing ORIGEN results from several calculations performed on a series of elements with a wide range of burnup and cooling times and irradiation histories. The process was also focused on finding sets of elements for which their burnup and area or gamma-ray peak area ratios yield linear correlations. In addition, the results from the 
ORIGEN calculations gave information on which fission products isotopes to expect and their relative strength for different elements cooling times

\subsection{Measurement Results}

The ATR is a highly-heterogeneous light-water and beryllium moderated, beryllium reflected, light-water cooled system powered with highly enriched fuel (93\% $\left.{ }^{235} \mathrm{U}\right)$ [16]. The ATR fuel elements have an average initial content of 1073 grams of ${ }^{235} \mathrm{U}$. Each fuel element is typically burned for two or three noncontiguous cycles during its useful lifetime. Typical operating cycle lengths are in the range of 45-60 days. Due to the unique design of the ATR, the power level in each corner lobe of the reactor can be controlled independently ${ }^{17}$, potentially exposing the fuel elements to different power and flux levels in each fuel cycle.

The elements measured in this study are shown in Table 2.2, along with their average element power, initial ${ }^{235} \mathrm{U}$ content, and amount of ${ }^{235} \mathrm{U}$ at the time of the measurements. The measurements were performed at the canal adjacent to the reactor using five different gamma detectors. The fuel elements were placed into a turntable fuel holder with plate number 19 facing towards the detector. Fig. 2.4 shows the ATR fuel assembly placed in the turntable (left) and the fuel cross-section of 19 plates (right). The fuel assembly is 4 feet long.

The isotopes and associated gamma-ray peaks used in this study are shown in Table 2.3. The counting time for each measurement was 1000 seconds. The spectral analysis was performed using SPEX ${ }^{15}$ software. This program was used to analyze the isotopic information given by each spectrum and to perform Gaussian fits to single and 
multiple peaks. SPEX calculated the net areas of the fitted peaks along with their uncertainties. The measurement uncertainties vary depending on the strength of the gamma-ray peak; however the typical net area uncertainty is around $5 \%$.

\subsubsection{Underwater vs. Above the Water Results}

Underwater and above water measurements were made using the $\mathrm{LaBr}_{3}$ detectors. The main goal of these measurements was to determine which setup (under water or above water) would be a better choice for building a future permanent system. $\mathrm{The} \mathrm{LaBr}_{3}$ detector was chosen because it was not possible to place the HPGe detector in the water due to the liquid nitrogen cooling system. The goal was to compare the spectra of above and below water measurements. Figs. 2.5 and 2.6 are spectra of element XA374T taken with the same detector in two different configurations of above and below water, respectively. The detection system in the underwater measurements was placed at $4.0 \mathrm{ft}$ from fuel element XA374T.

As mentioned before, the detection system for above water measurement included a 14-foot pipe attached to the front of it and the end of the pipe was 6 inches from the fuel element. By comparing the two spectra, it is very clear that quality of the gamma-ray spectrum, peak-to-Compton $(\mathrm{P} / \mathrm{C})$ ratio, is much better for the above the water measurement, especially for low energy gamma-rays, e.g., $605 \mathrm{KeV}$ of ${ }^{134} \mathrm{Cs}$ and $662 \mathrm{keV}$ of ${ }^{137} \mathrm{Cs}$ peaks. This is mainly due to the fact that gamma-rays are severely attenuated going through the water in the underwater measurement. Gamma attenuation is higher in the underwater detector setup due to 4 feet of water that gamma-rays have to travel before reaching the detector. While in the above water setting, gamma-rays need to 
travel through only 6 inches of water. Another important distinction between these two spectra is the existence of the hydrogen neutron-capture peak at $2223 \mathrm{keV}$ in the under the water measurement (see Fig. 2.6). This peak was not observed in the above water spectrum due to the shorter distance between the fuel element and pipe (less water between), and also due to the long 14 feet of air within the collimated pipe through which this gamma-ray has to travel to reach the detector.

\subsubsection{HPGe and $\mathrm{LaBr}_{3}$ Detector Results}

The results of the measurements taken with high purity germanium and $\mathrm{LaBr}_{3}$ detectors were divided into three groups, based on their cooling time. The first group contains measurements made on element XA815T that was in the reactor for one cycle (about 60 days). Element XA815T was monitored with the high purity germanium detector for 2 months. Measurements were taken every two weeks to monitor the decay history of the fission product isotopes. Fig. 2.7 top spectrum shows a spectrum taken with a $45 \%$ efficient HPGe detector after 38 days of cooling time. Due to the high resolution of this detector, the peaks of all the isotopes are very well separated. It can be seen that the spectrum is dominated by the gamma-ray emitted from the short-lived fission product isotope of ${ }^{140} \mathrm{La}$ with a half-life of 12 days.

The second group of experiments consisted of measurements done on element XA826T, during a 5-month span. This element (XA826T) had been in the reactor for one cycle. In Fig. 2.7, the middle spectrum shows the data taken from element XA826T using the HPGe detector. The cooling time was 186 days when this measurement was carried out. This spectrum when compared to the top spectrum in Fig. 2.7 shows the natural 
decay behavior of the fuel fission products. The spectrum is dominated by the ${ }^{95} \mathrm{Zr}(64-$ day half-life) and ${ }^{95} \mathrm{Nb}$ (35-day half-life) peaks while the ${ }^{140}$ La peaks are mostly decayed away. It can also be seen that the long-lived ${ }^{137} \mathrm{Cs}$ and ${ }^{134} \mathrm{Cs}$ are starting to show up in the spectrum. Other dominating peaks are from ${ }^{144} \mathrm{Ce}$ (284-day half-life). Some of peaks can be also seen in the top spectrum of Fig. 2.7 but due to the dominate role of ${ }^{140} \mathrm{La}$ isotope, these are relatively weak peaks.

The third group of experiments consisted of measurements done on several elements with longer cooling times ( 6 months-3.5 years). Fig. 2.7 bottom spectrum shows the HPGe data taken for element XA374T, which had been in the reactor for 3 cycles with the cooling time of 836 days. Comparing this spectrum with the middle and top spectra indicates that almost all the short-lived isotopes $\left({ }^{140} \mathrm{La},{ }^{95} \mathrm{Zr}\right.$, and $\left.{ }^{95} \mathrm{Nb}\right)$ have decayed away. Most of the peaks in Fig. 2.7 (bottom) are due to long-lived isotopes. The strongest peaks come from ${ }^{134} \mathrm{Cs},{ }^{137} \mathrm{Cs}$, and ${ }^{144} \mathrm{Ce}$. Also, in this spectrum, the ${ }^{154} \mathrm{Eu}$ gamma-ray peaks can be seen. From these spectra, it is clear that all close-by gamma-rays are easily resolved due to the high energy resolution of the HPGe detector.

\subsection{3 $\mathrm{LaBr}_{3}$ Measurements Results and Comparison with the HPGe Data}

The measurements on the three fuel elements mentioned above were also performed using the $\mathrm{LaBr}_{3}$ detector above the water. The spectra taken with the $\mathrm{LaBr}_{3}$ are shown in the Fig. 2.8. Here again, the top spectrum corresponds to very short cooling time fuel (25 days), middle spectrum is for short cooling time fuel (173 days), and bottom spectrum is for long cooling time fuel ( 824 days). The comparison between these two 
detectors was made to determine which apparatus was better suited for a permanent system.

By comparing the HPGe spectra (Fig. 2.7) with the $\mathrm{LaBr}_{3}$ spectra (Fig. 2.8), it is clear that the HPGe spectrum has more peaks and that the close-by peaks are easier to resolve. Being able to resolve close-by peaks becomes very important for short cooling time fuels. In addition, the $\mathrm{P} / \mathrm{C}$ ratio is much better for the $\mathrm{HPGe}$ detector, as expected, resulting in a better area definition and reducing the measurement uncertainties. However, the results show that a $\mathrm{LaBr}_{3}$ detector can be used for burnup measurements as well, when the cost of the HPGe detector is prohibitive or in high radiation environments when very short cooling time fuel elements are measured.

\subsubsection{HPXe Gas Detector Measurement Results}

A high pressure xenon detector was also used in this study and the spectra taken with the three fuels mentioned above are shown in Fig. 2.9. This detector was selected because it has lower efficiency than the HPGe and $\mathrm{LaBr}_{3}$ detectors and therefore can be placed very close to the fuel $(<2$ inches) and also is the only candidate for very hot fuels ( $<1$ month cooling time) when the other two detectors cannot be used. Its disadvantage is the fact that the majority of gamma-ray interactions occur through Compton scattering; only a few of them contribute to the full-energy peaks. Therefore, the peak-to-Compton ratio is very small and therefore weaker peaks will be buried in Compton background that cannot be resolved, as seen in Fig. 2.9, in comparison with the HPGe (Fig. 2.7) and $\mathrm{LaBr}_{3}$ (Fig 2.8) spectra. 


\subsubsection{Burnup Calibration Results}

Gamma-ray spectroscopy is a well-researched and reliable nondestructive method that can be used to estimate fuel element burnup and cooling time for fuel assemblies with short to long cooling times. This technique uses the measured gamma-ray signatures and correlates either an absolute or isotopic ratio of fission products from the irradiated fuel elements with known values for burnup and cooling times. Choosing between absolute or ratio measurements depends on the type of fuel, cooling time, geometry, and efficiency of the detector.

Developing a technique to create high enriched ATR fuel burnup and cooling time calibration curves was a high prority objective of this feasibility study. In order to develop a calibration technique for the ATR fuel, data taken with five different detectors were analyzed. The analysis was focus on creating burnup and cooling time calibration curves from experimental absolute gamma-ray peak area and gamma-ray peak isotopic area. There were a number of calibrations curves made during this study, some of them based on previously reported results ${ }^{3}$, but also some new curves were created based on trends found during analysis of the data. From the analysis, it was deduced that there are three gamma-ray peak area ratios and two gamma-ray peak net-areas that are consistent burnup and cooling time monitors for ATR fuel elements. With the new technique developed for the ATR fuel, we were able to create burnup calibrations curves using gamma-ray peak areas and gamma-ray peak ratios, without the need to know the exact geometry of the system as long as the relative distance of the fuel elements remains constant for each calibration curve. In addition, to reduce uncertainties, a permanent detection system is needed. The permanent system will allow us to know the exact 
location of each measurement and take the data for a longer period of time in order to improve signal-to-noise of the spectra collected.

The most consistent monitor was the area ratio ${ }^{134} \mathrm{Cs} /{ }^{137} \mathrm{Cs}$. The results can be seen in Fig. 2.10 for HPGe (top), HPXe (middle), and $\mathrm{LaBr}_{3}$ (bottom) detectors, where experimental isotopic ratios for five long cooling time elements were plotted against burnup calculated with ORIGEN. In this figure, it can be seen that data points together with their uncertainties fall into the linear trend. The other area ratio that was consistent when used as a burnup monitor was ${ }^{134} \mathrm{Cs} /{ }^{144} \mathrm{Ce}$. This ratio was not previously reported. This was also corroborated with a second set of data measured. The results for the ${ }^{134} \mathrm{Cs} /{ }^{144} \mathrm{Ce}$ area ratio as a burnup monitor using the HPGe detector is shown in Fig. 2.11 top plot.

Comparisons were made between the burnup calibration curves obtained with the HPGe and $\mathrm{LaBr}_{3}$ detectors to determine which detector data yields the better linear fit and the measured uncertainties associated with it. By analyzing several calibration curves for each detector, it was determine that in general, the calibration curves created using HPGe data gave a better fit and had lower uncertainties, as expected. Plots in Fig. 2.10 (top) and Fig. 2.10 (bottom) are an example of calibration curves created with data from an HPGe and $\mathrm{LaBr}_{3}$ detector, respectively. The uncertainties in the HPGe calibration curve are smaller than the uncertainty bars in the $\mathrm{LaBr}_{3}$ plot. For the reasons stated above, and also because the above the water detection setup yields better results, the HPGe detector was selected as the primary detector for the future permanent system. However, we are planning to use the 1"x1" $\mathrm{LaBr}_{3}$ detector for longer cooling time fuels (1-3 years) as well. 
This detector is a less expensive alternative to HPGe detector that works well for the longer cooling time fuels.

Finally, the plots using absolute peak areas of gamma rays emitted from ${ }^{134} \mathrm{Cs}$ and ${ }^{137} \mathrm{Cs}$ isotopes as burnup monitors showed a linear relationship with the burnup. These peak areas are proportional to the isotopes activities if measurements are done at the same detector to fuel element distance. Fig. 2.11 (bottom) shows the burnup calibration plot for the ${ }^{134} \mathrm{Cs}$ isotope. Not all points fall exactly on the line. This can be attributed to not being able to exactly control the distance between the fuel elements and the detection system during measurements.

\subsubsection{Cooling Time Calibration Results}

We also investigated the possibility of using experimental absolute gamma-ray peak area or isotopic gamma-ray peak area ratios as cooling time monitors. The results are depicted in Fig. 2.12 using the HPGe detector, which shows the ${ }^{144} \mathrm{Ce} /{ }^{137} \mathrm{Cs}$ ratio, ${ }^{95} \mathrm{Zr}$, and ${ }^{144} \mathrm{Ce}$ activities can be used as good cooling time predictors. The ${ }^{144} \mathrm{Ce} /{ }^{137} \mathrm{Cs}$ area ratio was the most consistent and efficient when correlated with ATR operatordeclared cooling time. The top plot shows the ${ }^{144} \mathrm{Ce} /{ }^{137} \mathrm{Cs}$ ratio as a function of cooling time. It is clear that all points representing ATR fuel elements and their uncertainties fall on the line. Middle and bottom plots show the correlations obtained by using ${ }^{95} \mathrm{Zr}$ and ${ }^{144} \mathrm{Ce}$ activities and operator-declared cooling times values. It can be seen that all points along with their uncertainties follow a linear trend. In addition, we also looked at the ${ }^{95} \mathrm{Nb}$ isotope and the results show exactly the same trend as ${ }^{95} \mathrm{Zr}$ isotope. We believe that with the permanent system the uncertainties can be reduced substantially. 


\section{$\underline{2.7 \text { Conclusions }}$}

We have established several burnup calibrations for ATR fuel elements using $\mathrm{LaBr}_{3}, \mathrm{HPGe}$ and HPXe detectors. The results showed that the $\mathrm{LaBr}_{3}$ and HPXe detectors can be used for burnup measurements. We found that for highenriched fuels, there is a consistent linear relationship between the ${ }^{134} \mathrm{Cs} /{ }^{137} \mathrm{Cs}$ gamma-ray peak area ratio and burnup for all three detectors. The analysis of the data also concluded that for ATR fuel elements, the area ratio ${ }^{134} \mathrm{Cs} /{ }^{144} \mathrm{Ce}$ has a linear relationship with burnup. The use of this ratio has not previously been reported as a burnup monitor. Also it is important to state that although the detection system was not set up for absolute activity measurement, a new method was develop to determine burnup and cooling time of ATR fuel elements using experimental isotopic gamma-ray peak area, as well as isotopic gamma-ray peak area ratios without a need for precise absolute detector efficiency.

The gamma-ray peak area of some fission products, among them ${ }^{134} \mathrm{Cs}$ and ${ }^{137} \mathrm{Cs}$ isotopes, was found to have a linear relationship with burnup and therefore they can be used as monitors for ATR fuel. We also investigated which other isotopic ratios and absolute activities can be used as ATR fuel cooling time monitors and it was determined that ${ }^{144} \mathrm{Ce} /{ }^{137} \mathrm{Cs}$ ratio, ${ }^{144} \mathrm{Ce},{ }^{95} \mathrm{Zr}$, and ${ }^{95} \mathrm{Nb}$ showed a linear relationship as a function of cooling time for ATR fuel elements. These results indicate that the detection system is performing very well and that using an above water configuration is better suited for the ATR environment and needs, making it the recommended option to build the future permanent system. Our future plan is to design and construct an automatic permanent system capable of measuring fuel burnup in real-time. 


\subsection{Acknowledgements}

This work was supported by the U.S. Department of Energy (DOE) under Battelle Energy Alliance, LLC Contract No. DE-AC07-05ID14517. We would also like to thank Robert Austin and Constellation Technology Corporation for providing the HPXe detector used in this work. 
Table 2.1 Fission fragments in spent fuel assemblies with their half-lives and associated main gamma rays from their decay. The $\%$ relative intensities are in black and the gamma-ray emission rates per 100 decays are in red. Only strong gamma-rays are listed in this table.

\begin{tabular}{|c|c|c|c|}
\hline Isotope & $\begin{array}{l}\text { Half- } \\
\text { Life }\end{array}$ & $\begin{array}{l}\text { Fission } \\
\text { Yields } \\
(\%)\end{array}$ & Main gamma rays (keV) \\
\hline $\begin{array}{l}{ }^{140} \mathrm{Ba} \rightarrow \\
{ }^{140} \mathrm{La} \\
{ }^{140} \mathrm{La} \rightarrow \\
{ }^{140} \mathrm{Ce}\end{array}$ & $\begin{array}{l}12.75 \mathrm{~d} \\
1.68 \mathrm{~d}\end{array}$ & 6.2 & $\begin{array}{c}537.26(100 \%, 24.4 \%) \\
328.8(19.6 \%, 20.3 \%), 487.0(44.7 \%, 45.5 \%), 815.8 \\
(24.2 \%, 23.3 \%) \\
925.2(7.2 \%, 6.9 \%), 1596.2(100 \%, 95.4 \%), 2521.4 \\
(3.59 \%, 3.5 \%)\end{array}$ \\
\hline${ }^{95} \mathrm{Zr}$ & $64.02 \mathrm{~d}$ & 6.5 & $724.2(80.6 \%, 44.2 \%) ; 756.7(100 \%, 54.5 \%)$ \\
\hline${ }^{95} \mathrm{Nb}$ & $34.99 \mathrm{~d}$ & 6.5 & $765.8(100 \%, 99.8 \%)$ \\
\hline${ }^{144} \mathrm{Ce}$ & $284.5 \mathrm{~d}$ & 5.5 & $\begin{array}{c}133.5(804 \%, 11.9 \%) 696.4(100 \%, 1.3 \%) ; 1489.2 \\
(21.4 \%, 0.3 \%) ; \\
2185.6(57 \%, 0.7 \%)\end{array}$ \\
\hline${ }^{103} \mathrm{Ru}$ & $39.27 \mathrm{~d}$ & 3.0 & $497.1(100 \%, 91.0 \%) ; 610.3(7.4 \%, 5.8 \%)$ \\
\hline${ }^{106} \mathrm{Ru}$ & $1.02 \mathrm{yr}$ & 0.4 & $\begin{array}{c}511.4(100,20.4 \%) ; 621.9(48.8 \%, 9.9 \%) ; 873.5(7.6 \%, \\
0.4 \%) ; \\
1050.5(7.6 \%, 1.6) ; 1128.1(1.98 \%, 0.4 \%) ; 1562.2(0.8 \%, \\
0.2 \%)\end{array}$ \\
\hline $\begin{array}{l}{ }^{133} \mathrm{Xe} \rightarrow \\
{ }^{133} \mathrm{Cs}+\mathrm{n} \\
\rightarrow{ }^{134} \mathrm{Cs}\end{array}$ & $2.06 \mathrm{yr}$ & $6.7^{\mathrm{a}}$ & $\begin{array}{c}569.3(15.3 \%, 15.4 \%) ; 604.7(100 \%, 97.6 \%) ; 795.8(87 \%, \\
85.5 \%) ; \\
801.8(8.8 \%, 8.7 \%) ; 1038.5(1.1 \%, 1.0 \%) ; 1167.9(2.0 \%, \\
1.8 \%) ; \\
1365.1(3.3 \%, 3.0 \%)\end{array}$ \\
\hline $\begin{array}{c}{ }^{154-\mathrm{xn}} \mathrm{Eu}+ \\
{ }^{\mathrm{xn}} \underset{\mathrm{154}}{\rightarrow} \mathrm{Eu}\end{array}$ & $8.59 \mathrm{yr}$ & $1.6^{\mathrm{b}}$ & $\begin{array}{c}123.0(100 \%, 40.6 \%) ; 247.9(16.8 \%, 6.9 \%) ; 996.3(30.3 \%, \\
10.5 \%) ; \\
1004.8(50.5 \%, 17.9 \%) ; 1274.4(95.0 \%, 35.0 \%)\end{array}$ \\
\hline${ }^{137} \mathrm{Cs}$ & $\begin{array}{c}30.07 \\
\mathrm{yr}\end{array}$ & 6.2 & $661.6(100 \%, 85.1 \%)$ \\
\hline
\end{tabular}

a) Fission yield is for the ${ }^{133} \mathrm{Xe}$ isotope

b) Fission yield is for the ${ }^{153} \mathrm{Eu}$ isotope 


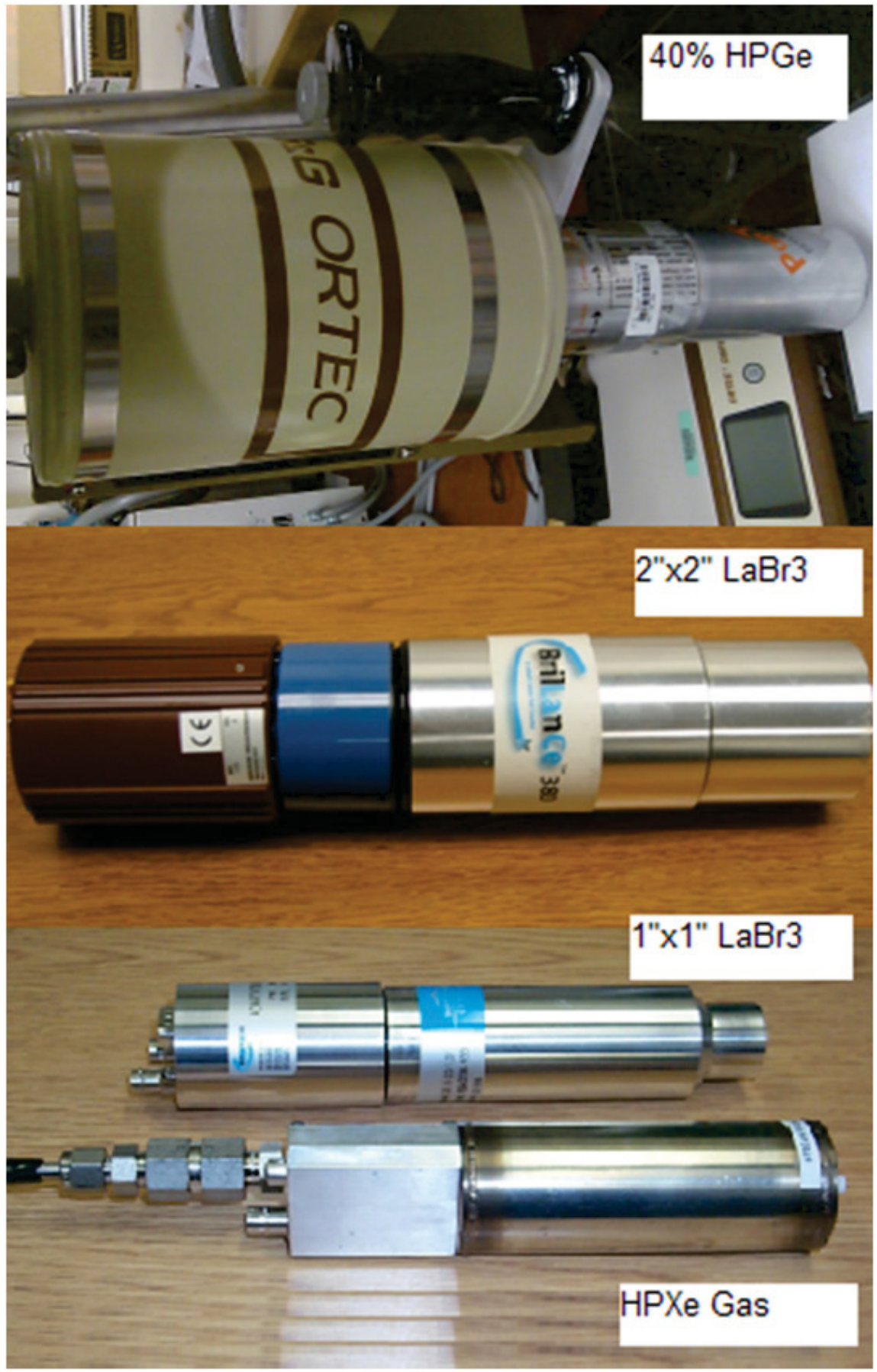

Figure 2.1 Detectors used in the study 


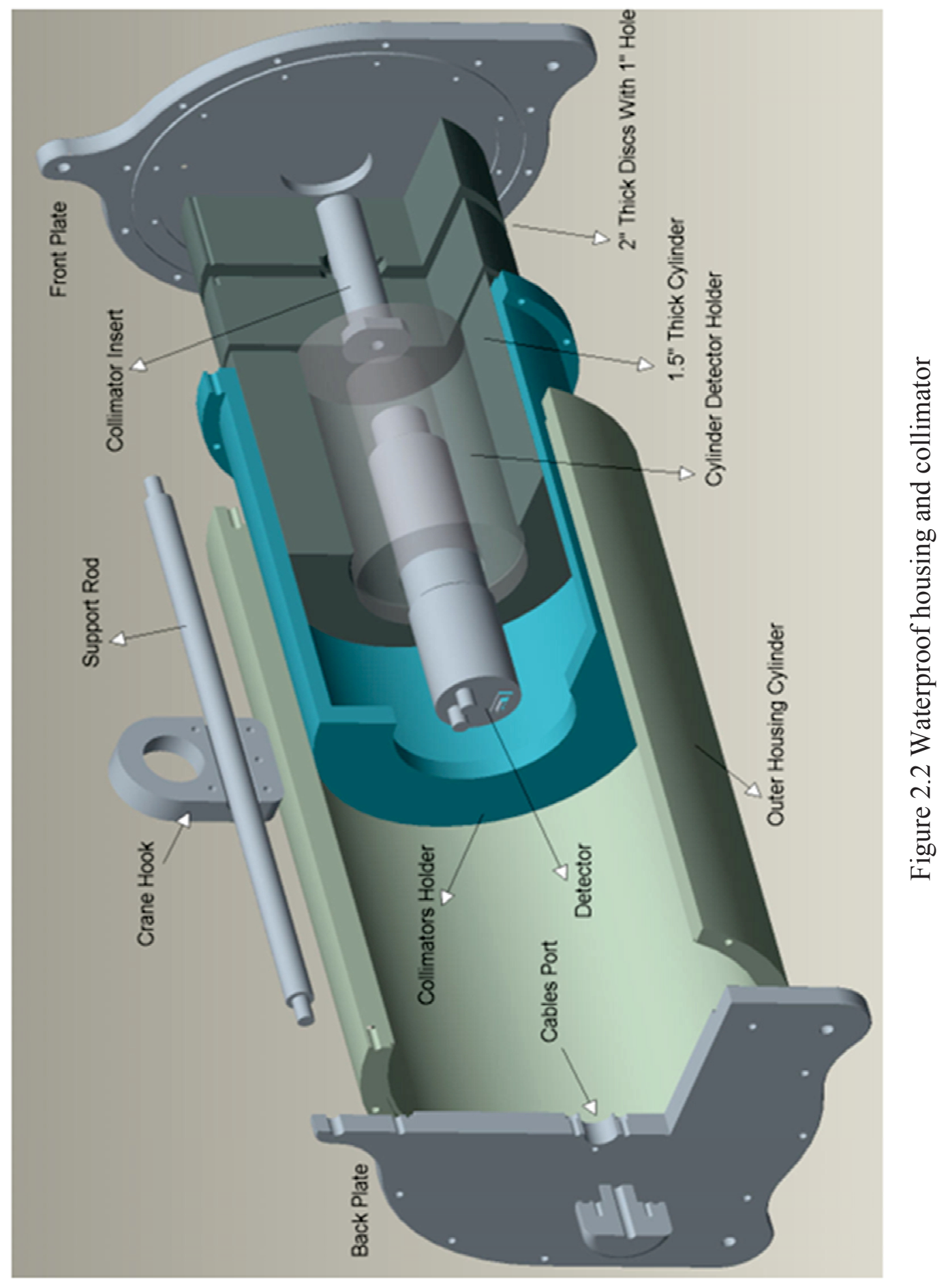




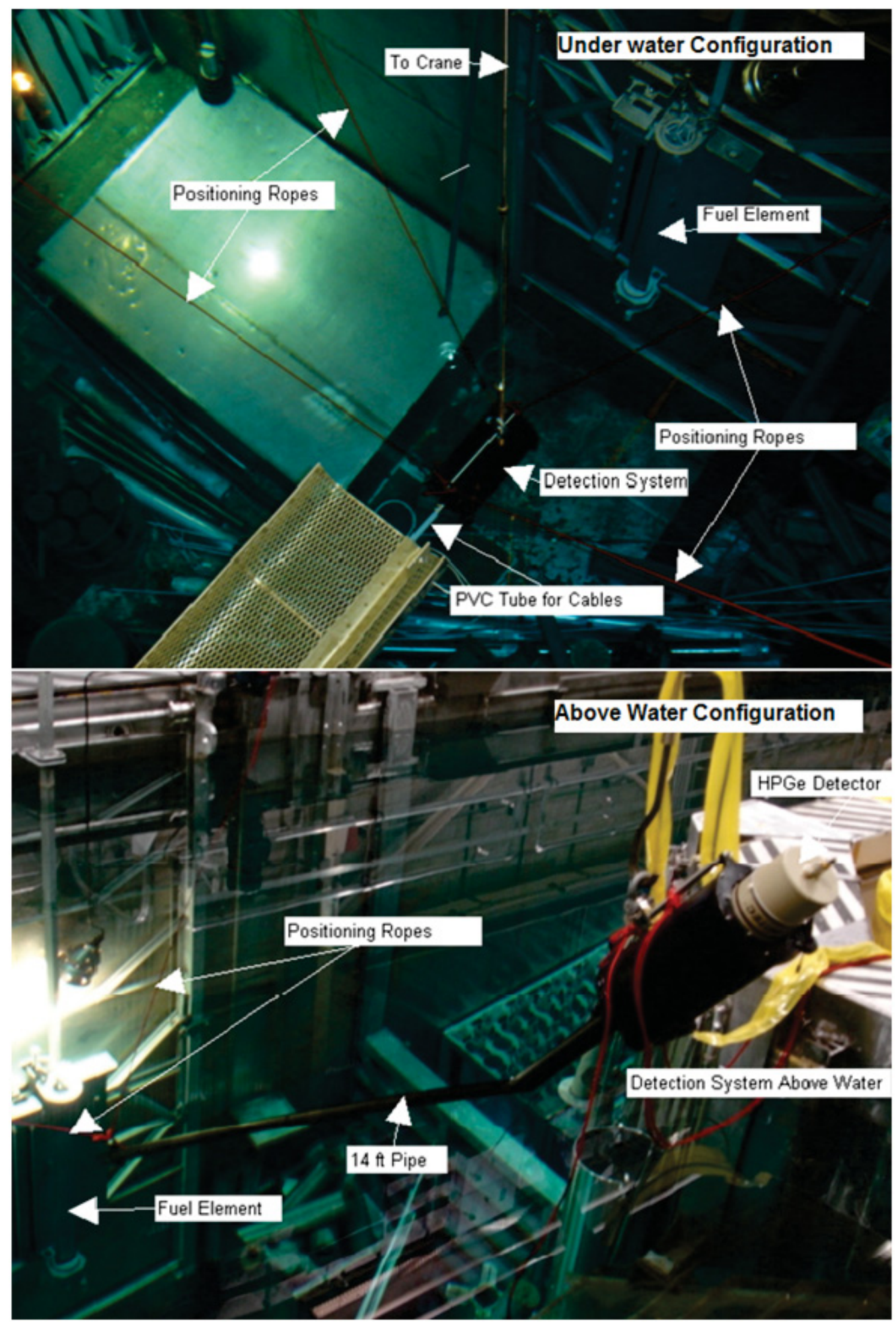

Figure 2.3 This figure shows the measurement configuration for under the water (top) and above the water (bottom) 
Table 2.2 Elements measured during the study along with their initial and final ${ }^{235} \mathrm{U}$ content. Also the Average power for each reactor cycle that the element was exposed.

\begin{tabular}{|c|c|c|c|c|c|c|}
\hline $\begin{array}{l}\text { Fuel } \\
\text { Element } \\
\text { I.D. }\end{array}$ & $\begin{array}{l}\text { No.Cycles } \\
\text { in the } \\
\text { Reactor }\end{array}$ & $\begin{array}{l}\text { Initial } \\
{ }^{235} \mathrm{U} \\
\text { Content } \\
\text { (grams) }\end{array}$ & $\begin{array}{l}\text { Content of } \\
{ }^{235} \mathrm{U} \text { at Time } \\
\text { of } \\
\text { Measurement } \\
\text { (grams) }\end{array}$ & $\begin{array}{l}\text { Average } \\
\text { Element } \\
\text { Power } \\
\text { Cycle } 1 \\
(\mathrm{MW})\end{array}$ & $\begin{array}{l}\text { Average } \\
\text { Element } \\
\text { Power } \\
\text { Cycle } 2 \\
(\mathrm{MW}) \\
\end{array}$ & $\begin{array}{l}\text { Average } \\
\text { Element } \\
\text { Power } \\
\text { Cycle } 3 \\
(\mathrm{MW})\end{array}$ \\
\hline XA652T & 1 & 1073 & 929 & 2.250 & - & - \\
\hline XA379T & 2 & 1073 & 877 & 2.873 & 2.874 & - \\
\hline XA374T & 3 & 1073 & 628 & 2.253 & 2.910 & 2.721 \\
\hline XA826T & 1 & 1073 & 827 & 3.218 & - & - \\
\hline XA815T & 1 & 1073 & 850 & 3.250 & - & - \\
\hline XA377T & 3 & 1073 & 657 & 2.253 & 2.874 & 2.721 \\
\hline XA569T & 1 & 1073 & 866 & 3.125 & - & - \\
\hline XA794T & 1 & 1073 & 804 & 3.130 & - & - \\
\hline XA383T & 2 & 1073 & 754 & 2.253 & 2.910 & - \\
\hline XA638T & 2 & 1073 & 805 & 2.952 & 2.251 & - \\
\hline XA665T & 3 & 1073 & 631 & 2.874 & 2.252 & 2.880 \\
\hline XA573T & 3 & 1073 & 583 & 7.076 & 2.982 & 3.166 \\
\hline XA716T & 2 & 1073 & 777 & 2.353 & 2.256 & - \\
\hline XA796T & 1 & 1073 & 867 & 3.126 & - & - \\
\hline
\end{tabular}




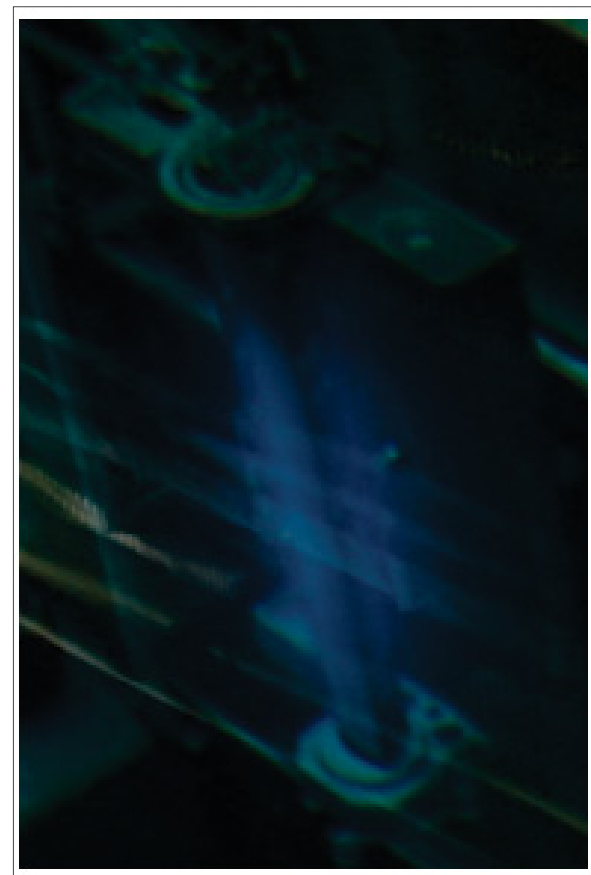

\section{Plate Number 19}

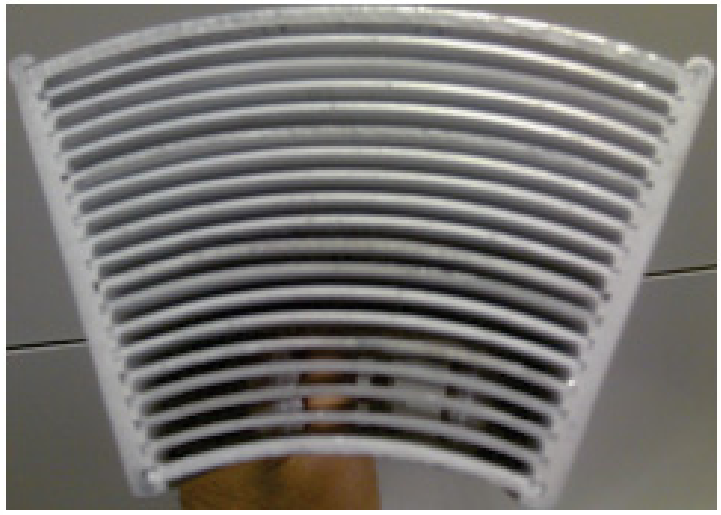

\section{Plate number 1}

Figure 2.4 ATR reactor fuel assembly placed in the turntable (shown on the left). It is $4 \mathrm{ft}$ long and consisted of 19 plates (shown on the right)

Table 2.3 Gamma-ray peaks used for calibrations

\begin{tabular}{|c|c|c|}
\hline Isotope & Half-life & $\begin{array}{c}\text { Gamma-ray peak used } \\
\text { for calibration (keV) }\end{array}$ \\
\hline${ }^{95} \mathrm{Zr}$ & $64.02 \mathrm{~d}$ & 756.7 \\
\hline${ }^{95} \mathrm{Nb}$ & $34.99 \mathrm{~d}$ & 765.8 \\
\hline${ }^{134} \mathrm{Cs}$ & $2.06 \mathrm{yr}$ & 604.7 \\
\hline${ }^{137} \mathrm{Cs}$ & $30.07 \mathrm{yr}$ & 661.6 \\
\hline${ }^{144} \mathrm{Ce}$ & $284.5 \mathrm{~d}$ & 2185.6 \\
\hline
\end{tabular}




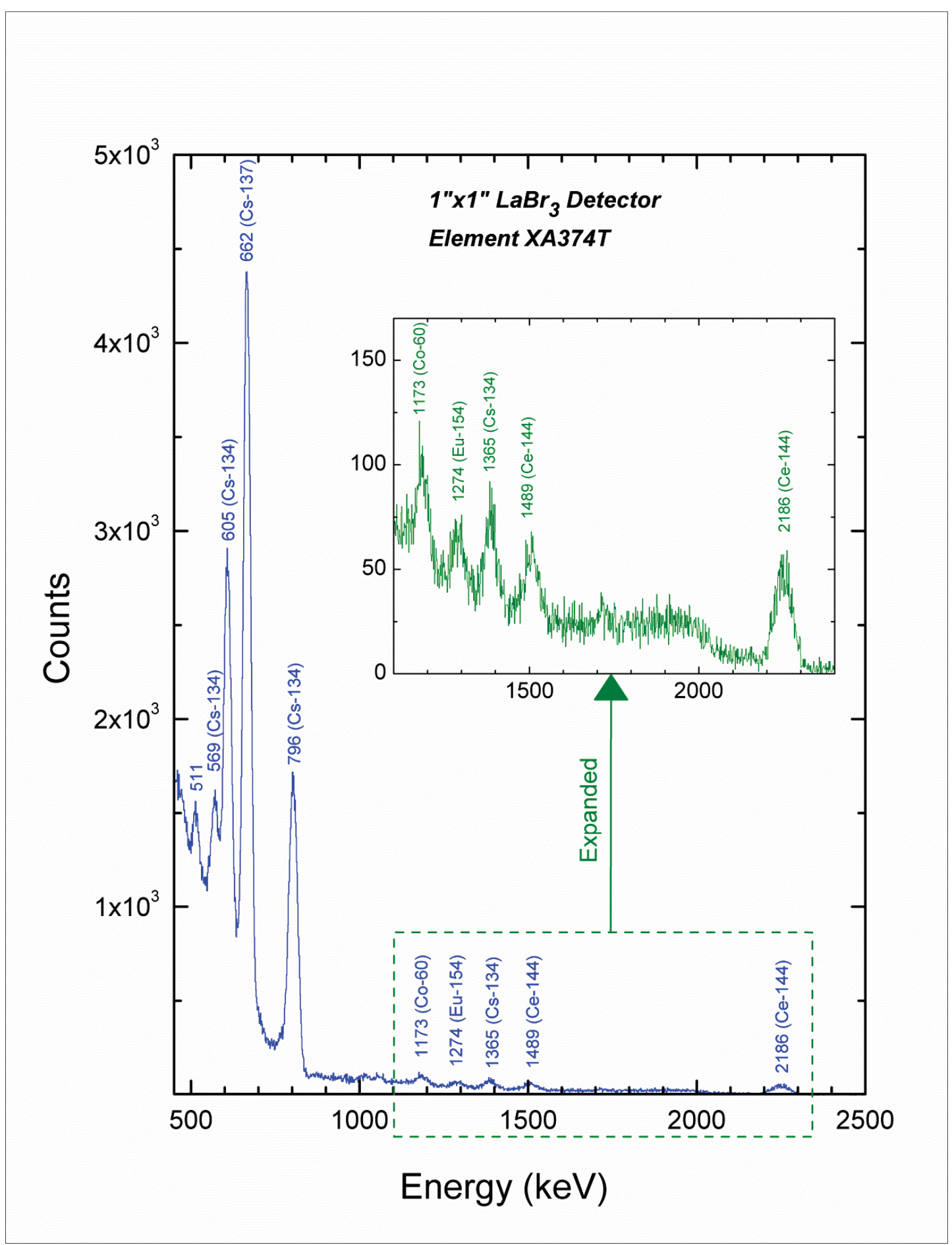

Figure 2.5 Spectrum taken with $\mathrm{LaBr}_{3}$ detector above the water 


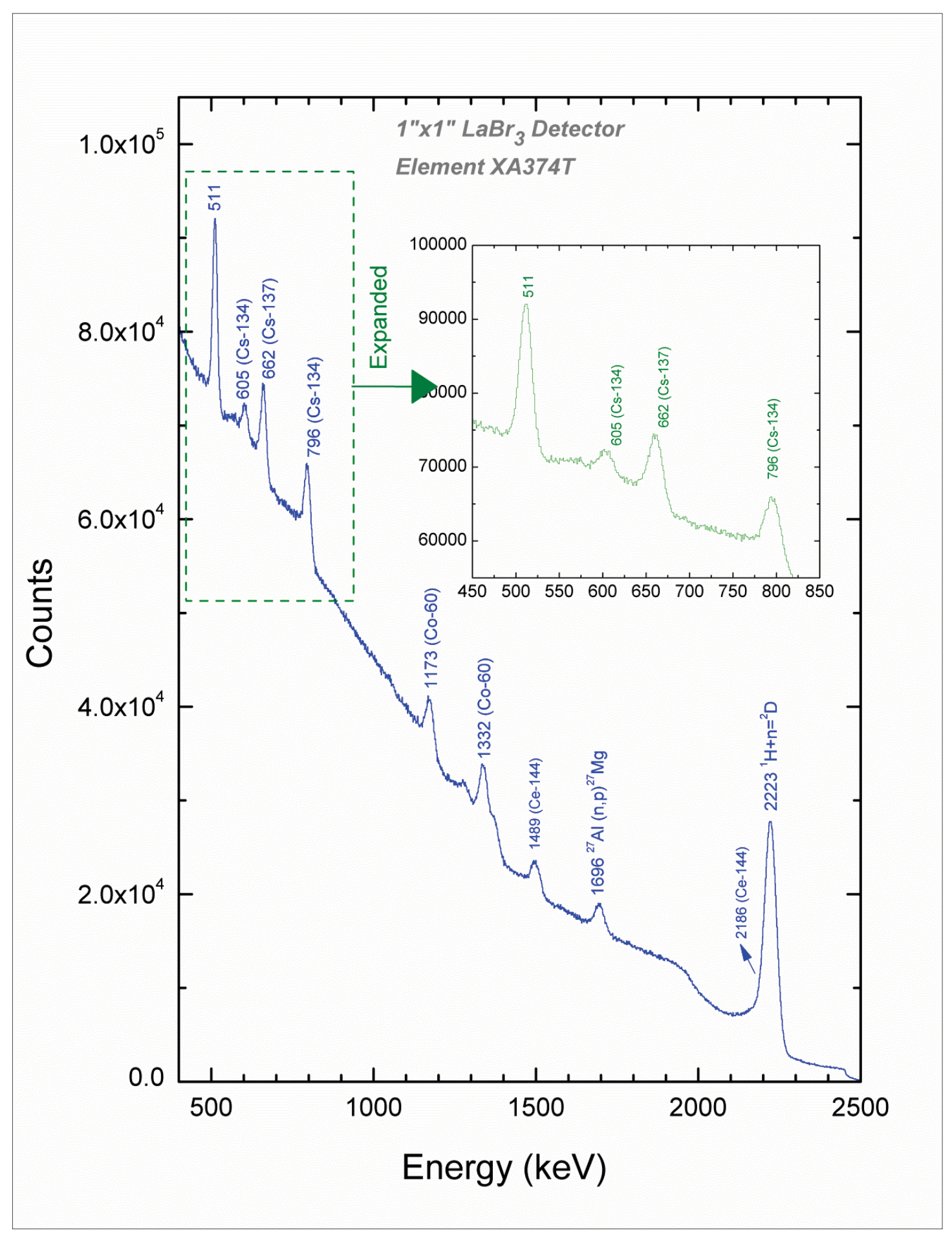

Figure 2.6 Spectrum taken with $\mathrm{LaBr}_{3}$ detector underwater 


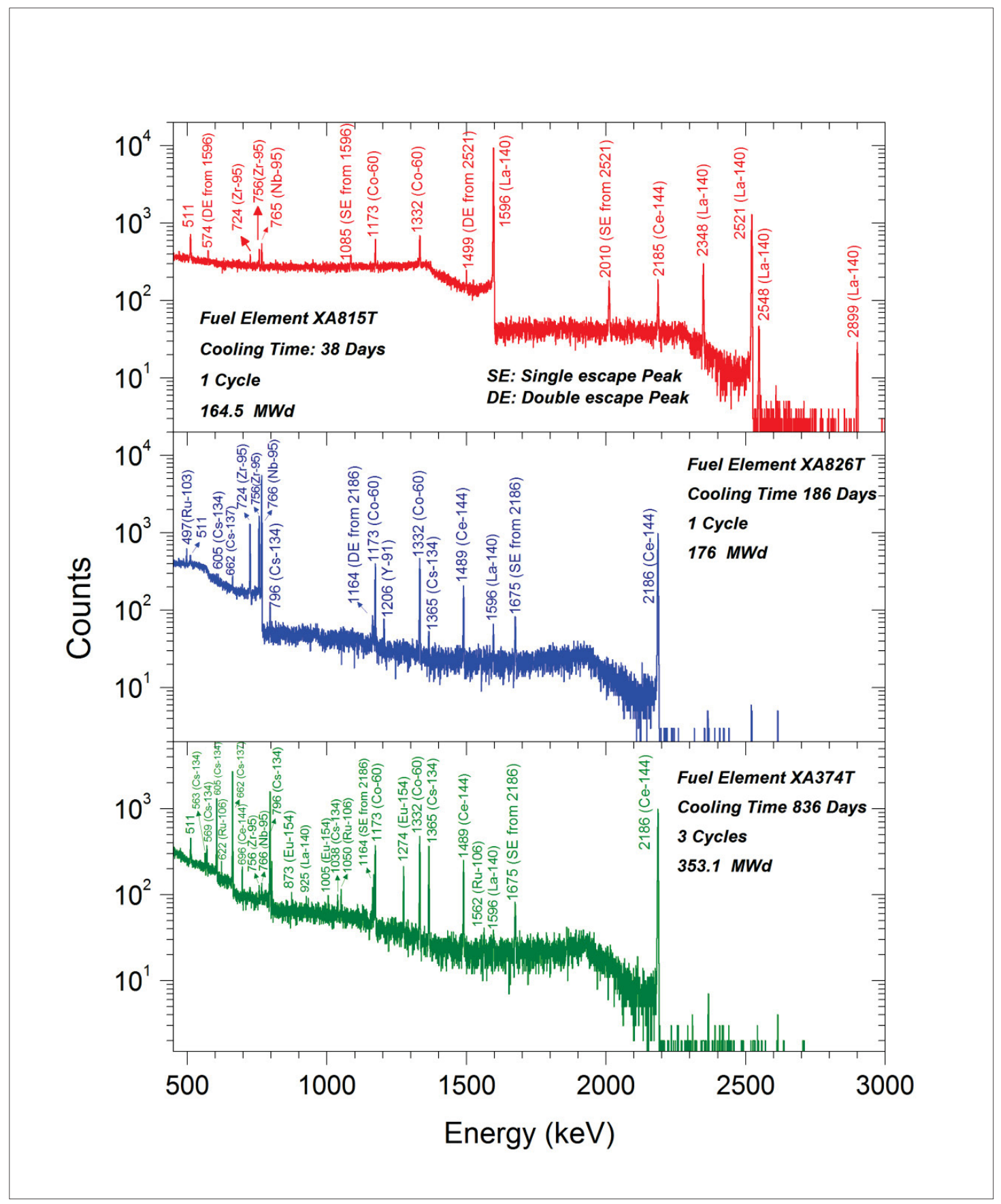

Figure 2.7 Spectra taken with the HPGe detector 


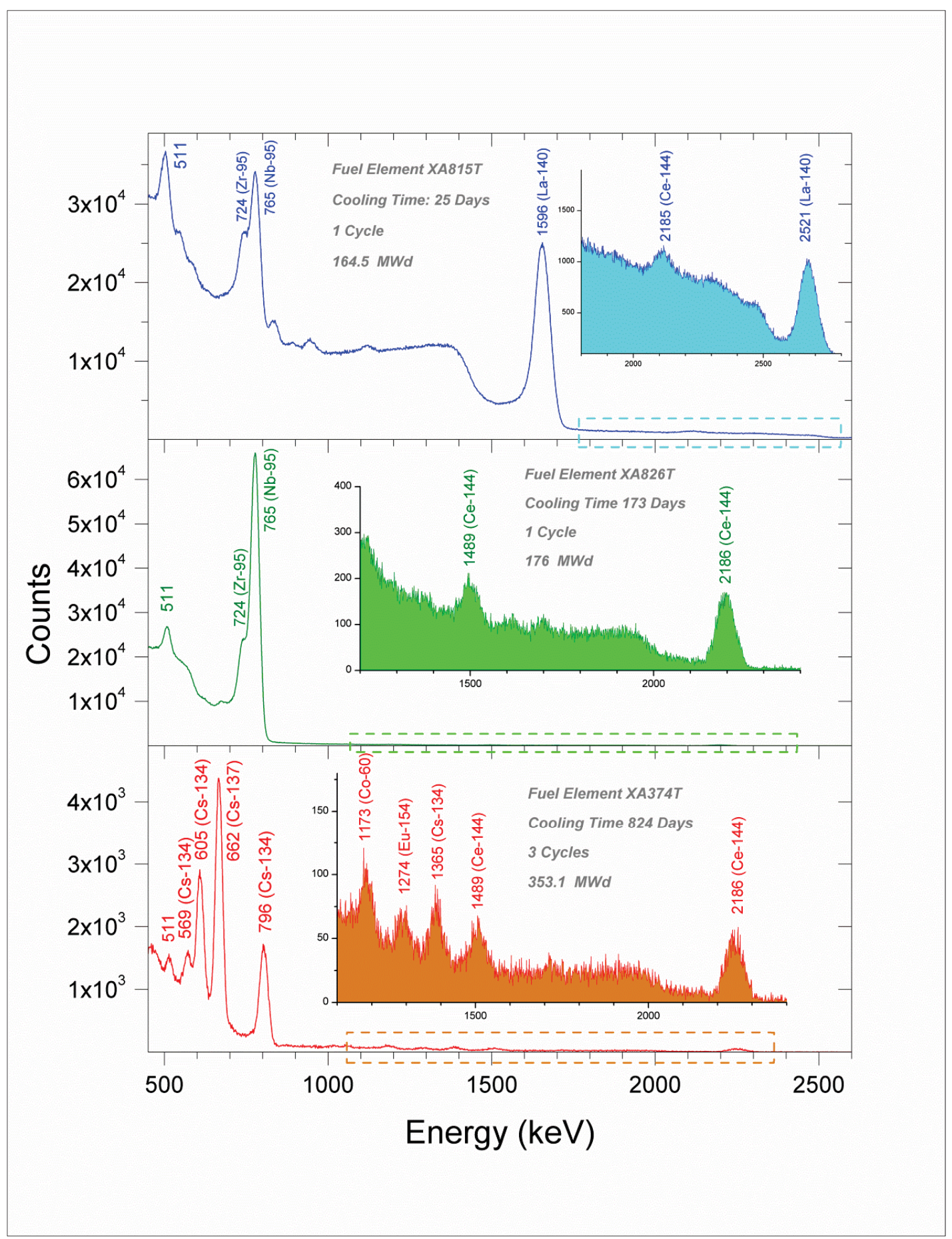

Figure 2.8 Spectra taken with the $\mathrm{LaBr}_{3}$ detector 


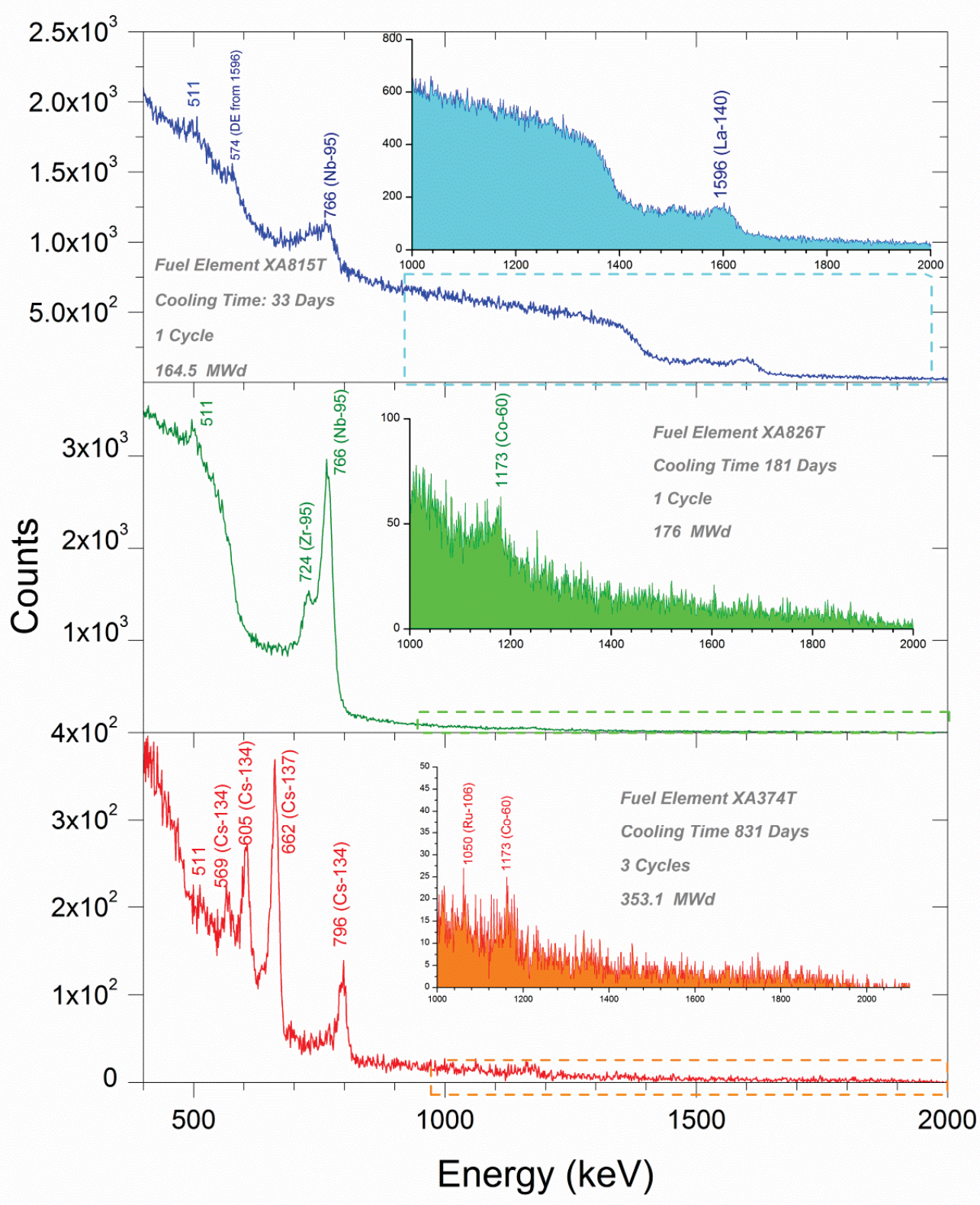

Figure 2.9 Spectra taken with the HPXe detector 


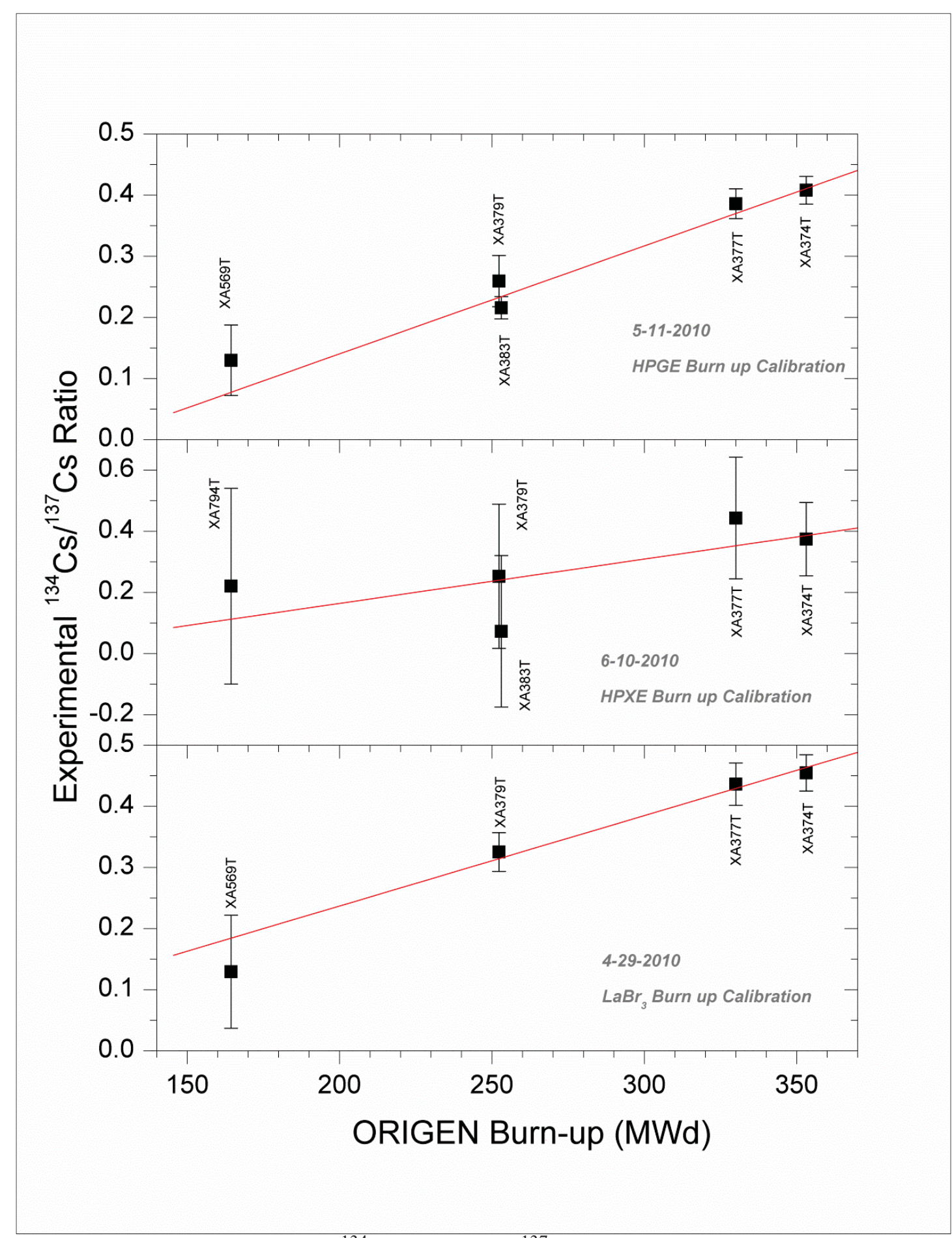

Figure 2.10 Burnup calibration ${ }^{134} \mathrm{Cs}(605 \mathrm{keV}){ }^{137} \mathrm{Cs}(662 \mathrm{keV})$ area ratio using different detectors 


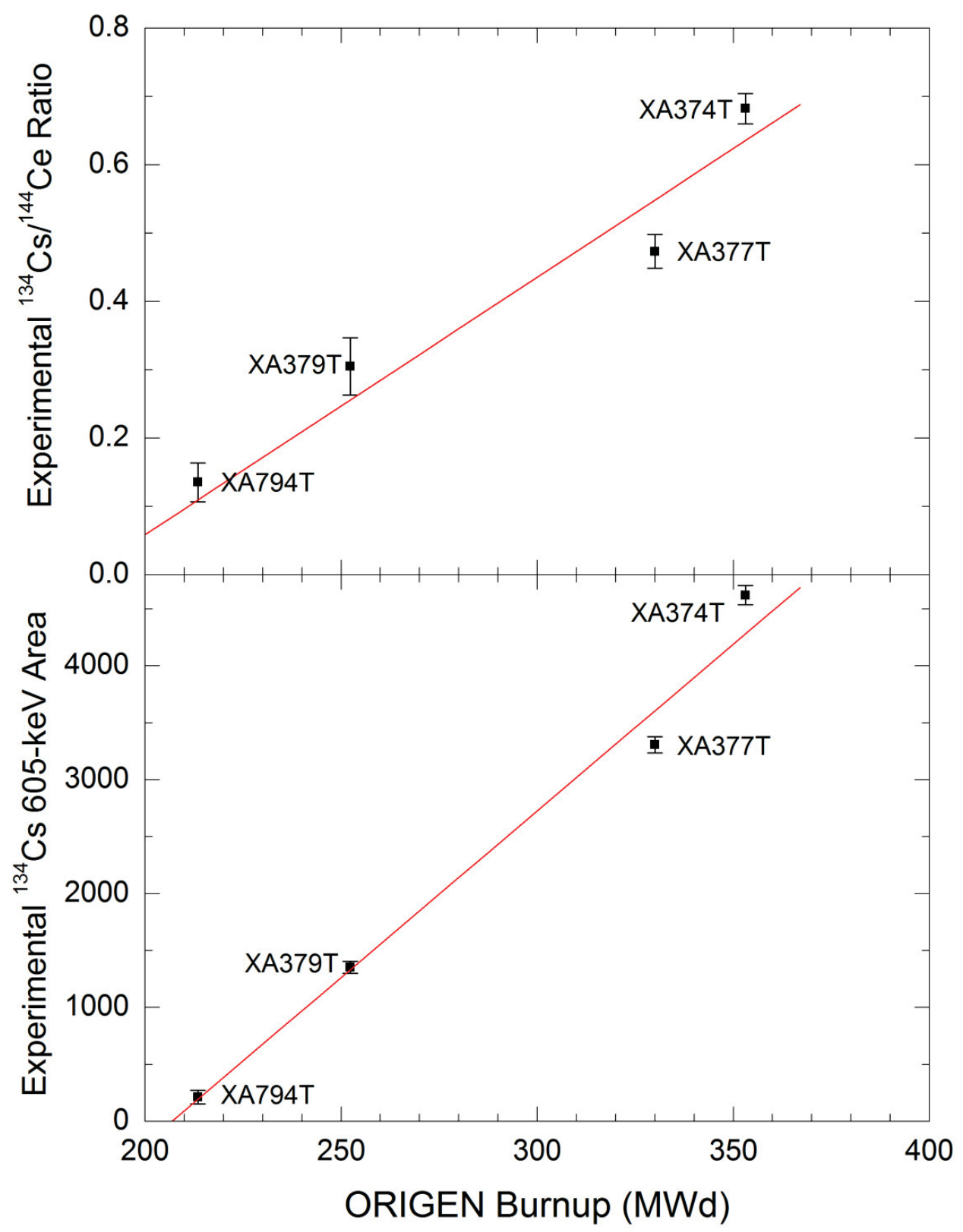

Figure 2.11 Burnup calibration ${ }^{134} \mathrm{Cs}(605 \mathrm{keV}) /{ }^{144} \mathrm{Ce}(2185 \mathrm{keV})$ area ratio and ${ }^{134} \mathrm{Cs}$ $(605 \mathrm{keV})$ area using an HPGe detector 


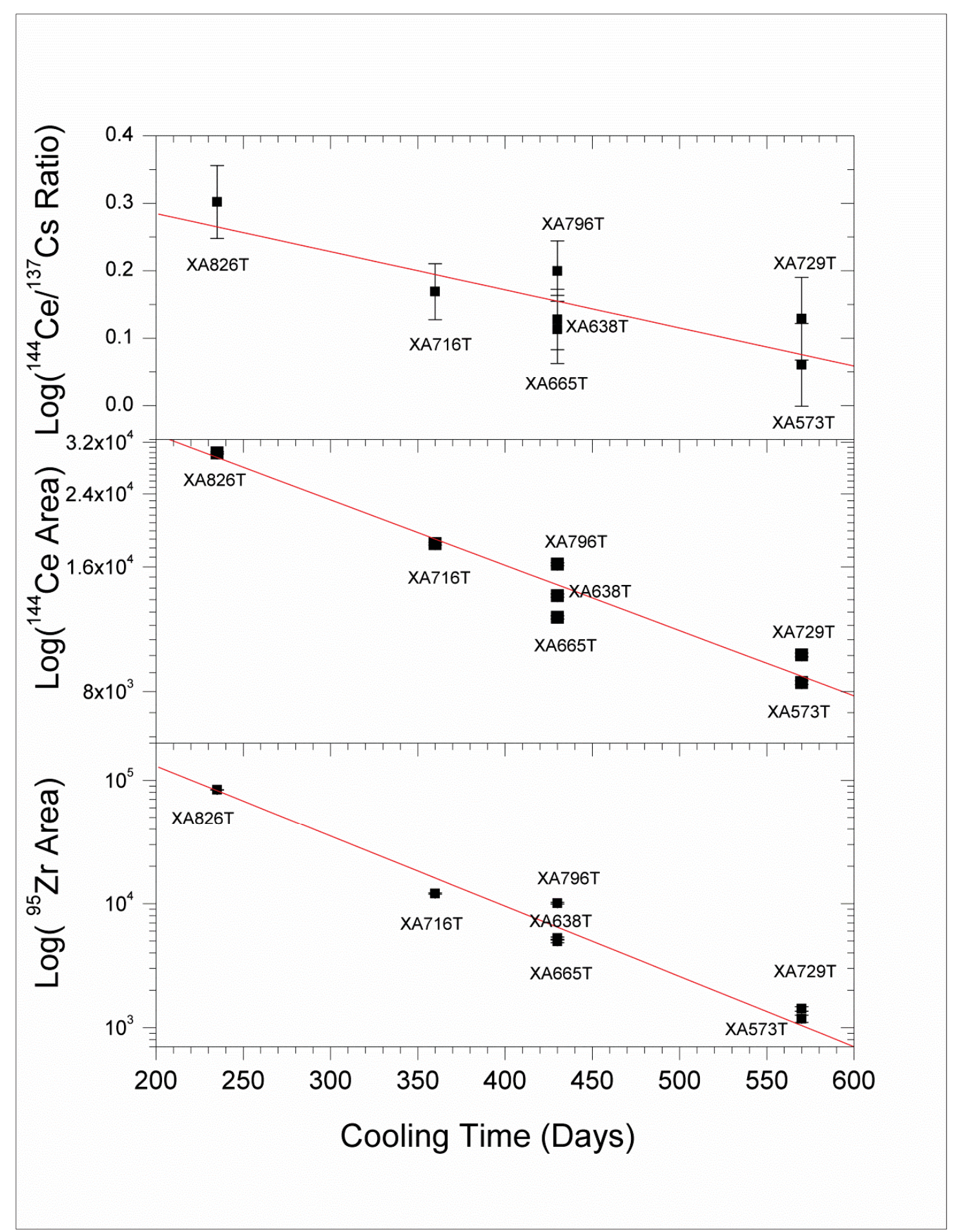

Figure 2.12 Cooling time calibration for different net areas and area ratio using an HPGe detector. The gamma-rays used are $662 \mathrm{keV}$ for ${ }^{137} \mathrm{Cs},{ }^{2185} \mathrm{keV}$ for ${ }^{144} \mathrm{Ce}$, and $756 \mathrm{keV}$ for ${ }^{95} \mathrm{Zr}$. 


\section{$\underline{2.9 \text { References }}$}

1 Reilly, D.; Ensslin, N.; Smith, H.; Kreiner, S. Passive Nondestructive Assay of Nuclear Materials; NUREG/CR-5550 (LA-UR-90-732), Los Alamos N.M., 1991.

2 Ramalho, A. J. G.; Payne, W. E. Spent Fuel Measurements using High Resolution Gamma Systems. Nuclear Materials Management, 1979, 76-82.

3 Phillips ,J. R.; et al., Application of Nondestructive Gamma-ray and Neutron Techniques for the Safeguarding of Irradiated Fuel Materials; LA-8212, Los Alamos N.M., 1980.

4 Phillips , J. R., Irradiated Fuel Measurement. In Passive Nondestructive Assay of Nuclear Materials, Reilley, D.; Ensslin ,N; Smith H.,Ed.; Los Alamos N.M., 1991; Chapter 18, p 529-562.

5 Lebrun, A.; Bignan, G. Nondestructive Assay of Nuclear Low-Enriched Uranium Spent Fuels for Burnup Credit. Nuclear Technology, 2001, 135, 216-228.

6 Terremoto, L.A.; Zeituni, C.A.; Perrotta, J.A.; Da Silva, J.E. Gamma-ray spectroscopy on irradiated MTR fuel elements, Nucl. Instr. and Meth. 2000, 450, 495-514.

7 Oeda, K.; Hirofumi,H.; Hirota, M. ; Natsume, K.; Kumanomido, H. Calibration of Burnup Monitor Installed in Rokkaso Reprocessing Plant. Jounal of Nuclear Science and Technology, 2000, Vol. 37, No. 6,543-547.

8 Pereda, C.B.; Henríquez, C.A.; Klein, J.D.; Medel, J.R. "Burn up Measurements of LEU Fuel for short cooling Times. J. Phys.: Conf. Ser 2008,134.

9 Burra, T.L.; Charltonb,W.S.; Nakhleh, C.W. Assessing confidence in inferring reactor type and fuel burnup: A Markov chain Monte Carlo approach, Nucl. Instr. and Meth. $A$, 2005, Vol. 555, Issues 1-2, pp. 426-434.

10 Matsson, I.; Grapengiesser, B.; Andersson, B. LOKET - a gamma-ray spectroscopy system for in-pool measurements of thermal power distribution in irradiated nuclear fuel, Nucl. Instr. and Meth. A, 2006, Vol. 569, Issue 3, 872-882.

11 Croff, A.J. ORIGEN2 -- A Revised and Updated Version of the Oak Ridge Isotope Generation and Depletion Code; ORNL-5621; 1980.

12 Park, K. J.; et al. Determination of Burnup and PU/U Ratio of PWR Spent Fuels by Gamma-Ray Spectrometry. Nuclear Engineering and Technology, 2009, Vol 41, No.10, 1307-1314. 
13 Bell,M.J.; The ORNL Isotope Generation and Depletion Code; Oak ridge, TN, 1973; ORNL-462.

14 PFEIFER, C. J.; PDQ-7 Reference Manual II;WAPD-TM-947(L); 1971.

15 Tortorelli, J.P.; Housley, K.D. A User's Manual for INEEL Spex Spectral Analysis Software; INEEL/EXT-2001-1435; Idaho Falls, ID, 2001.

16 Nigg, D. W; et al. Advanced Test Reactor Core Modeling Update Project Annual Report for Fiscal Year 2010; INL/EXT-10-19940; Idaho Falls, ID, 2010.

17 Advaced Test Reactor National Scientific User Facility User's Guide; INL/EXT-0814709 ; Idaho Falls, ID, 2009. 


\section{CHAPTER 3}

\section{GAMMA-RAY DETECTOR SELECTION}

The first stage of the study demonstrated that it was possible to obtaining a meaningful gamma signal from fuel elements at the ATR canal and that it was feasible to predict burnup and cooling time using three different kinds of detectors. The first stage of the study also showed that the three detectors can be used to collect data at the ATR canal with different outcomes, but that the HPGe yielded superior results. However, the use of an HPGe detector for canal operations is far from ideal. In a laboratory setting, the HPGe detector will be the best choice; however, the detector is not well-suited for the ATR canal were the environment is harsh and the maintenance of the detector will not always be the primary concern for canal operators. The detector selected for everyday operations for the fuel scanning system has to be able to endure the everyday harsh operation conditions at the ATR fuel canal. From the two detectors used in the study besides the HPGe, the $\mathrm{LaBr}_{3}$ quality data collected and calibration curves performance was superior to the HPXe. Given the fragility of the HPGE and the performance of the rest of the detectors used in the study, it was determined that exploring the possibility of using the $\mathrm{LaBr}_{3}$ detector as an everyday detector for fuel scanning had to be investigated.

Before determining if the $\mathrm{LaBr}_{3}$ detector could be used as a primary detector in the scanning system it was determined that an optimization study to improve the 
resolution of the detector had to be performed, because the everyday detector has to be able to extract the most information out of the fuel elements. The $\mathrm{LaBr}_{3}$ scintillators are reliable in-situ detectors and it was established in Chapter 2 of this thesis that they provide good quality spectra as well as confident ATR burnup prediction calibrations; however, resolution of the spectra collected was inferior to the HPGe detectors. The inferior resolution of the $\mathrm{LaBr}_{3}$ spectra affected the identification of isotopes and increased the error in burnup calibrations. Consequently, in order to explore the possibility of enhancing the resolution of the spectra collected using $\mathrm{LaBr}_{3}$, it was determined that a deconvolution method had to be developed. The results of this study will determine if deconvolution can increase the performance of $\mathrm{LaBr}_{3}$ scintillators for ATR applications and will be a better option to design a more rugged permanent system that can withstand daily operations at the ATR canal.

Enhancement spectra methods have been proven to be successful when applied to lower resolution scintillator detectors ${ }^{18,}$ 19; however, not a lot of research had been performed on the spectrum enhancement of $\mathrm{LaBr}_{3}$ detectors. Chapter 4 presents the worked performed to develop a deconvolution method for a 1 " x 1 " $\mathrm{LaBr}_{3}$ scintillator in order to increase the reliability of this scintillator as a primary option for the permanent system. The first step in developing a spectra deconvolution technique is to calculate the response function of the detector. There are several methods of calculating response functions of detector. The methods can be experimental or computational ${ }^{20,21}$. The approach selected for this study was to determine the response function by Monte Carlo Simulations method ${ }^{22}$; this method can be time-consuming; however, it can also be very effective. Once the response function of the detector has been determined, the next step 
of the process will be to determine which enhancement technique will provide the best results. There are several methods to perform spectra deconvolution ${ }^{23}$; however, the Maximum- Likelihood Expectation Maximization Algorithm (MLEM) ${ }^{24}$ has proven to be very effective. The MLEM algorithm is widely used in medical imaging and had been successfully applied in the past to lower resolution scintillators. After the response function of the detector was calculated and the MLEM was implemented in Matlab, simulated data were created in order to test the performance of the method. The model data created were one-isotope and simulated ATR fuel data. The one-isotope data were used to calculate performance parameters of the convoluted and deconvoluted data while the fuel data were simulated to investigate the performance of burnup calcibration curves before and after deconvolution. 


\title{
CHAPTER 4
}

\author{
GAMMA-RAY SIMULATED SPECTRA DECONVOLUTION \\ OF A $\mathrm{LaBr}_{3} 1$ 1"X1" SCINTILLATOR FOR \\ NONDESTRUCTIVE ATR FUEL \\ BURNUP ON-SITE \\ PREDICTIONS
}

\begin{abstract}
$\underline{4.1 \text { Abstract }}$
A deconvolution method for a $\mathrm{LaBr}_{3} 1$ "x1" detector for nondestructive Advanced Test Reactor (ATR) fuel burnup applications was developed. The method consisted of obtaining the detector response function, applying a deconvolution algorithm to 1 "x 1 " $\mathrm{LaBr}_{3}$ simulated data, along with evaluating the effects that deconvolution have on nondestructively determining ATR fuel burnup. The simulated response function of the detector was obtained using $\mathrm{MCNPX}^{10}$ as well with experimental data. The MaximumLikelihood Expectation Maximization (MLEM) deconvolution algorithm was selected to enhance one-isotope source-simulated and fuel-simulated spectra. The final evaluation of the method consisted of measuring the performance of the fuel burnup calibration curve for the convoluted and deconvoluted cases.

The methodology was developed in order to help design a reliable, high resolution, rugged, and robust detection system for the ATR fuel canal capable of
\end{abstract}


collecting high performance data for model validation, along with a system that can calculate burnup using experimental scintillator detector data.

\section{$\underline{4.2 \text { Introduction }}$}

The design of a permanent fuel scanning system to nondestructively determine burnup and cooling time of Advanced Test Reactor (ATR) fuel is underway at the Idaho National Laboratory (INL). The final design of the ATR fuel permanent scanning apparatus requires a gamma detector capable of collecting high-quality spectra needed for model validation and on-site burnup calibration predictions in a high background radiation environment. The gamma spectroscopy data are needed for validation of the new suite of modeling tools that are being developed and will be implemented at the ATR to support canal operations. The data will also be used for burnup prediction of fuel elements in order to provide reliable on-site information to the ATR fuel management team. The first stage of the study was completed and it consisted of determining the feasibility of implementing a fuel scanning system at the ATR canal ${ }^{(6)}$. The study established a protocol to collect meaningful gamma spectral data and a method to predict fuel burnup and cooling time on-site for ATR fuel elements at the storage canal. Due to the in-situ nature of the measurements, and the variability and pace of canal operations, it was necessary to design a system that can withstand the daily ATR working environment, which includes having a rugged compact gamma spectroscopy detector that is capable of providing high-quality spectra without constant maintenance. The determination of which detector is better suited for the final fuel scanning permanent system cannot be made 
based solely on the quality of the spectra, but the decision had also to take into account the fact that the detection system will be placed at the ATR canal.

The fuel scanning permanent system feasibility study used three different detectors (HPGe, HPXe, and $\mathrm{LaBr}_{3}$ ) for ATR canal in-situ measurements. The three detectors tested were capable of retrieving a signal at the ATR fuel canal; however, the fuel burnup predictions and quality of the data varied from one detector to the other ${ }^{(6)}$. The preliminary results obtained showed as expected that the HPGe detector gives the best quality spectra from among the three detectors used in the study. However, the need for HPGe detectors to be operated at cryogenic temperatures, their overall size, and fragility limit their range of applications and complicate their implementation for in-situ measurements of spent fuel elements at the ATR canal ${ }^{6}$. Also, HPGe detectors cannot be used in hostile environments such as in a high radiation field without adequate shielding. The HPXe detectors are excellent in-situ detectors; however, during the study, it was determined that they could not be consider a primary option for the permanent fuel scanning system because of the quality of the spectra, high error calibration curves, and the long counting time needed to obtain meaningful spectra. However, they are still being considered as an alternative detector in the final design of the permanent system to measure fuels with very short cooling times (0-20 days). During the study, it was also determined that because of their properties, prediction results, and performance, the $\mathrm{LaBr}_{3}$ scintillators should be consider as primary alternative for the final design of the permanent system. The $\mathrm{LaBr}_{3}$ detectors performed very well under harsh conditions and needed little maintenance, making them ideal for the ATR canal operations. In addition, the $\mathrm{LaBr}_{3}$ scintillator gamma spectra obtained at the ATR canal were also used to predict 
ATR fuel burnup; however, there was a higher error associated with the $\mathrm{LaBr}_{3}$ burnup calibration curves when compared to curves obtained using HPGe spectra ${ }^{(6)}$. Finally, it was concluded that in order to improve the range and quality of the burnup predictions obtained with the $\mathrm{LaBr}_{3}$ scintillator data, a deconvolution process had to be developed and tested.

The development of the deconvolution process for burnup and cooling time applications started by estimating the response function of the $\mathrm{LaBr}_{3} 1$ "x 1 " detector using the Monte Carlo simulations. There are several methods to calculate the response functions of a detector. The methods can be experimental or computational ${ }^{3,4}$. The approach selected for this study was to determine the response function using the Monte Carlo simulation method ${ }^{5}$. This method can be time-consuming; however, it is very effective. Once the detector response function was determined, an MLEM algorithm was used to enhance the energy of the $\mathrm{LaBr}_{3}$ for single isotope and fuel simulated spectra. The last stage of the study involves comparing the performance of the convoluted and deconvoluted burnup calibration curves.

\section{$\underline{4.3 \mathrm{LaBr}_{3}} \underline{1 \text { "x } 1 \text { " Scintillator Energy Response Function Determination }}$}

The first step in developing an enhancement technique for the permanent system is to determine the detector response function. Successful implementation of spectrum deconvolution process depends on accurately predicting the detector response as a function of incident gamma-ray energy. The detector response function can be obtained either experimentally or computationally; both methods are time-consuming. The main drawback of the experimental technique is the need for numerous single peak energy 
sources and the time that it will take to obtain meaningful counts. The modeling path has three possible options for computing the response function: numerical interpolation, derivation of an empirical analytical function from available experimental response functions from and Monte Carlo simulations ${ }^{1}$. For this study, the Monte Carlo simulation route was chosen. This route is time-consuming; however, it is the method that can provide better accuracy and control of the energy ranges. The simulation time can be reduced by using a computer cluster. The process to obtain the response function of the $1 " x 1 " \mathrm{LaBr}_{3}$ consisted in:

$>$ Designing an experiment.

Performing measurements for several gamma radiation sources.

Calculating the Gaussian Energy Broadening parameters needed in MCNPX.

$>$ Simulating the experimental setup in MCNPX and validating the model with experimental data collected.

\section{$\underline{4.4 \text { Experimental Measurements }}$}

The determination of the response function for the $\mathrm{LaBr}_{3}$ detector began by setting up a simple geometry experiment; for this study, the detector was placed on a stainless steel table (Fig. 4.1) and the source was placed $25 \mathrm{~cm}$ from the detectors aluminum entrance window.

The detector used for this study's experiments was a BrilLanCe380 Saint-Gobain detector, which consists of a 1"x1" Labr $_{3}$ scintillation crystal coupled to a photomultiplier tube. The photomultiplier and the scintillator crystal are contained in an outside aluminum sealed housing with an aluminum entrance window. Performing experimental 
measurements to collect data had two main purposes; to calculate the broadening parameters needed to give the MCNPX simulations Gaussian distribution energy peaks and to validated the experiment simulation. The counting time of each experimental measurement was 68,400 seconds. Background measurements were taken in between single isotope calibration source measurements in order to obtain the net counts of each source. The radioactive sources used in this study are shown in Table 4.1.

\section{$\underline{4.5}$ Monte Carlo Simulation}

The geometrical model of the $\mathrm{LaBr}_{3}$ detector for the experimental measurements was simulated as two concentric aluminum cylinders. The first cylinder represents the sealed photomultiplier chamber aluminum housing ( $44.5 \mathrm{~cm}$ radius- $114 \mathrm{~cm}$ height). The second cylinder simulates the aluminum housing with an aluminum entrance window (30.4 $\mathrm{cm}$ radius- $26.1 \mathrm{~cm}$ height) containing the $\mathrm{LaBr}_{3}$ scintillator crystal $(25.4 \mathrm{~cm}$ radius$25.4 \mathrm{~cm}$ width). Accurately representing the geometry of the detector was just the first step in simulating a real system. In order to have a model that truly represents the interaction between detectors and particles measured, the pulse height simulation in MCNPX has to be given a more realistic Gaussian energy distribution. MCNPX ${ }^{10}$ contains a special tally option in which experimental data generated parameters can be input to the simulation in order to give the spectra the required Gaussian shape ${ }^{(9)}$. The special tally within MCNPX ${ }^{10}$ is called the Gaussian Energy Broadening (GEB) option The GEB tally gives the detector-simulated data a Gaussian shape by using the unbroadened energy input and calculated spectral data along with user-specified inputs to solve eq. $4.1^{9}$. 


$$
f(E)=C e^{-\left(\frac{E-E o}{A}\right)^{2}}
$$

where $\mathrm{E}=$ broadened energy, $\mathrm{E} \mathrm{O}=$ unbroadened energy of the tally, $\mathrm{C}=$ normalization constant, and $\mathrm{A}=$ Gaussian width, defined by:

$$
A=\frac{F W H M}{2 \sqrt{\ln 2}}
$$

In order to obtain the Gaussian width needed to solve equation 1, the full width at half maximum (FWHM) of real experimental data is indirectly provided by the user by specifying the three parameters (a,b and c) in eq. 4.3 required by the GEB tally

$$
F W H M=a+b \sqrt{E+c e^{2}}
$$

The GEB parameters for the detection system setup were obtained using the energy calibrated FWHM data acquired from experimental measurements of different gamma sources (Table 4.1) and by performing a nonlinear least square analysis using Matlab. Below are the GEB tally parameters calculated using the experimental measurements needed for MCNPX to give the model spectra Gaussian shape peaks:

$$
a=0.0068 \mathrm{MeV} \quad b=0.0058 \mathrm{MeV}^{1 / 2} \quad c=14.9501 \mathrm{MeV}^{-1}
$$

Once the simulated spectra had Gaussian pulse height distribution energy shaped peaks, the next step was to corroborate that the simulation responses calculated had good agreement with the experimental gamma-ray sources measurements. The validation analysis consisted of comparing the simulated gamma-ray sources $\left({ }^{60} \mathrm{Co},{ }^{137} \mathrm{Cs}\right.$, and ${ }^{226} \mathrm{Ra}$ ) spectra against the experimental data collected to determine if there was a good agreement between the two. Figure 4.2 shows the simulation and experimental spectra of the multi-energy source ${ }^{226} \mathrm{Ra}$. Also the plot shows how adding the Gaussian energy broadening to the $\mathrm{MCNPX}^{10}$ pulse height broadening tally allows for a more accurate 
representation of the physics of the system. The plot shows that the MCNPX pulse height data peaks were broadened to match the experimental data. The plot also shows that there are some discrepancies in the areas below the peaks; however, for this study, because the protocol will only be applied to simulated data, the interest was only in obtaining GEB parameters that will give the simulation a realistic Gaussian peak shape, not in an absolute match with the experimental data.

\subsection{Response Function Calculation}

After the GEB parameters were calculated using experimental measurements, the pulse height data distribution for various energy intervals for the 1 "x1" $\mathrm{LaBr}_{3}$ detector was calculated. The determination of the response function matrix based on experimental measurements was done by modeling 1301 mono-energetic point sources located at 25 $\mathrm{cm}$ from the detector tallying surface. The mono-energetic energy sources varied from 0 to $2.5 \mathrm{keV}$ with a resolution of $0.5 \mathrm{keV}$. Each mono-energetic $\mathrm{MCNPX}^{10}$ simulation was done using $1 \times 10^{10}$ particles. The calculations were performed using the ICESTORM computer cluster at the Idaho National Laboratory; each MCNPX ${ }^{10}$ simulation was executed using 32 processors.

\section{$\underline{4.7 \text { Spectra Deconvolution }}$}

Spectra deconvolution (or spectra enhancement) is a technique that has the objective of obtaining the most information out of a spectrum taken with a detector measurement system. The theory behind this technique is that especially in scintillation detectors the photon incident on the detector crystal may lose part of its energy by 
photoelectric absorption, Compton scattering, or pair production ${ }^{(8)}$; however, this information is still recorded in the spectra, it is just not observed in the appropriate energy region. The deconvolution technique improves the energy resolution of spectra by moving the counts from undesired regions to the appropriate photo-peaks ${ }^{8}$.

Deconvolution is a powerful technique and when done correctly, it enhances the resolution of spectra, allowing for poor resolution detectors to perform tasks that otherwise would only be suitable for high resolution detectors. The unfolding process is also very time-consuming and prone to errors. The deconvolution process is highly dependent on the accuracy of the response function. In addition, the response function calculations are extremely geometry-dependant so every time that a different configuration is introduced, a new response function has to be recalculated.

The deconvolution of a gamma spectrum can be described in a general manner without reference to any particular experimental system ${ }^{8}$. The problem can be posed using an equation (eq. 4.4) that describes the physical process of recording the pulse height data distribution by a detector as the convolution of the true gamma-ray spectrum emitted by a source and the response function of the detector

$$
M(E)=\int_{0}^{\infty} R\left(E, E_{o}\right) S(E)
$$

where $M(E)$ is the measured spectrum, and $R\left(E, E_{o}\right)$ is the pulse height data distribution for various energy intervals (response function), and $S(E)$ is the incident spectrum (actual gamma ray spectrum emitted by a radioactive source). 


\section{$\underline{4.8 \mathrm{LaBr}_{3}} \underline{\text { Spectra Deconvolution of Single Isotopic and Fuel }}$}

\section{Simulated Sources using the Maximum Likelihood}

\section{Expectation Maximization Algorithm}

The integral representing (eq. 4.4) the spectrum collection can be discretize and represented as a matrix in order to be solved numerically:

$$
\begin{aligned}
& M=R S
\end{aligned}
$$

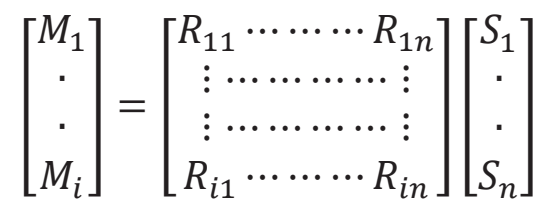

where $M_{i}$ represents the true detector counts, $R_{i}$ is the response function of the detector, and $S_{i}$ represents the discretized actual spectrum. Ideally, (eq. 4.5) can be solved by a simple matrix inversion.

$$
S=R^{-1} M
$$

However, the response function matrix for most nuclear applications is a sparse matrix. Specifically, for the problem discussed in this paper, it was quickly determined that the direct inverse matrix method (maximum likelihood method) was not appropriate for this study, because the response function is a lower triangular matrix (zeros in the top diagonal part of the matrix). The solution to eq. 4.5 can be found using least square fitting iterative algorithms such as GRV_FC33 and SAND-II ${ }^{13}$ as well as using the probabilistic maximum entropy method. For this study, the Maximum Likelihood Fitting by Expectation Maximization (MLEM) was chosen for the reason that it had previously shown success deconvoluting low resolution scintillator spectra ${ }^{1}$. 
The maximum likelihood fitting by expectation (MLEM) is an iterative algorithm (11) designed to obtain the best estimate of the true measured spectrum (incident spectrum). The algorithm starts by creating an approximation of the system matrix which includes an estimate of the measured incident spectrum $\left(\mathrm{x}_{\mathrm{i}}\right)$ for a particular set of set detector counts $\left(\mathrm{y}_{\mathrm{i}}\right)^{7}$.

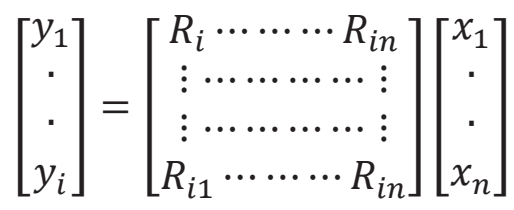

This can be expressed in summation form as

$$
y_{i}=\sum_{n=1}^{n} R_{i n} x_{n}
$$

Assuming that the true detector counts $M_{i}$ are also known, and knowing that they follow a Poisson distribution, it can be inferred that the probability of observing a value for $M_{i}$ given an estimated mean value for $y_{i}$ for one energy bin can be represented by:

$$
p\left(M_{i} \mid y_{i}\right)=\frac{e^{-y_{i} y_{i}{ }^{M i}}}{M_{i} !}
$$

In order to expand eq. 4.10 to encompass all the true measured spectra counts $\left(M_{i}\right)$ for a determine set of energy bins and the estimated of incident spectrum counts $\left(\mathrm{x}_{\mathrm{i}}\right)$, a Poisson product distribution is calculated.

$$
L(M \mid x)=\prod_{i=1}^{i} p\left(y_{i} \mid M_{i}\right)=\prod_{i=1}^{i} \frac{e^{-y_{i}} y_{i}^{M i}}{M_{i} !}
$$

where $L(M \mid x)$ represents the likelihood probability that the true measured spectra counts $\left(M_{i}\right)$ is equal to the detector counts $\left(\mathrm{y}_{\mathrm{i}}\right)$. The MLEM algorithm goal is to maximize the likelihood function (eq. 4.10) in order to obtain the detector counts $\left(\mathrm{y}_{\mathrm{i}}\right)$ that closely fits the measured spectra counts $\left(M_{i}\right)$ by finding the "maximum-likelihood" estimate $\left(\mathrm{x}_{\mathrm{i}}\right)$ of 
the actual gamma-ray spectrum counts $\left(S_{i}\right)$. To mathematically simplify the maximization of the likelihood function $\mathrm{L}$ the logarithm of the function is calculated instead.

$$
\begin{aligned}
\ln (L) & =\sum_{i=1}^{i}\left[-y_{i}+M_{i} \ln y_{i}-\ln \left(M_{i} !\right)\right] \\
& =\sum_{i=1}^{i}\left\{-\sum_{n=1}^{n} R_{i m} x_{i}+M_{i} \ln \left[\sum_{n=1}^{n} R_{i n} x_{i}\right]-\ln \left[M_{i} !\right]\right\}
\end{aligned}
$$

Once the logarithm of the likelihood function is calculated, the function can be maximize by taking the derivative of eq. 4.12 with respect to the estimate of incident spectrum counts $\left(\mathrm{x}_{\mathrm{i}}\right)$.

$$
\frac{\partial \operatorname{In}(L)}{\partial x_{k}}=\sum_{i=1}^{i}\left[\frac{\partial \mathrm{y}_{\mathrm{d}}}{\partial x_{k}}+\frac{M_{i}}{y_{i}} \frac{\partial y_{i}}{\partial x_{k}}\right]=0
$$

From eq. 4.9 it is known that $y_{i}=\sum_{n=1}^{n} R_{i n} x_{n}$, so it can be derived that:

$$
\frac{\partial y_{i}}{\partial x_{k}}=\frac{\partial y_{i}}{\partial x_{k}}\left[\sum_{n=1}^{n} R_{i m} x_{n}\right]=R_{j m}
$$

Substituting eq. 4.14 into eq. 4.13 yields

$$
\sum_{i=1}^{i}\left[\frac{M(i)}{y_{i}} R_{j k}-R_{j k}\right]=0
$$

In order to have everything in terms of $\left(\mathrm{x}_{\mathrm{i}}\right)$ and $R_{j m}$ substituting eq. 4.9 into eq. 4.15 gives

$$
\sum_{i=1}^{i} R_{j k}=\sum_{i=1}^{i} \frac{M_{i}}{\sum_{B=1}^{B} R_{i m} x_{i}} R_{j k}
$$


This can be restated as:

$$
\frac{1}{\sum_{d=1}^{D} R_{j m}} \sum_{i=1}^{i} \frac{M_{i}}{\sum_{n=1}^{n} R_{i m} x_{i}} R_{j k}=1
$$

In order to find the best estimate of the incident spectrum counts $\left(\mathrm{x}_{\mathrm{i}}\right)$ while satisfying eq. 4.11, this technique is applied. In this algorithm, it is assumed that $x_{k}^{(n)}$ is the best estimate of the actual gamma-ray spectrum counts $\left(S_{i}\right)$.

$$
x_{k}^{(n+1)}=x_{k}^{(n)}\left\{\frac{1}{\sum_{i=1}^{i} R_{j k}} \sum_{i=1}^{i}\left[\frac{M_{i}}{\sum_{n=1}^{n} R_{i k} x_{i}^{(n)}} R_{j k}\right]\right\}
$$

The algorithm calculates a new value for $x_{i}^{(n+1)}$ in each iteration and it continues until a predetermine tolerance value is reached. The tolerance value is calculated using the mean squared difference between consecutive iterations

$$
S=\sum_{i=1}^{i}\left(x_{i+1}^{(n)}-x_{i}^{(n)}\right)^{2}
$$

\section{$\underline{4.9 \text { One-Isotope Simulated Sources }}$}

The MLEM algorithm described above was implemented in MATLAB and was tested using simulated one-isotope data created within $\mathrm{MCNPX}^{10}$. The data were modeled based on the experimental setup discuss in the previous section; each isotopic source consisted of $1 \times 10^{10}$ counts. The results for one peak source ${ }^{137} \mathrm{Cs}$, double peak source ${ }^{60} \mathrm{Co}$, and multiple peaks source ${ }^{226} \mathrm{Ra}$ are presented in Fig 4.3, 4.4, and 4.5, respectively.

It can be seen from the three istotopic sources $\left({ }^{137} \mathrm{Cs},{ }^{60} \mathrm{Co}\right.$, and $\left.{ }^{226} \mathrm{Ra}\right) \mathrm{MLEM}$ spectra comparison plots that the Compton scattering region along with the backscatter peak counts where successfully moved to the appropriate Gaussian shape photo-peaks. In order to further quantify the efficiency of the MLEM deconvolution, the performance 
parameters peak-to-Compton and peak-to-total ratio in conjunction with the energy resolution for the ${ }^{137} \mathrm{Cs}$ and ${ }^{60} \mathrm{Co}$ simulated sources were calculated. In addition to the

energy resolution for the ${ }^{226} \mathrm{Ra}$, two major photo-peaks were also calculated. The results for all one-isotopic sources parameters measured are presented in Table 4.2.

The numerical results in Table 4.2 confirm that the energy resolution improves greatly for each one of the photo-peaks measured for the three one-isotope sources. The table also shows a significant improvement for the deconvoluted spectra parameters peak-to-Compton and peak-to-total ratios for ${ }^{137} \mathrm{Cs}$ and ${ }^{60} \mathrm{Co}$ simulated sources.

\section{$\underline{4.10 \text { Fuel Simulated Sources Deconvolution }}$}

Once the MLEM deconvolution algorithm was implemented and tested for the one-isotope simulated sources, a fuel surrogate simulated source was created and modeled in $\mathrm{MCNPX}^{10}$. The source modeled was developed because the ATR fuel data collected during the study could not be directly used to calculate the response function of the 1 "x1" $\mathrm{LaBr}_{3}$ detector for the system used and there is a need to test the performance of the MLEM method for burnup applications. The detection system used in the study was a temporary setup positioned using a crane and ropes. The experimental setup used during the study did not have the capability of measuring the distance between system and target (fuel) or the angle of the detection system in regards to the fuel. Consequently, knowing the dimensions of the detection system with any degree of accuracy in order to create an exact $\mathrm{MCNPX}^{10}$ model for the calculation of the detector response function was unfeasible. The response function calculation for deconvolution of spectra using $\mathrm{MCNPX}^{10}$ needs a precise representation of the experimental setup in order for the model 
to accurately simulate the interaction of particles with the system collimator, detector, and surroundings. In addition, the access to the canal is restricted so collecting new data to test the deconvolution technique at this time is a difficult task.

The simulated fuel source was developed by first calculating the area of the individual isotope photo-peaks $\left(\mathrm{A}_{\mathrm{pp}}\right)$ from ATR spectra. The second step in obtaining the normalized weighted fuel point source was to calculate the summation of the individual isotope photo-peaks areas $\left(\mathrm{A}_{\mathrm{pp}}\right)$ from the ATR fuel spectra in order to estimate the total weighted fuel source area $\left(\mathrm{W}_{\mathrm{ts}}\right)$

$$
W_{t s}=\sum_{n=1}^{n} A_{p p_{n}}
$$

Once the total weighted fuel source area was calculated, the individual area photo-peaks $\left(\mathrm{A}_{\mathrm{pp}}\right)$ were divided by the result of the total weighted fuel source area $\left(\mathrm{W}_{\mathrm{ts}}\right)$ to obtain the fuel individual isotope concentration (Wpp) used to simulate the surrogate source in MCNPX ${ }^{10}$

$$
W_{p p_{n}}=\frac{A_{p p_{n}}}{W_{t s}}
$$

The method describe above was used to create $\operatorname{MCNPX}^{(10)}$ spectral data for four fuel individual isotope weighted point sources representing different ATR fuel elements spectra. The MCNPX ${ }^{(10)}$ simulated fuel spectra model was based on the same geometrical setup that was describe in the experimental section.

Following the method described four simulated point weighted fuel sources were created based on $92 \% \mathrm{U}^{235}$ enriched ATR fuel spectra (Fig. 4.6 bottom) taken at the canal in 2010 as part of a study dedicated to collect burnup validation data ${ }^{6}$. The ATR fuel spectra data were collected as part of a feasibility study to gather data from the ATR 
canal adjacent to the reactor. The energy intensity and isotopic composition of the simulated point source information was extracted from ATR fuel spectra with different burnup and cooling times.

The comparison of fuel data collected at the ATR canal and the weighted simulated data can be seen in Fig. 4.6. The figure shows the comparison of element simulated and experimental data for element XA374T. The figure also demonstrates that the simulated weighted source was able to reproduce the isotopic peaks of interest in the ATR experimental spectra. The purpose of creating fuel simulated data during the study was not to have the same exact experiment setup that the ATR detection feasibility study (6) The objective of simulating highly enriched ATR fuel spectra was to create data to test if the MLEM algorithm can improve the energy resolution and decrease the standard deviation of the burnup calibration curve. There are some discrepancies between the simulated and collected spectra as expected. The differences can be attributed to the fact that the fuel simulated data did not capture the full effects of the original ATR canal experimental setup, i.e., background, minor peaks, or X-ray region. However, the simulated data were able to capture the peaks of interest needed to perform burnup calibrations and will help determine if deconvolution can aid in the prediction of fuel burnup using calibrations.

Once the simulated ATR fuel source was proven to be a good representation of the experimental fuel source, the deconvolution algorithm was applied. Fig. 4.7 shows the convoluted spectra of the surrogate ATR fuel element XA569T, while Fig. 4.8 illustrates the fuel element spectra after deconvolution. Comparing the two figures, it can be seen that the MLEM deconvolution algorithm was effective in moving the losses of energy in 
the convoluted figure to the appropriate photo-peaks in the deconvoluted plot. The deconvoluted plot also shows that the MLEM method was efficient in resolving two pair of close-lying peaks $(662,696$ and 1332,1274$)$. This also proves that deconvolution by MLEM helps resolves peaks that have a low number of counts and are lost due to pile-up or lost in the continuum. The better resolution of near lying peaks and the increase of photo-peak area in small count peaks allows for a better identification of all the isotopes present in the simulated fuel source.

In order to test the performance of the MLEM algorithm, four different surrogate element sources representing four different ATR fuel elements were modeled within $\mathrm{MCNPX}^{(10)}$. The four surrogate fuel source spectra were deconvoluted and the energy resolution for the peaks of interest $\left({ }^{134} \mathrm{Cs}\right.$ and $\left.{ }^{137} \mathrm{Cs}\right)$ was calculated. Table 4.3 shows how the energy resolution for the ${ }^{134} \mathrm{Cs}$ and ${ }^{137} \mathrm{Cs}$ improves with deconvolution for all the surrogate ATR fuel element sources. Improving the resolution of the interested peaks means that there will be less fluctuation from pulses recorded at the same energy, yielding less area uncertainty for burnup calibrations.

\section{$\underline{4.11 \text { Use of Deconvolution Spectra for Burnup Determination }}$}

The development of a deconvolution protocol for ATR fuel burnup prediction is aimed to improve the performance of the nondestructive burnup prediction technique for ATR fuels using scintillators. The deconvolution technique is designed to improve spectra quality. Improving the quality of the spectra will in theory allow for a more confident predictions as well as a broader range of operation of the 1"x1" $\mathrm{LaBr}_{3}$ scintillator for burnup calibrations and ATR canal applications. Prediction of fuel burnup 
experimentally by fuel calibrations is a technique in which certain ratios or peak areas of absolute fission products from gamma-ray spectra are used to nondestructively determine burnup. Fig. 4.9 shows an example of a burnup calibration created with experimental data collected at the ATR canal using a $\mathrm{LaBr}_{3}$ scintillator. The calibration was created using the ${ }^{137} \mathrm{Cs} /{ }^{134} \mathrm{Cs}$ area ratio versus several fuel elements calculated burnup.

The plot illustrates how the confidence in predicting burnup by this particular nondestructive method decreases for the elements with less than 200 MWd of burnup. The three elements have a cooling time of less than 100 days. Shorter cooling time of the fuel element signifies that the spectra are dominated by short-lived fission product isotopes; this makes difficult to use longer-lived ratios or absolute areas commonly used for the creation of burnup calibrations $\left({ }^{137} \mathrm{Cs}\right.$ and $\left.{ }^{134} \mathrm{Cs}\right)$. Due to the higher standard deviation of the ${ }^{137} \mathrm{Cs} /{ }^{134} \mathrm{Cs}$ area ratio of elements with short cooling times (0-100 days), there is no procedure for the future ATR permanent system to calculate burnup of these elements with confidence. The goal of the deconvoluting simulated fuel data is to prove that the standard deviation of the ${ }^{137} \mathrm{Cs} /{ }^{134} \mathrm{Cs}$ ratio can be decreased, thereby increasing the confidence in $\mathrm{LaBr}_{3}$ burnup calibration prediction, as well as increasing the cooling time range in which a calibration curve can predict burnup with a certain degree of accuracy.

With the purpose of testing the theory that deconvolution can minimize the error of burnup calibrations, two curves based on the photo-peak area ${ }^{137} \mathrm{Cs} /{ }^{134} \mathrm{Cs}$ ratios (Table 4.3) were created. The two plots represent the original four surrogate fuel sources ${ }^{137} \mathrm{Cs} /{ }^{134} \mathrm{Cs}$ ratios for the simulated data before and after deconvolution. Fig. 4.10 represents the burnup calibration curve before deconvolution, while Fig. 4.11 contains the 
area ${ }^{137} \mathrm{Cs} /{ }^{134}$ Csratios of the deconvoluted spectra. It can be deduce from the plots and from linear fit parameters as well as looking at the error bars associated with the ${ }^{137} \mathrm{Cs} /{ }^{134} \mathrm{Cs}$ ratios that the calibration plot created with the deconvolution data yields a more confident ATR fuel burnup prediction.

\section{$\underline{4.12 \text { Conclusions }}$}

The study established that the response function of a 1 "x 1 " $\mathrm{LaBr}_{3}$ detector can be calculated using MCNPX ${ }^{(10)}$. The study also established that the MLEM algorithm can be used to successfully deconvolute simulated one-isotopic data and fuel simulated generated spectra. The results show a significant improvement in the energy resolution for all the one-isotope sources along with improving the performance parameters for ${ }^{137} \mathrm{Cs}$ and ${ }^{60} \mathrm{Co}$. The results also showed that implementing the MLEM deconvolution algorithm will assist with a more precise identification of isotopes for fuel element spectra. The study also determined that the MLEM deconvolution algorithm can be effective in enhancing the energy of ${ }^{134} \mathrm{Cs}$ and ${ }^{137} \mathrm{Cs}$ peaks for four ATR fuel high enriched surrogate simulated sources. The study proves that enhancing the resolution of the ${ }^{134} \mathrm{Cs}$ and ${ }^{137} \mathrm{Cs}$ photo-peaks will help to increase the accuracy of burnup calibrations for ATR fuel using a 1"x1" $\mathrm{LaBr}_{3}$ scintillator. In conclusion, a methodology was successfully created to increase energy resolution of a scintillator that can be applied to design an efficient and more rugged fuel scanning detection system for the ATR canal for model validation and burnup on-site predictions. 


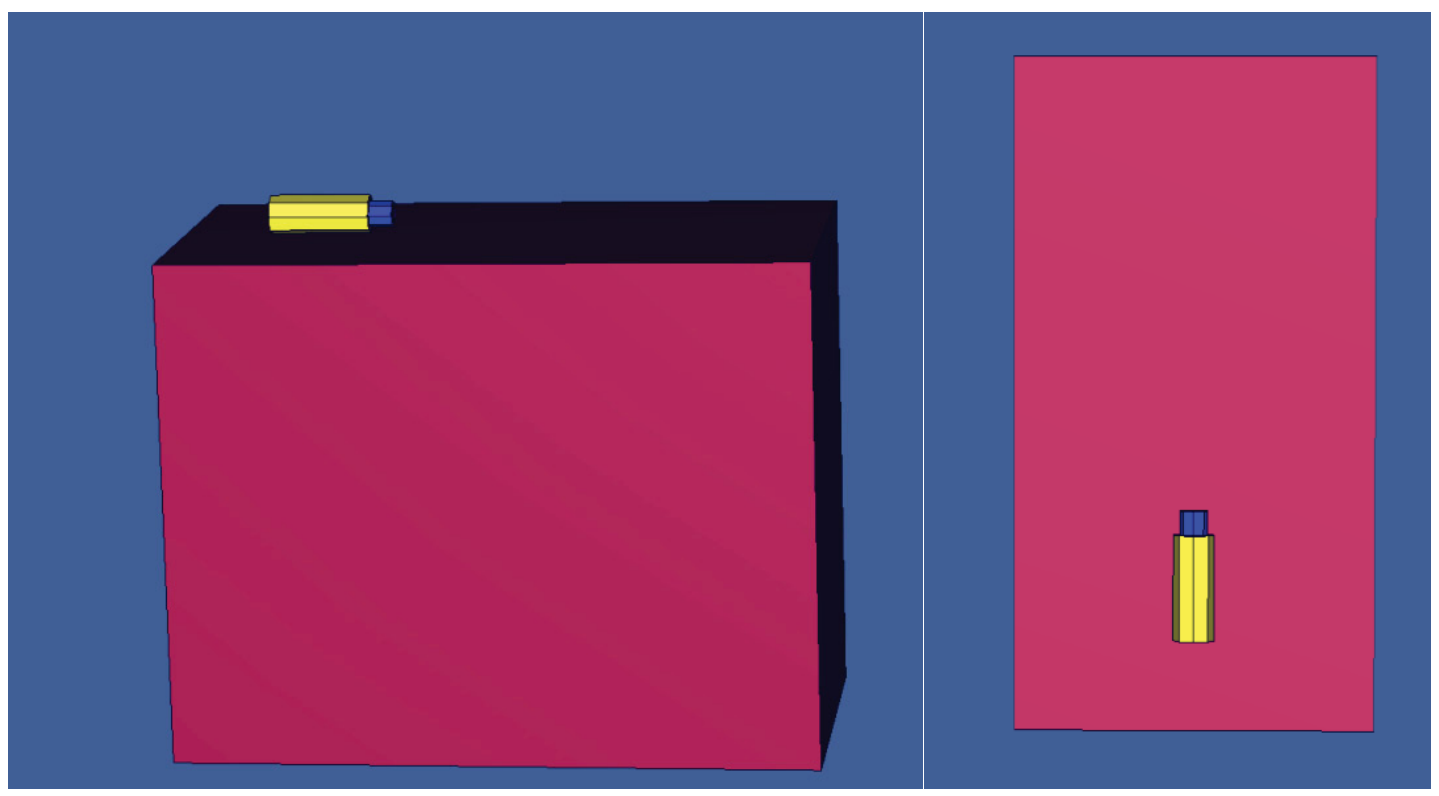

Figure 4.1 MCNPX experimental setup

Table 4.1-Gamma-energy sources used for GEB parameters

\begin{tabular}{|c|c|c|}
\hline \multirow{2}{*}{ Nuclide } & Energy $(\mathbf{K e V})$ & FWHM (keV) \\
\hline \multirow{2}{*}{ Ba K $\mathbf{\alpha}_{\mathbf{\alpha}}$ X-ray } & 32.29 & 8.25 \\
\hline Eu $^{\mathbf{1 5 2}}$ & 121.52 & 9.23 \\
\hline \multirow{5}{*}{} & 244.8 & 12.88 \\
\cline { 2 - 3 } & 344.45 & 15.39 \\
\cline { 2 - 3 } & 778.98 & 24.8 \\
\cline { 2 - 3 } & 867.08 & 27.47 \\
\cline { 2 - 3 } & 964.01 & 26.96 \\
\hline \multirow{2}{*}{$\mathbf{C s}^{\mathbf{1 3 7}}$ (662 peak) } & 1408.01 & 39.17 \\
\hline
\end{tabular}




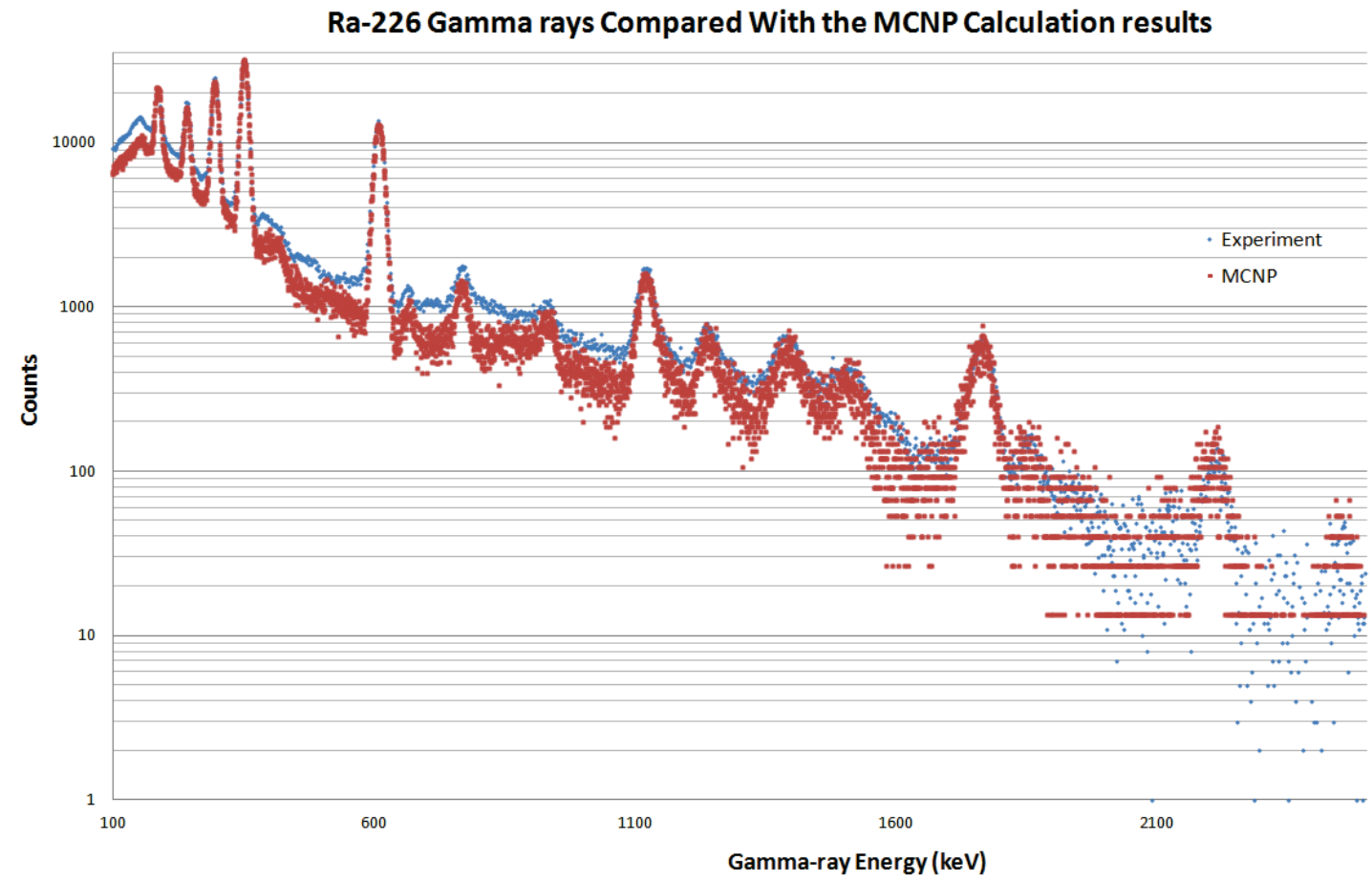

Figure 4.2 ${ }^{226} \mathrm{Ra}$ experimental and simulated MCNP spectra 


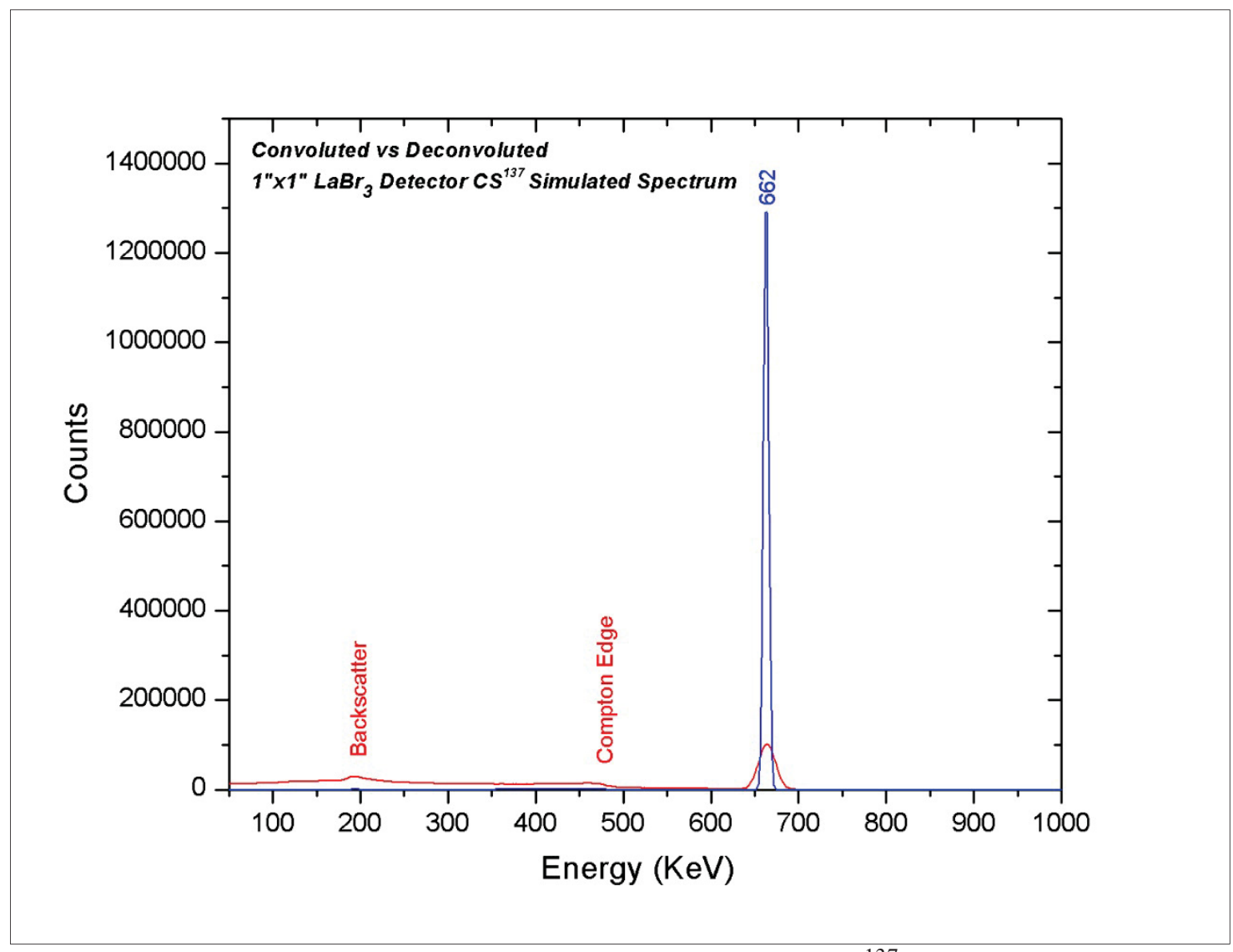

Figure 4.3 Simulated convoluted spectrum of a ${ }^{137} \mathrm{Cs}$ source 


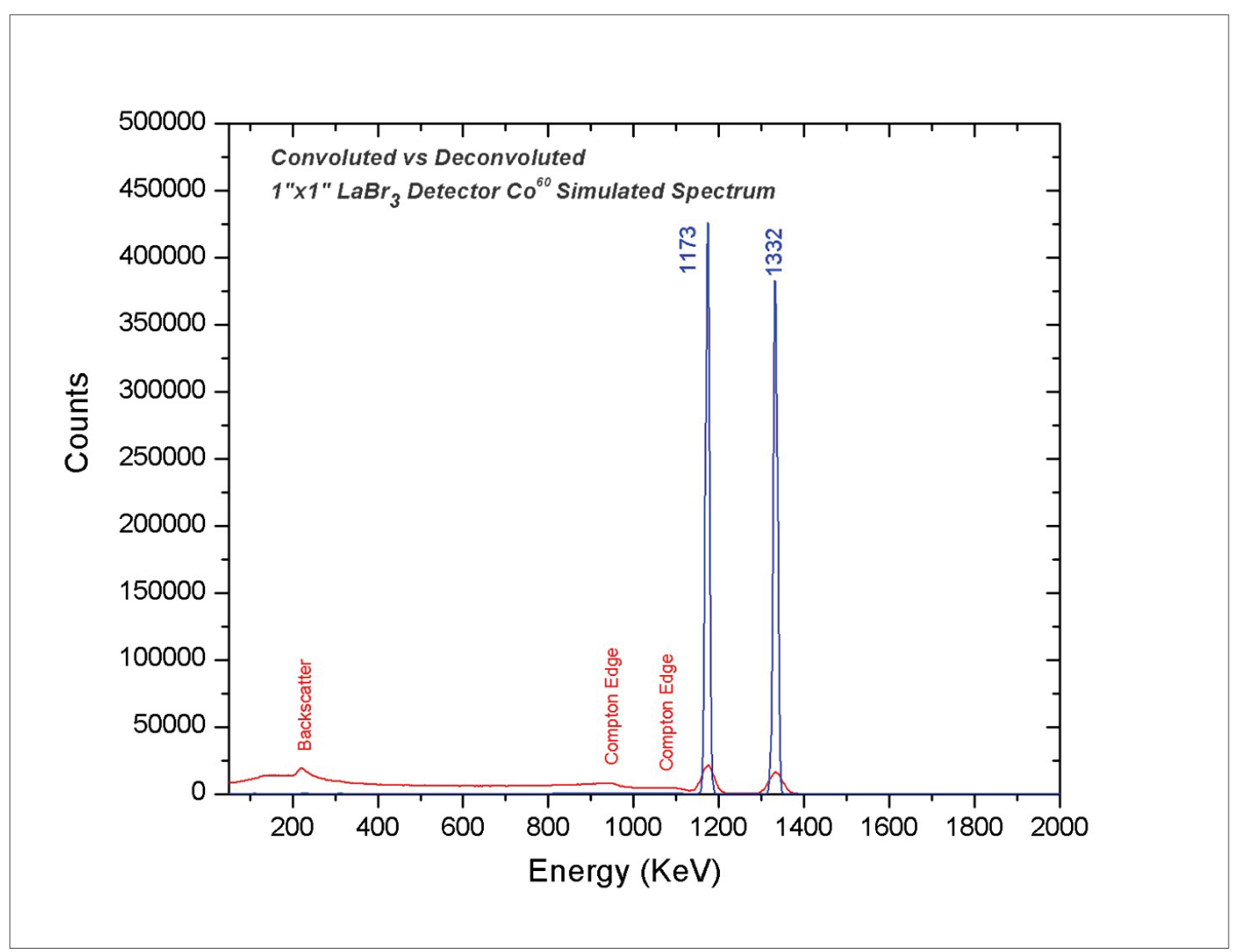

Figure 4.4 Simulated convoluted spectrum of a ${ }^{60} \mathrm{Co}$ source 


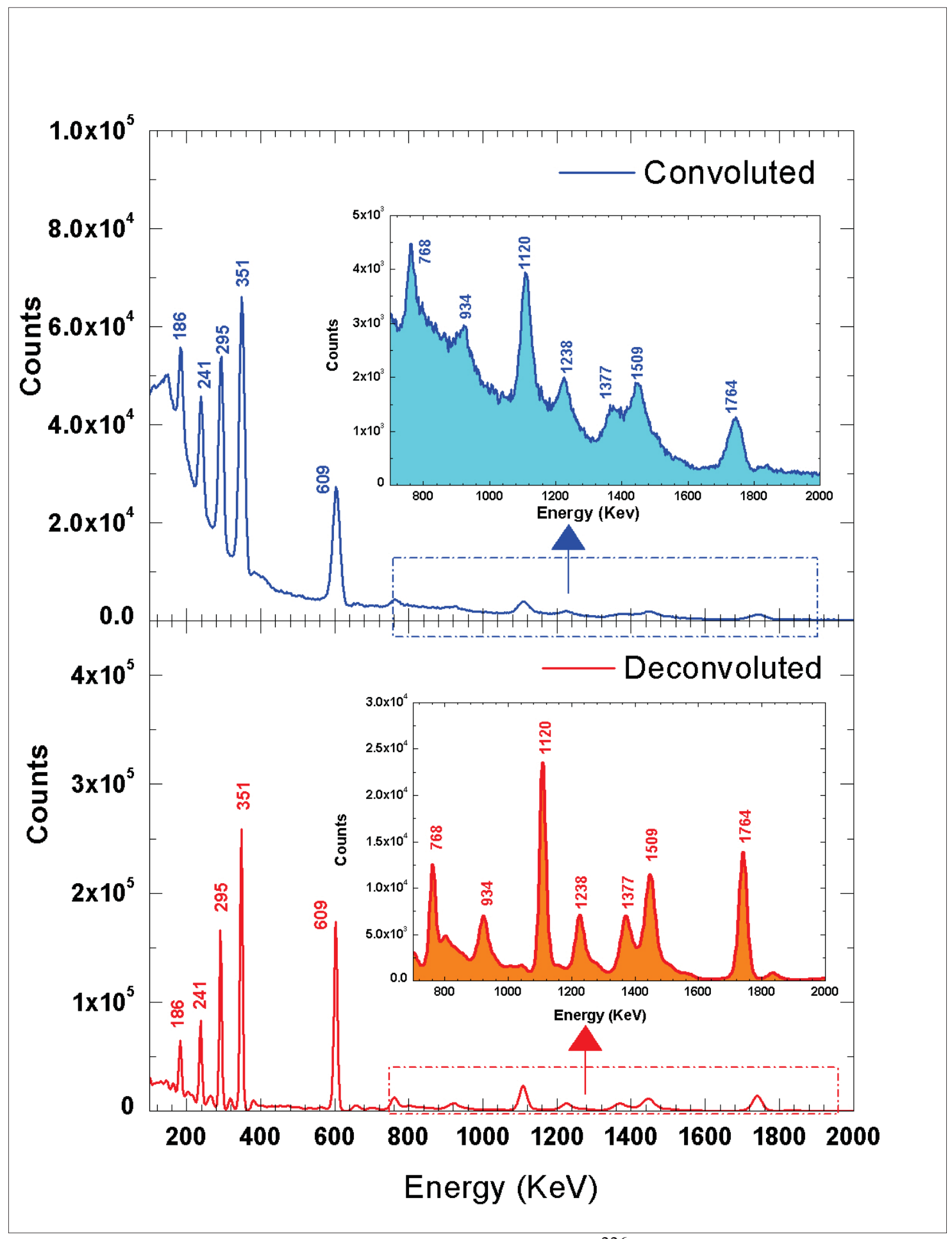

Figure 4.5 Simulated spectrum of $\mathrm{a}^{226} \mathrm{Ra}$ source 
Table 4.2-Simulated one-isotope deconvolution performance measurements

\begin{tabular}{|c|c|c|c|c|c|}
\hline Source & $\begin{array}{c}\text { Peak } \\
\text { Energy } \\
(\mathrm{KeV})\end{array}$ & Spectrum & $\begin{array}{c}\text { Peak } \\
\text { Resolution } \\
(\%)\end{array}$ & Peak/Compton & $\begin{array}{l}\text { Peak } \\
\text { /Total }\end{array}$ \\
\hline \multirow[b]{2}{*}{${ }^{137} \mathrm{Cs}$} & \multirow[b]{2}{*}{662} & $\begin{array}{l}\text { Simulated } \\
\text { Spectrum }\end{array}$ & 3.35 & 8.43 & 0.49 \\
\hline & & $\begin{array}{c}\text { Simulated } \\
\text { Deconvolution }\end{array}$ & 1.05 & 6297.22 & 2.05 \\
\hline \multirow[b]{4}{*}{${ }^{60} \mathrm{Co}$} & \multirow[b]{2}{*}{1173} & $\begin{array}{l}\text { Simulated } \\
\text { Spectrum }\end{array}$ & 2.92 & 3.66 & 0.15 \\
\hline & & $\begin{array}{c}\text { Simulated } \\
\text { Deconvolution }\end{array}$ & 0.92 & 24402.04 & 0.99 \\
\hline & \multirow[b]{2}{*}{1332} & $\begin{array}{l}\text { Simulated } \\
\text { Spectrum }\end{array}$ & 2.81 & 3.78 & 0.13 \\
\hline & & $\begin{array}{c}\text { Simulated } \\
\text { Deconvolution }\end{array}$ & 0.87 & 1186.33 & 0.95 \\
\hline \multirow[b]{4}{*}{${ }^{226} \mathrm{Ra}$} & \multirow[b]{2}{*}{351} & $\begin{array}{l}\text { Simulated } \\
\text { Spectrum }\end{array}$ & 4.41 & - & - \\
\hline & & $\begin{array}{c}\text { Simulated } \\
\text { Deconvolution }\end{array}$ & 0.98 & - & - \\
\hline & \multirow[b]{2}{*}{609} & $\begin{array}{l}\text { Simulated } \\
\text { Spectrum }\end{array}$ & 3.45 & - & - \\
\hline & & $\begin{array}{c}\text { Simulated } \\
\text { Deconvolution }\end{array}$ & 1.09 & - & - \\
\hline
\end{tabular}




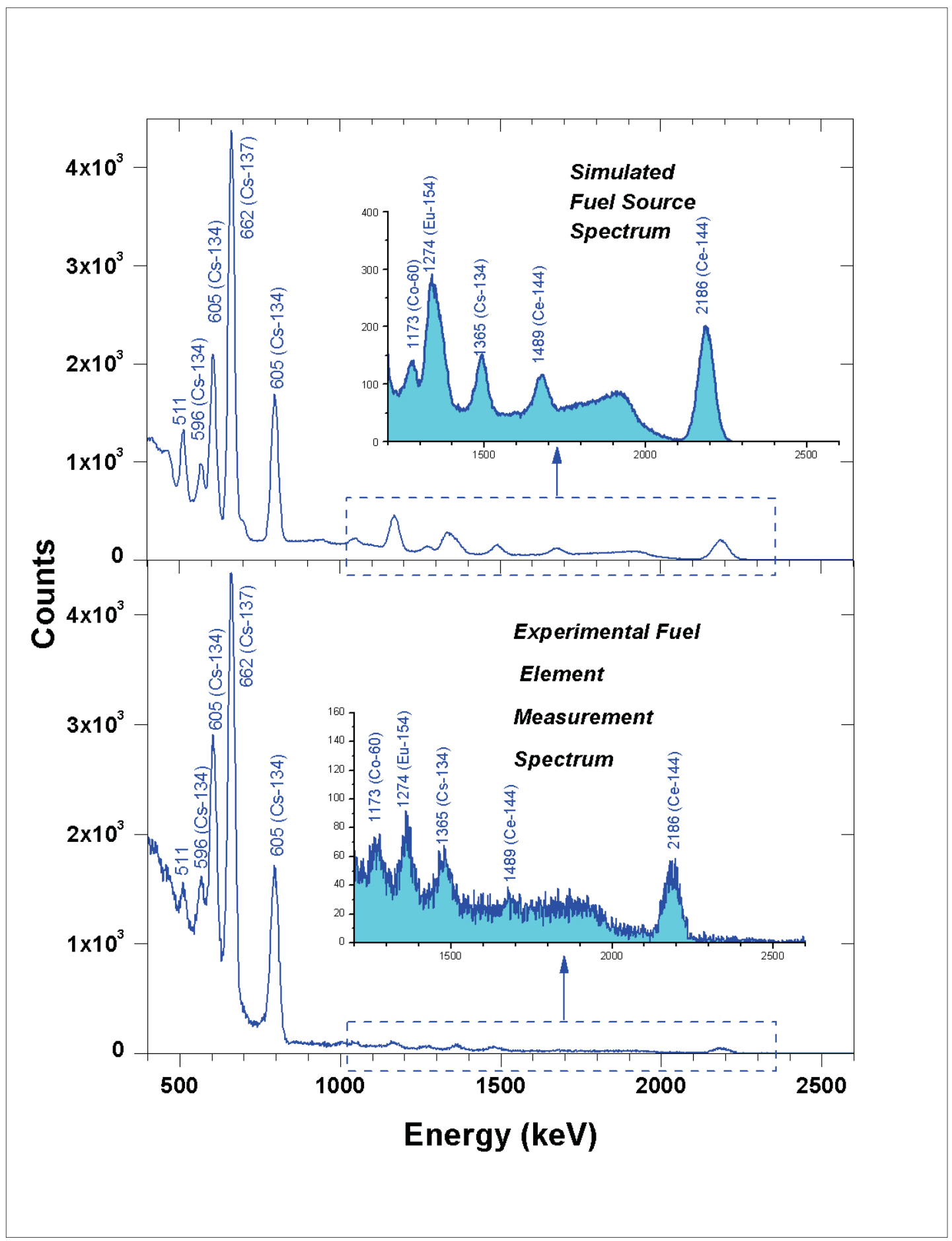

Figure 4.6 Experimental and simulated fuel ATR spectra comparison 


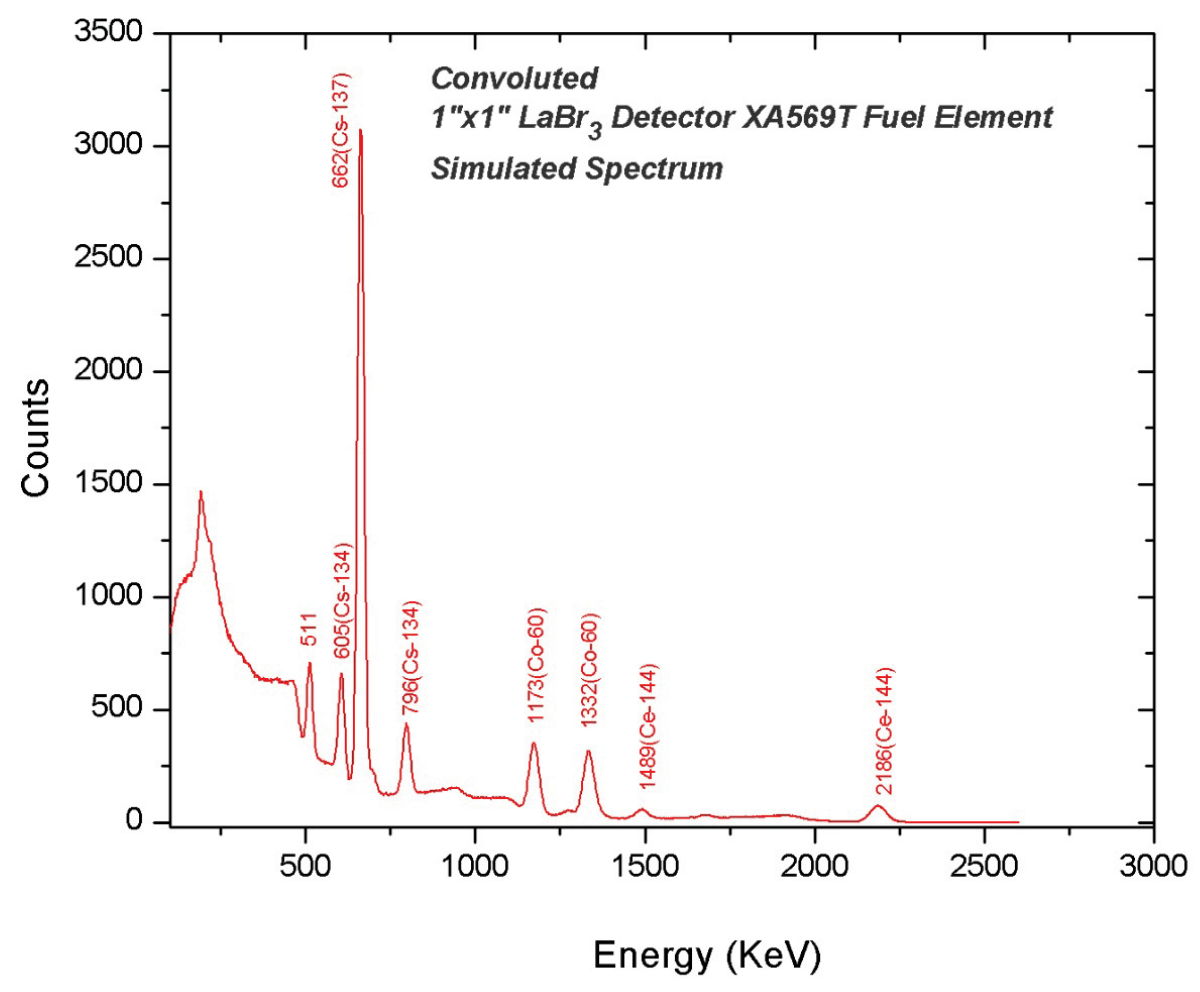

Figure 4.7 Convoluted simulated fuel ATR spectra 


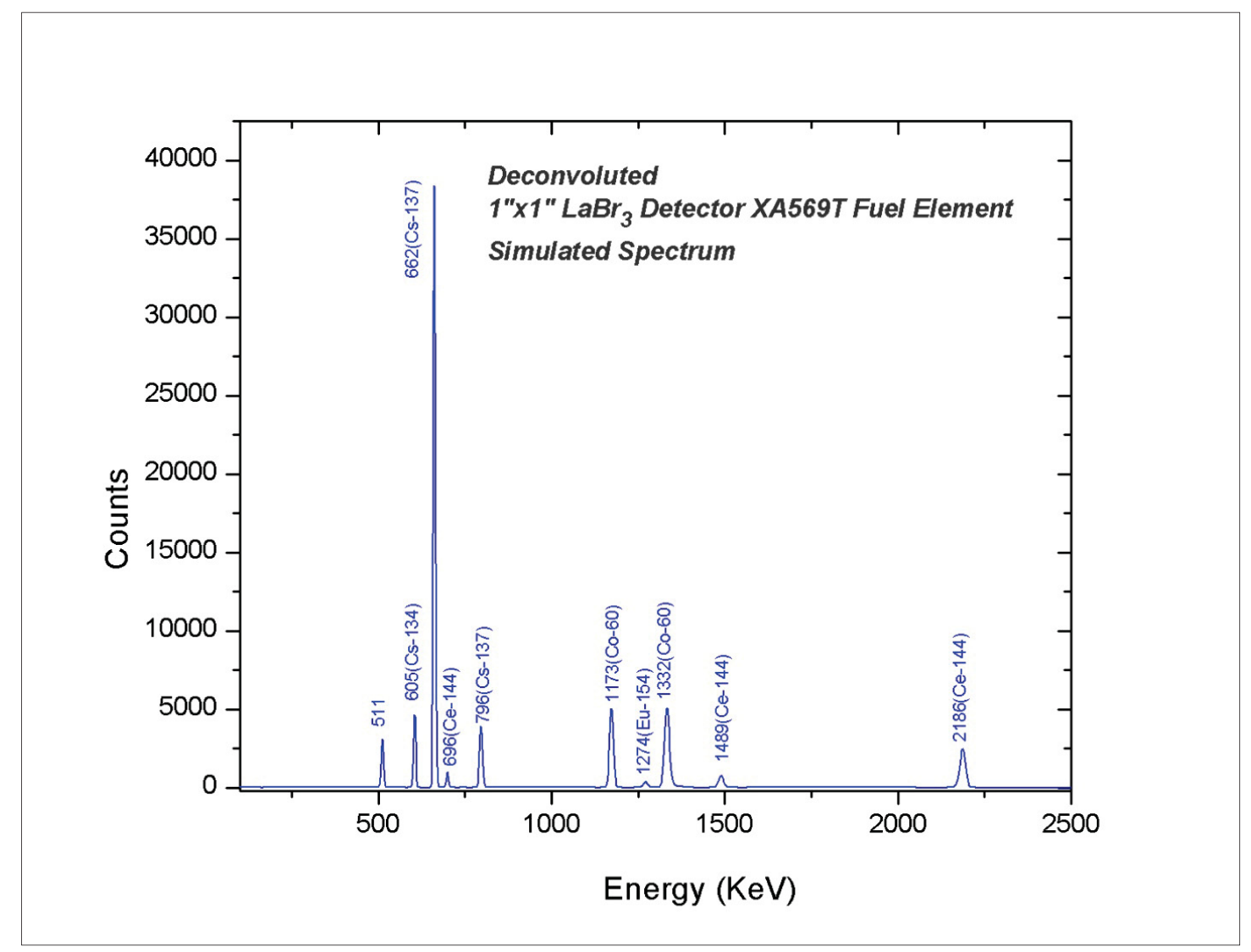

Figure 4.8 Deconvoluted simulated fuel ATR spectra

Table 4.3. Surrogate fuel elements photo-peaks resolution

\begin{tabular}{|c|c|c|c|c|}
\hline & \multicolumn{2}{|c|}{ Convoluted } & \multicolumn{2}{c|}{ Deconvoluted } \\
\hline Element & $\begin{array}{c}\text { Peak } \\
\text { Resolution } \\
\text { Cs }^{\mathbf{1 3 4}}\end{array}$ & $\begin{array}{c}\text { Peak } \\
\text { Resolution } \\
\text { Cs }^{\mathbf{1 3 7}}\end{array}$ & $\begin{array}{c}\text { Peak } \\
\text { Resolution } \\
\text { Cs }^{\mathbf{1 3 4}}\end{array}$ & $\begin{array}{c}\text { Peak } \\
\text { Resolution } \\
\text { Cs }^{\mathbf{1 3 7}}\end{array}$ \\
\hline Xa569T & 3.59 & 3.36 & 1.23 & 1.25 \\
\hline Xa377T & 3.58 & 3.29 & 1.48 & 1.18 \\
\hline Xa374T & 3.55 & 3.34 & 1.24 & 1.1 \\
\hline Xa379T & 3.36 & 3.28 & 1.54 & 1.31 \\
\hline
\end{tabular}




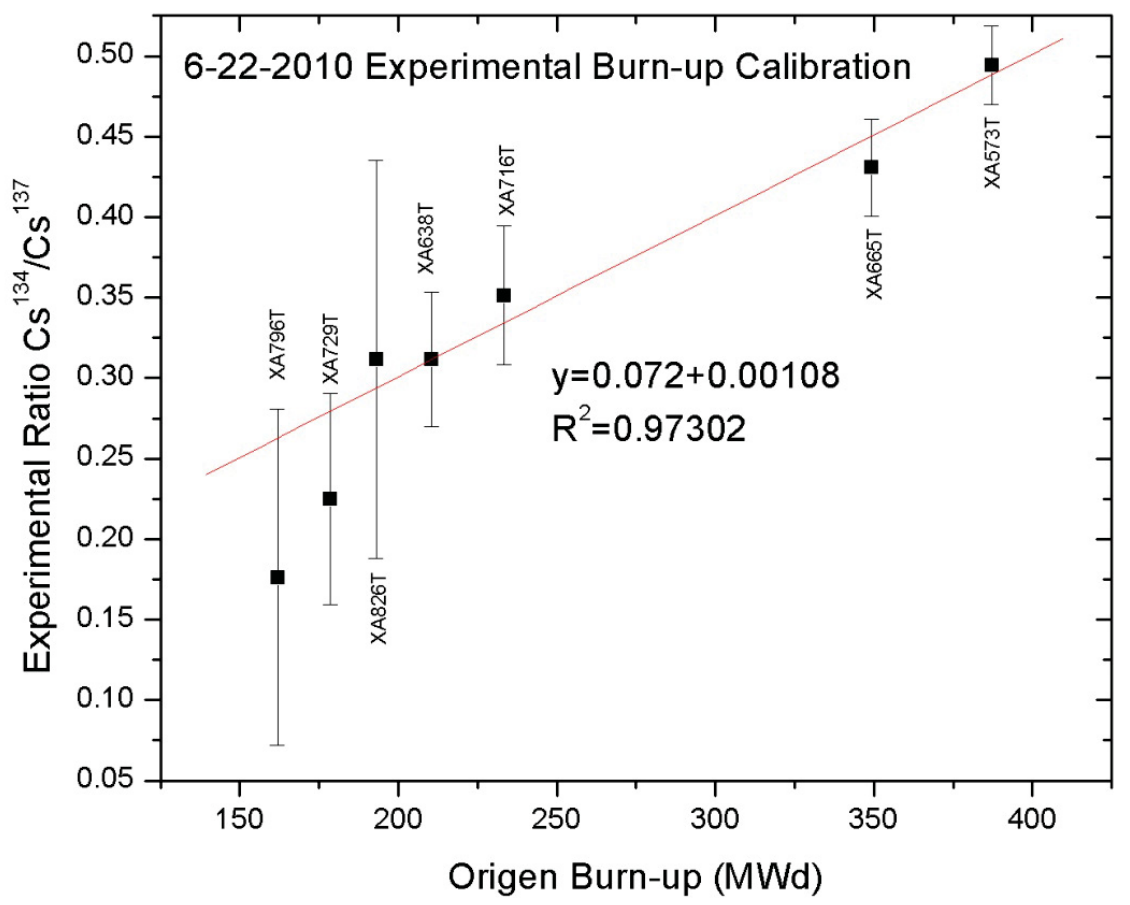

Figure 4.9 Example of an ATR fuel burnup prediction calibration 


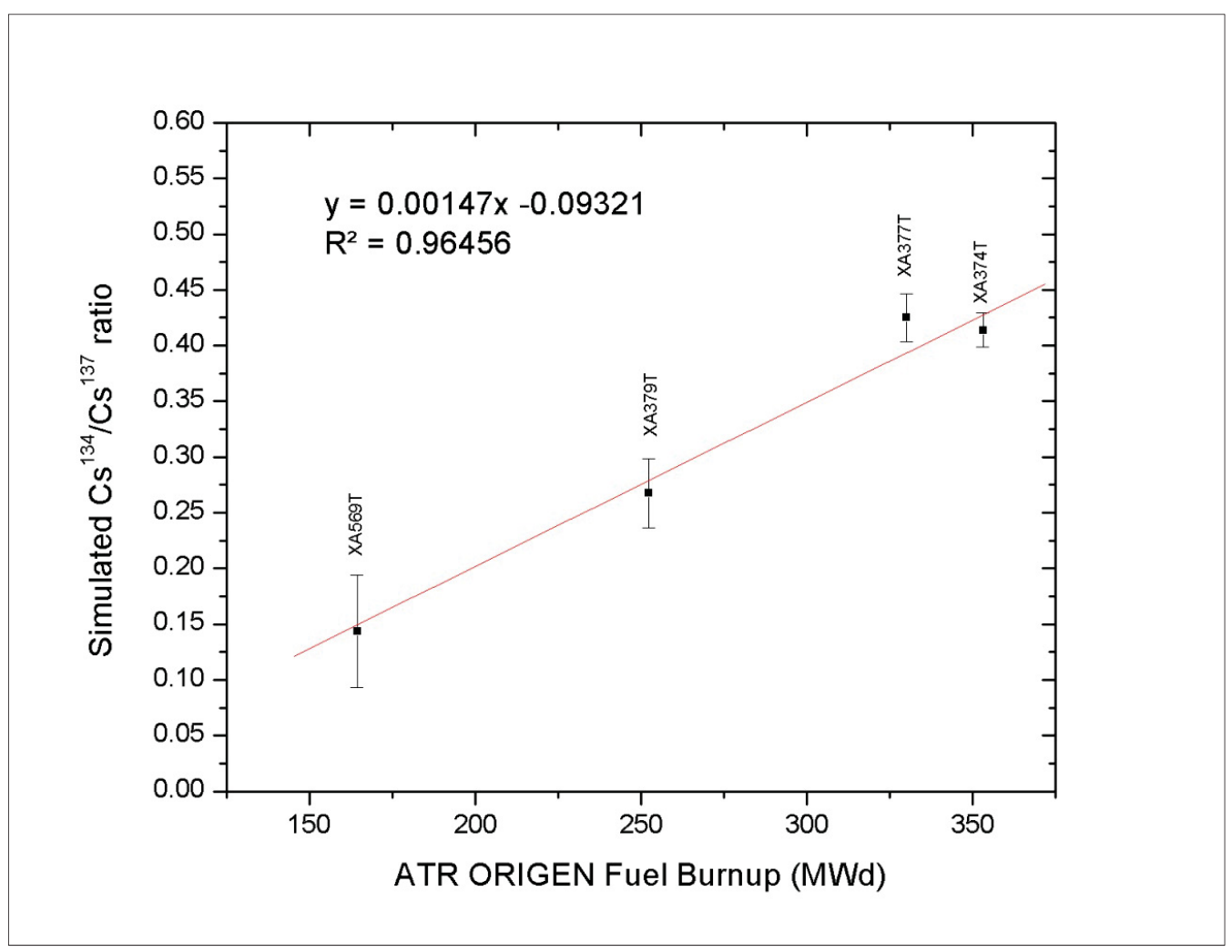

Figure 4.10- Convoluted burnup calibration simulated fuel ATR spectra 


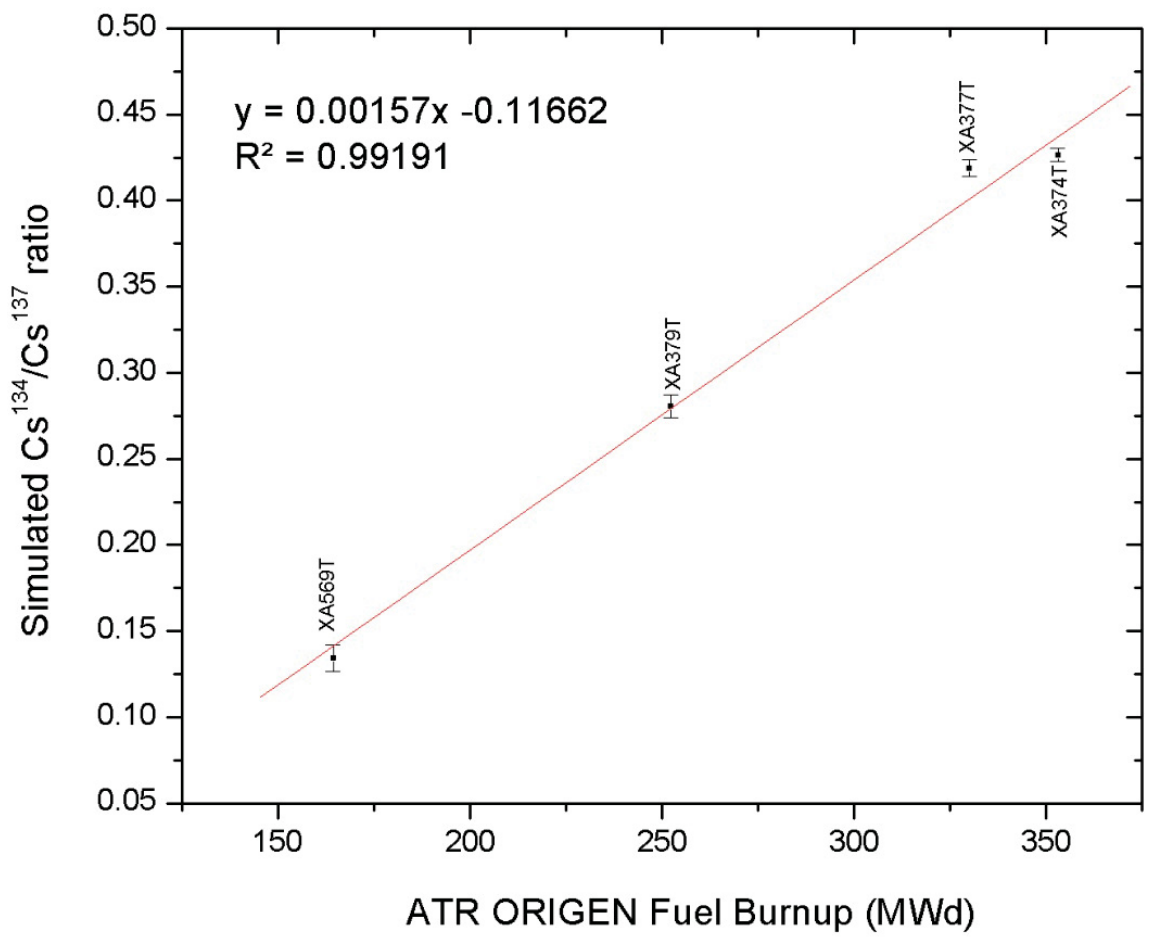

Figure 4.11 Deconvoluted burnup calibration simulated fuel ATR spectra 


\section{$\underline{4.13 \text { References }}$}

1 Meng, L.J.; Ramsden, D. An Inter-comparison of Three Spectral-Deconvolution Algorithms for Gamma-ray Spectroscopy, IEEE Transactions on Nuclear Science, 2000,Vol. 47, No. 4, 1329-1336.

2 Mertens, C.; et al. MCNP simulation and Spectrum Unfolding for an NAI Monitor of Radioactivity in Aquatic Systems,

3 Hakimabad, H. M., et al. Evaluation the Nonlinear Response Function of a3 x 3 in $\mathrm{NaI}$ Scintillation Detector for PGNAA Applications. Applied Radiation and Isotopes, 2007, Vol 65, 918-926.

4 Zeballos-Chavez, J.Y.; et al. Response Function of a Germanium Detector to Photon Energies Between 6 and $120 \mathrm{KeV}$. Nuclear Instruments and Methods in Physics Research, 2001, Vol 457, 212-219.

5 Kovaltchouck, V.; Machrafi, R. MonteCarlo Simulations of response for Gas Filled and Scintillator detectors with MCNPX Code. Annals of Nuclear Energy, 2011, Vol. $38,788-793$.

6 Navarro, J.; Aryaeinejad, R.; Nigg, D.W. A Feasibility Study to Determine Cooling Time and Burnup of Advanced Test Reactor Fuel Using a Nondestructive Technique and Three Types of Gamma-ray Detectors. Journal of ASTM International, 2012, Vol. 9, Issue 3.

7 Maximum-Likelihood Expectation-Maximization (ML-EM) Iterative Reconstruction Algorithm, UC Berkley.

8 Monahan, J.E. Unfolding Measured Distributions. Scintillation Spectroscopy of Gamma Radiation, Shafroth, S. M.; Gordon and Breach Science Publishers Inc.New York, 1967, 371-386.

9 X-5 Monte Carlo Team, April 2003A general Monte Carlo N-Particle Transport Code, Version 5, Overview and Theory, Vol. 1, pp. 106-107, Los Alamos, NM.

10 Pelowitz, D. B. MCNPX A General N-Particle Transport Code, version 2.6.0”. LACP-07-1473, Los Alamos, NM.

11 Shepp, L.A.; Vardi, Y.; Maximum Likelihood Reconstruction for Emission Tomography. IEEE Trans. Medical Imaging, 1982, Vol 1(2), 113-122.

12 Bryan, R. K; Skilling, J. Deconvolution by Maximum Entropy, as Illustrated by Application to the Jet of M87 1987, Mon. Not. R. Astr. Soc.,191,69-79. 
13 McElroy, W. N.; Berg, S. Crockett T.; Hawkings, R. G. A computer-automated iterative method for neutron flux spectra determination by foil activation, U.S. Air Force Weapons Laboratory;AFWL-TR-67-41; Kirkland AFB, NM, 1967. 


\section{CHAPTER 5}

\section{GAMMA-RAY SPECTRA DECONVOLUTION OF EXPERIMENTAL DATA}

The previous chapter established that applying deconvolution to $\mathrm{LaBr}_{3}$ scintillator simulated data improves not only the resolution of the photo-peaks and performance spectra parameters but also improves the confidence of the burnup calibration curves; however, the method was only tested using simulated data. In order to better determine the performance of the method, the method has to be tested using experimental data. The final objective of establishing a deconvolution process for the $\mathrm{LaBr}_{3}$ spectra is to be able to efficiently use this detector in the final design of the ATR fuel scanning system. However, it was not feasible to test this method with the experimental fuel spectra already collected because with the system configuration used for the ATR canal measurements, it was not possible to determine the system geometry with a high degree of accuracy. Knowing the geometry of the system with precision is necessary to accurately compute the response function of the detector. In order to demonstrate that this technique will ultimately help to increase the resolution of spectra collected with a $\mathrm{LaBr}_{3}$ detector for fuel measurements applications, a surrogate fission product source was created. 
Chapter 6 presents the final step of this study that consisted in developing a highly enriched source using fission wires as a surrogate fuel source. The surrogate fuel was created using ATR fission wires that have the same uranium enrichment as the ATR fuel elements. The surrogate source was irradiated at the AGN-201 reactor at Idaho State University. The surrogate fuel source was placed in the reactor for about 20 minutes and then the samples were taken to a laboratory where data were collected using the $\mathrm{LaBr}_{3}$ detector at various decay intervals. After the data were collected, the deconvolution method developed in Chapter 4 was applied initially to one- and multiisotopic sources, and then the technique was tested using the multiple decay spectra of the surrogate fission source. 


\title{
CHAPTER 6
}

\author{
MAXIMUM LIKELIHOOD EXPECTATION MAXIMIZATION \\ ALGORITHM SPECTRAL DECONVOLUTION OF A 1" X 1” \\ $\mathrm{LaBr}_{3}$ GAMMA-RAY DETECTOR FOR ONE-ISOTOPE, \\ MULTI-ISOTOPE, AND FISSION PRODUCT \\ SOURCES
}

\begin{abstract}
$\underline{6.1 \text { Abstract }}$
An experiment to irradiate highly enriched samples in order to create a multipeak isotope source to test a deconvolution method for a $\mathrm{LaBr}_{3} 1$ "x 1 " detector was performed. The samples irradiated were ten fission standard wires normally used to measure fission rates at the Advanced Test Reactor located at the Idaho National Laboratory. The ten highly enriched fission wires $\left(93 \%{ }^{235} \mathrm{U}\right)$ were irradiated at a maximum power of 4.6 watts for two hours corresponding to a $2.3 \times 10^{8} \mathrm{n} / \mathrm{cm}^{2}$ s neutron flux environment. After the irradiation of the sample, several measurements were performed at a laboratory adjacent to the reactor. Following the spectral data collection, the deconvolution method was applied to one- and multi-isotope sources as well as to the highly enriched fission product sample.
\end{abstract}




\section{$\underline{6.2 \text { Introduction }}$}

There is an ongoing effort to design a permanent fuel scanning system for the ATR canal. The permanent gamma scanning system will be used to predict burnup nondestructively and to collect fuel validation data needed for the new suite of codes that will be implemented for ATR operations. The first stage of the design process of a fuel scanning system was to perform a feasibility study at the ATR canal in order to determine if meaningful gamma spectroscopy data can be extracted from the high radiation environment along with determining if predicting burnup on-site for ATR fuels was possible. The detectors used during the study were HPGE, $\mathrm{LaBr}_{3}$, and HPXE. The feasibility study determined that meaningful data in an above the water configuration could be obtain with the three detectors. The study also determine that burnup calibration curves could be perform with data extracted from the spectra taken with the three different detectors; however, the $\mathrm{LaBr}_{3}$ and the HPGE burnup curves outperform the HPXE calibrations ${ }^{2}$.

After the feasibility study was successfully completed, a preliminary design for the permanent system had to be performed. The preliminary design included determining the primary detector for the ATR fuel permanent scanning system. In order to determine which detector was better suited for the ATR fuel scanning system, several factors had to be taken into account. Among those factors is the location of the permanent system. The location of the permanent system is a design limitation. The ATR canal is a high radiation facility where material are brought in and out of the canal at a constant rate. The work pace of the ATR canal is fast and situations are ever evolving. Therefore, the system has to be rugged in order to withstand the daily activities of the working spent fuel canal and 
it also needs to be as low maintenance as possible as supporting the system will not always be a priority. If the fuel scanning system location was in a laboratory or in a more controlled setting the HPGE detector would be without any disagreement the ideal primary detector. However, since this detection system will be located at the ATR canal, the detector of choice has to be able to endure the harsh ATR operation conditions. Taking these factors into account, the HPGE detector was not the ideal choice for this system as a primary detector. The HPGE detector will still be available for specific tasks, but for routine fuel scanning, a detector that requires less maintenance and that can better withstand the daily activities of the ATR canal is needed.

From the other two detectors tested during the study, the $\mathrm{LaBr}_{3}$ quality of spectra collected was superior to the spectra gathered using the HPXe detector. Furthermore, the $\mathrm{LaBr}_{3}$ calibration curves prediction confidence was very similar to the HPGE calibration curves performance. For these reasons along with the fact that the $\mathrm{LaBr}_{3}$ detector is well suited for in-situ measurement and that it also requires very little maintenance, the $\mathrm{LaBr}_{3}$ was considered the primary option for the ATR permanent fuel scanning system. However, with all the upsides that the $\mathrm{LaBr}_{3}$ scintillator has for on-site measurements, the peak energy resolution of the detector is significantly below the HPGE resolution. Consequently and before implementing the $\mathrm{LaBr}_{3}$ as a primary detector of the permanent system, a study was performed in order to develop a protocol to increase the resolution of the $\mathrm{LaBr}_{3}$ scintillator ${ }^{7}$.

The results of the deconvolution study were successful ${ }^{11}$; however, the energy resolution enhancement protocol was only tested using simulated sources. In order to test the deconvolution protocol for the 1 "x1" $\mathrm{LaBr}_{3}$ detector using multiproduct isotopic 
sources, an experiment to irradiate fission wires that contain $10 \%$ of uranium of which $93 \%$ is ${ }^{235} \mathrm{U}$ was developed. The experiment was performed at the AGR reactor at Idaho State University. After the fission source was created, several measurements were performed. Once the spectra were collected, the protocol previously developed for the 1 1"x1" $\mathrm{Labr}_{3}$ detector was tested.

The deconvolution protocol consisted of setting up an experiment were the geometry is accurately known, collecting data, simulating and calibrating an MCNPX model of the experiment setup, calculating the response function of the detector using $\mathrm{MXNPX}^{(5)}$, and finally applying the MLEM deconvolution algorithm to the spectra source data collected using the 1 " $x 1$ " $\mathrm{LaBr}_{3}$ scintillator.

\subsection{Experimental Measurement Setup}

Fig. 6.1 and 6.2 show the experimental measurement setup used to collect data. The setup consisted of a 1 inch diameter aperture bismuth collimator and the 1 " $x 1$ " $\mathrm{LaBr}_{3}$ detector surrounded by a lead shield. The bismuth collimator has a length of $10.1 \mathrm{~cm}$ and was placed next to the detector, as can be seen in Fig. 6.1. To perform the calibration experiments one-isotope and multi-isotopes sources along with the irradiated sample were placed at the end of the bismuth collimator. The lead shielding's (Fig. 6.2) purpose was to decrease the background along with minimizing the exposure to the irradiated sample. 


\section{$\underline{6.4 \text { Detector and Simulation Calibration Measurements }}$}

The first stage of the study was to create an experimental measurement setup in a laboratory adjacent to the reactor. However, before performing the measurements with the multifission product isotopic source, calibration measurements were performed. The calibration measurements consisted of collecting data from one- and multiple-isotopes sources (Table 6.1) using the experimental setup discussed in the previous section. The data collected were used to validate the MXNPX model, perform the energy calibration of the detector, along with obtaining the parameters needed to give the MCNPX simulations pulse height data with a realistic Gaussian photo peak shape.

MCNPX contains a tally option named Gaussian Energy Broadening (GEB) that allows the user to input experimental data in the form of eq. $6.1^{(4)}$.

$$
F W H M=a+b \sqrt{E+c E^{2}}
$$

The parameters from eq. 6.1 are obtained using the full width at half maximum (FWHM) calibration data from the different sources in Table 6.1 and performing a nonlinear regression. The GEB prameters calculated for this study are:

$$
a=-0.0001827 \mathrm{MeV} \quad b=0.0271209 \mathrm{MeV}^{1 / 2} \quad c=0.7812452 \mathrm{MeV}^{-1}
$$

Once the GEB parameters for the detection system were calculated, a model of the experimental setup was created in order to simulate the different calibration sources shown in Table 6.1. After the simulations were completed, the model spectra generated were compared against the experimental data collected from three one-isotope sources. The results of the comparisons between experimental data and simulated data obtained using MCNPX are shown in Fig. 6.3-6.5. 
The three comparison figures presented for ${ }^{137} \mathrm{Cs},{ }^{60} \mathrm{Co}$, and ${ }^{22} \mathrm{Na}$ (Fig. 6.3-6.5) show that the simulated spectra created using MCNPX are in overall good agreement with the experimental data and therefore are an accurate representation of the experimental setup. The simulated spectra only diverge from the experimental data at lower energies around the Compton region. The differences are more noticeable for the

${ }^{137} \mathrm{Cs}$ and ${ }^{22} \mathrm{Na}$ sources. These deviations can be mainly attributed to the contributions of the X-ray emissions from the collimator and shielding materials surrounding the detector. The deviations can also be caused by modeling geometry and method discrepancies.

\section{$\underline{6.5 \text { Response Function Calculation }}$}

Once a realistic model of the experimental setup was validated, the response function of the $\mathrm{LaBr}_{3}$ detector was calculated. The response function of the detector can be calculated experimentally, using correlations or using simulation tools ${ }^{1}$. Simulations are the most accurate technique of calculating the response function of a detector, if a good model representation of the experimental setup is available ${ }^{1}$. The response function of the 1 "x1" $\mathrm{LaBr}_{3}$ scintillator was calculated by modeling 1024 mono-energetic sources in MCNPX. Each simulation was performed with $1 \times 10^{10}$ particles using 32 processors. This method of calculating the response function can be very accurate but is computerintensive and time-consuming. The simulations were performed using the High Performance Computer cluster at the Idaho National Laboratory. 


\subsection{Maximum Likelihood Expectation Maximization (MLEM) Algorithm}

The collection of spectral data by a gamma spectrometer can be described mathematically as:

$$
M(E)=\int_{0}^{\infty} R\left(E, E_{o}\right) S(E)
$$

where $M(E)$ is the measured spectrum, $R\left(E, E_{o}\right)$ is the response functions of the gamma spectrometer at various energy intervals, and $S(E)$ is the true gamma-ray spectrum emitted by a radioactive source. The true signal emitted by a source cannot be capture completely due to the inefficiencies and lack of resolution of the system.

The integral representing the collection of spectral data can be discretize in order to find a numerically solution

$$
\begin{aligned}
& M=R S
\end{aligned}
$$

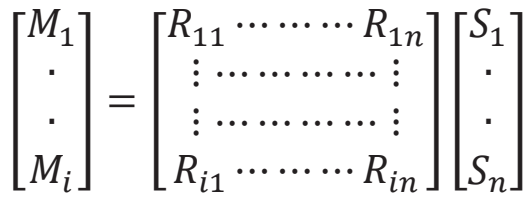

where $M_{i}$ represents the true detector counts, $R_{i}$ is the response function of the detector, and $S_{i}$ represents the actual spectrum counts. The simplest way to solve eq. 6.4 would be by simply inverting the response function of the detector.

$$
S=R^{-1} M
$$

However, the response function matrix for gamma detection applications is a sparse matrix. Because of the sparseness of the matrix, several methods are available to find the true counts of the gamma detection system. For this study, the Maximum Likelihood Fitting by Expectation Maximization (MLEM) was chosen, for the reason that it had previously shown success deconvoluting low-resolution scintillator spectra even when the peaks are small and have few counts ${ }^{1}$. 
The MLEM algorithm is an iterative method originally developed for reconstruction of topographic images ${ }^{6}$. The method assumes a Poisson distribution for each independent variable, where $M_{j}$ is the measured spectrum by the gamma detector and $R_{j i}$ is the response function of detection syestem. In this algorithm, it is assumed that $x_{i}^{(n)}$ is the best estimate of the actual gamma-ray spectrum ${ }^{4}$.

$$
x_{i}^{(n+1)}=x_{i}^{(n)}\left\{\frac{1}{\sum_{i=1}^{j} R_{j i}} \sum_{i=1}^{j}\left[\frac{M_{j}}{\sum_{k=1}^{K} R_{j k} x_{k}^{(n)}} R_{j i}\right]\right\}
$$

The algorithm calculates a new value for $x_{i}^{(n+1)}$ in each iteration and it continues until it reaches a user-specified tolerance value. The tolerance value for this study was calculated using the mean squared difference between consecutive iterations.

\subsection{MLEM Deconvolution of One-Isotope Sources}

The MLEM method described in the previous section was implemented in Matlab. To test the deconvolution algorithm, various one-isotopic sources measured using the experimental setup described in section 6.3 were deconvoluted, as can be seen in Fig. 6.6, 6.7, and 6.8.

The three one-isotope source comparison figures (6.7-6.9) showed the improvement in energy resolution for all the peaks in each one of the spectra. There are small discrepancies for the three figures that are located around the Compton region. The discrepancies can be attributed to the response function simulations difficulty of capturing the model X-ray region with complete accuracy, as was shown in the validation of the experimental setup figures (Fig. 6.3-6.5). 
In order to measure the efficiency of deconvolution method, the peak-to-Compton and peak-to-total ratios were measured. In addition, the resolutions of all the photo-peaks for the three one-isotope sources were also calculated. The comparison of the performance parameters used to measure the efficiency of the deconvolution method can be seen in Table 6.2. The table shows improvement in both performance parameters along with the improvement of resolution for all the sources. The table shows that for all three sources, the resolution improves more than $50 \%$. The table also shows that the peak-to-Compton ratio improves for the three sources; the increase of this performance parameter means that the energy losses to the Compton scattering due to inefficiencies in the detector are properly replaced in the photo-peak during deconvolution.

\section{$\underline{\text { 6.8 MLEM Deconvolution of Multiple-Isotope Source }}$}

Once it was determined that the MLEM method was an effective method deconvoluting one-isotope sources, the method was tested with a multi-isotope source. The multi-isotope source consisted of a ${ }^{152} \mathrm{Eu},{ }^{154} \mathrm{Eu}$, and ${ }^{155} \mathrm{Eu}$ mixture. Before deconvoluting the multi-isotope europium source, experimental data were smoothed to minimize the contribution that noisy data has on creating small artificial peaks. The filter used was a nine point frame quartic Savitzky-Golay filter. After the filter was applied, the data were deconvoluted using the MLEM algorithm implemented in Matlab.

Fig. 6.9 shows the deconvolution comparison of the europium multi-isotope source before and after deconvolution. In Fig. 6.9, it can be seen that the deconvolution algorithm has an overall good performance. In addition, the deconvoluted plot shows that the MLEM method was able to resolve close-lying peaks. In the plot, it can be seen that 
the near isotopic peaks 105 and $122 \mathrm{KeV}$ were fully resolved in the deconvoluted figure. In addition, the close-lying peaks 723 and $778 \mathrm{Kev}$ in the deconvoluted plot were also better resolved than in the convoluted figure. The convoluted plot layer (700-1400 KeV) also demonstrates that the MLEM algorithm was able to increase the resolution of peaks with a small number of counts and a higher continuum.

Table 6.3 presents the calculated resolution parameters for three major peaks at different energies. The table shows how the energy resolution for the three peaks greatly improves with deconvolution.

\section{$\underline{6.9 \text { Reactor Experiment and High Enriched } \mathrm{LaBr}_{3}}$ Detector Measurements}

The final goal of this project was to apply a deconvolution protocol to improve highly enriched ATR fuel samples measurements for determination of burnup. However, before committing personnel and monetary resources for a study at the ATR, an experiment was performed in order to determine if the deconvolution of high enriched sample spectra taken with a $\mathrm{LaBr}_{3}$ detector is feasible. In order to test the deconvolution method, highly enriched surrogate fuel samples were used. The sample consisted of ten highly enriched fission wires.

The experiment consisted of irradiating highly enriched fission wires that have the same weight composition of $U^{235}$ that the actual ATR fuel elements do. The irradiation experiment was performed at the AGR reactor facility at ISU. The goal of the experiment was to create a multipeak isotopic fission highly enriched source to test the $1 \mathrm{x} 1 \mathrm{LaBr}_{3}$ scintillator deconvolution protocol. The AGN-201 is a graphite-moderated and selfcontained user facility mainly for training reactor operators and for student projects. The 
reactor has a maximum power level of 5 watts. The reactor is composed of a cylindrical core enclosed in a $20 \mathrm{~cm}$ thick graphite reflector which itself is surrounded by a $10 \mathrm{~cm}$ thick lead shield, followed by a $55 \mathrm{~cm}$ thick water shield.

In order to create the multipeak isotopic fission source, the ten highly enriched wires were bundled and placed inside a double encapsulation system to assure containment of the fission products. The first containment was a polyethylene capsule and the second containment was an aluminum seal casing. The mass composition of the ten wires is $90 \%$ aluminum with approximately $10 \%$ uranium. The uranium that is contained in the wires is $93 \%{ }^{235} \mathrm{U}$ enriched. Once the wires were placed in a double containment system, the capsule was attached to an aluminum rod that slides inside the experiment port during the irradiation. The highly enriched flux wires were irradiated at a maximum power of 4.6 watts with a flux of approximately $2.3 \times 10^{8} \mathrm{n} / \mathrm{cm}^{2} \mathrm{~s}$ for two hours. After the sample irradiation was completed, the capsule was taken out of the reactor and let sit for 18 minutes before being transported to the radiation laboratory.

At the conclusion of the irradiation, the polyethylene container was removed and the aluminum capsule sample was transported to the measurement laboratory. The sample inside the aluminum containment was placed in front of the bismuth collimator inside the lead shield. The sample was positioned $10.1 \mathrm{~cm}$ from the $\mathrm{LaBr}_{3}$ scintillator aluminum housing. Nine spectra of the fission products sample were taken at different decay times.

\subsection{MLEM High Enriched Samples Results}

After the experiment was completed and different highly enriched data were collected, the deconvolution algorithm protocol was tested using high enriched multi- 
peak isotopic fission spectra. Fig. 6.10 compares the convoluted and deconvoluted spectra for a sample taken 48 hours after discharge. The experimental data collected were smoothed to minimize the contribution that noisy data can have creating small artificial peaks. The filter used was a seven point frame quadratic Savitzky-Golay filter. Following the smoothing of the experimental data, the spectra was deconvoluted using the MLEM algorithm implemented in Matlab.

It can be seen from Fig. 6.10 that the MLEM method was able to deconvolute the multi-isotope high enriched data. Again as with the previous deconvolve samples, the method showed that it can be very effective for increasing the individual photo-peak resolution. Table 6.4 shows the improvement in resolution for some of the major peaks shown in Figure 6.10.

In addition, with increasing the resolution of the photo-peaks, the MLEM method was successful in resolving close-lying peaks. The method was able to resolve three pairs of near lying pair peaks 228-250, 531-560, and 743-772 KeV. The close-lying- pair peaks 531 and $560 \mathrm{KeV}$ in the convoluted plot were not even distinguishable from each other. In the convoluted plot, it appears that only the ${ }^{133} \mathrm{I}(529 \mathrm{KeV})$ photo-peak is present; however, in the deconvoluted plot the ${ }^{143} \mathrm{Ce}(560 \mathrm{KeV})$ photo-peak can also be clearly identified. The near lying pair peaks at 743 and $772 \mathrm{KeV}$ are also hard to identify in the convoluted plot; however, they are better resolved in the deconvoluted figure. Fig. 6.10 also shows that there are some small artificial peaks with a very small number of counts created by the deconvolution method. The small perturbations can be attributed to two main factors: the discrepancies between the simulated and true response function as well 
as noise in the data. The small perturbations are also a cost of the effectiveness that the MLEM method have in deconvoluting even the smallest photo-peaks.

The MLEM is a very powerful method; however, having noise in the convoluted spectra can cause the MLEM technique to treat the noise as if small Poisson distribution peaks were present, causing the creation of some nonexistent small perturbations during the deconvolution process. Using a data filter decreases the creation of small peaks; however, smoothing the data too much can affect the counts of the existent photo-peaks. In order to avoid small peak creation, a better resolution data set (more data points) has to be collected as well as finding or developing filtering data techniques better suited for this applications. However, it has to be taken into account that having a better resolution data set will aid in avoiding the creation of artificial peaks by noise in the data; however, it will also increase the number of simulations that are needed to calculate the response function of the detector system. The calculation of the response function requires of one simulation per every data channel that is taken. For this study, 1024 channel data points were collected; therefore, the same number of simulations were needed to calculate the response function of the system. Each simulation for every mono-energetic source during this study was performed using $1 \times 10^{10}$ particles in 32 processors. Also, the increase in channels will increase the time that the iterative MLEM method takes to converge.

The second issue that can cause small perturbations is the discrepancy between the true response function and the response function obtained by simulating an experiment. Obtaining the response function using a Monte Carlo simulations is regarded as the most accurate method to obtain a response function ${ }^{1}$; however, there are always inconsistencies between reality and the modeling of a system; these discrepancies 
can be caused by geometry inaccuracies and assumptions as well as error in the modeling methods.

\section{$\underline{6.11 \text { Conclusion }}$}

A deconvolution protocol was successfully tested for experimental data taken with a $1 \times 1 \mathrm{LaBr}_{3}$ detector. The protocol was tested using one and multi-isotope sources as well as with a highly enriched source. The highly enriched source that was used to test the $1 \times 1 \mathrm{LaBr}_{3}$ deconvolution protocol was created using fission wires irradiated in a nuclear reactor. The protocol was capable of enhancing the resolution of experimental spectra photo-peaks as well as resolving close-lying peaks for the multi-isotopes source and highly enriched sources. For the highly enriched source, the method also proves that it can be used as a tool to aid in the identification of radioisotopes for fission sources. The improvement in quality of the data taken using a $1 \times 1 \mathrm{LaBr}_{3}$ detector due to deconvolution will allow for the design of a rugged and low-maintenance permanent scanning system for the ATR canal.

Finally, the study showed that deconvolution is a powerful tool to enhance the quality of data in order to extract more information from spectra taken with a lower resolution scintillator. However, it also has to be taken into account that the method has a tendency to create small count peaks, in addition to being time- and computer-resources expensive, especially when the accuracy of the method wants to be increased. 


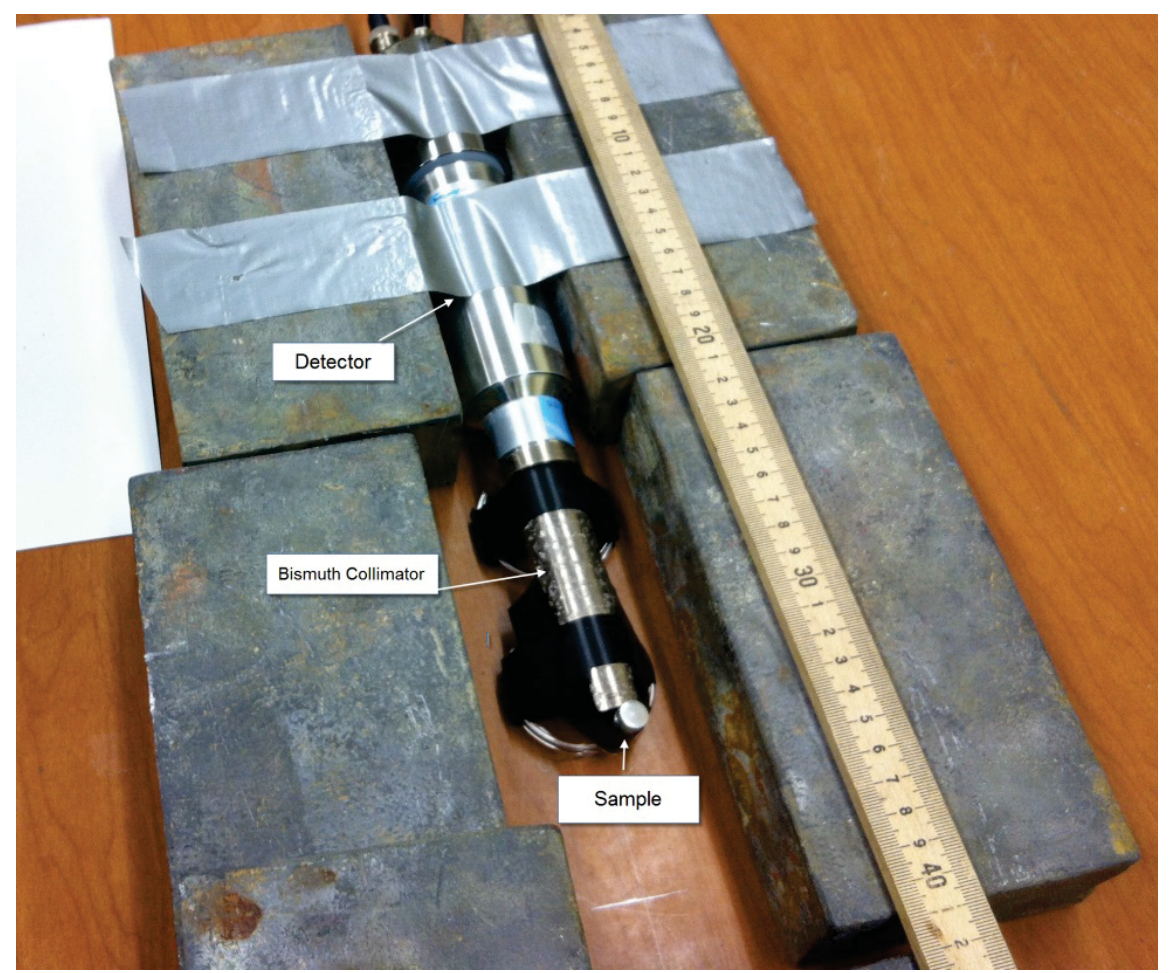

Figure 6.1 Open shield experimental setup

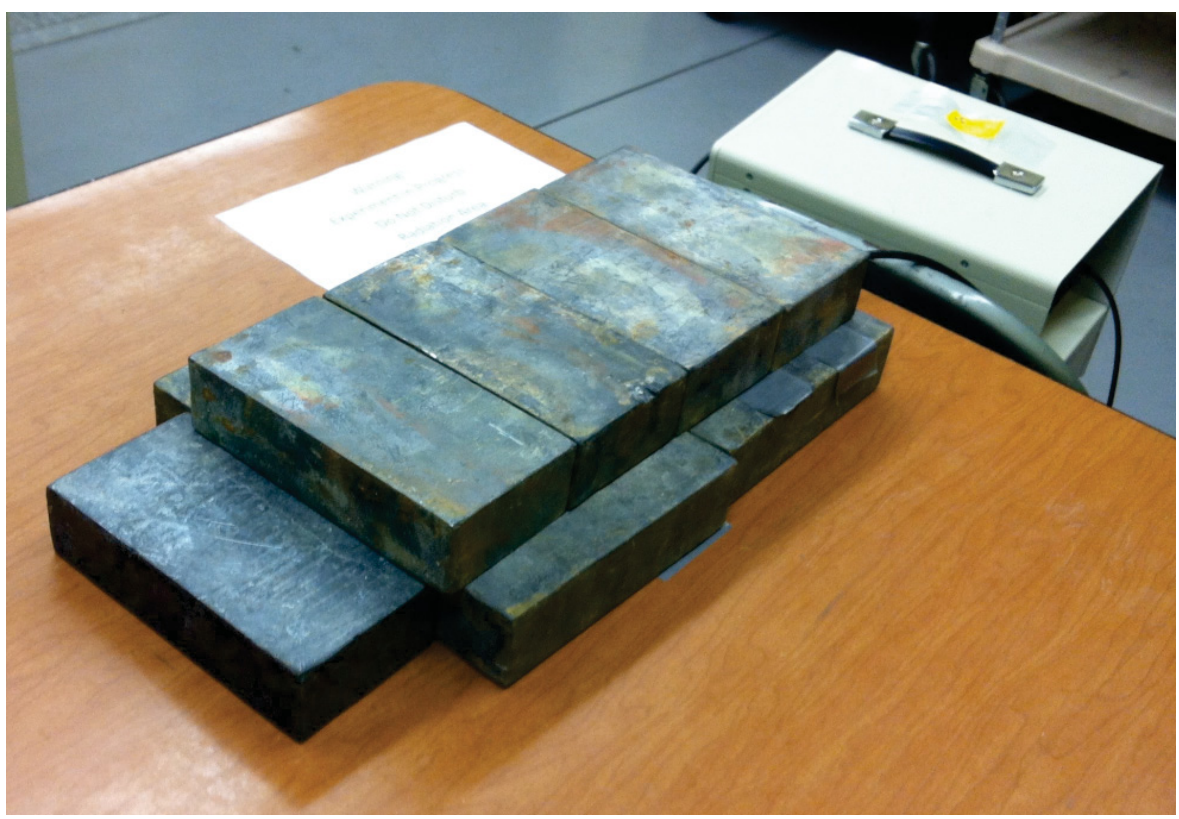

Figure 6.2 Closed shield experimental setup 
Table 6.1.Gamma-energy sources used for Gaussian energy broadening parameters

\begin{tabular}{|c|c|}
\hline Centroid $(\mathbf{K e V})$ & FWHM (KeV) \\
\hline 86.15 & 9.23 \\
\hline 105.78 & 9.22 \\
\hline 123.25 & 9.22 \\
\hline 246.88 & 14.06 \\
\hline 344.94 & 17.06 \\
\hline 592.06 & 24.17 \\
\hline 661.10 & 24.97 \\
\hline 778.35 & 28.05 \\
\hline 872.78 & 35.85 \\
\hline 1102.66 & 50.44 \\
\hline 1173.40 & 35.01 \\
\hline 1332.53 & 40.45 \\
\hline
\end{tabular}




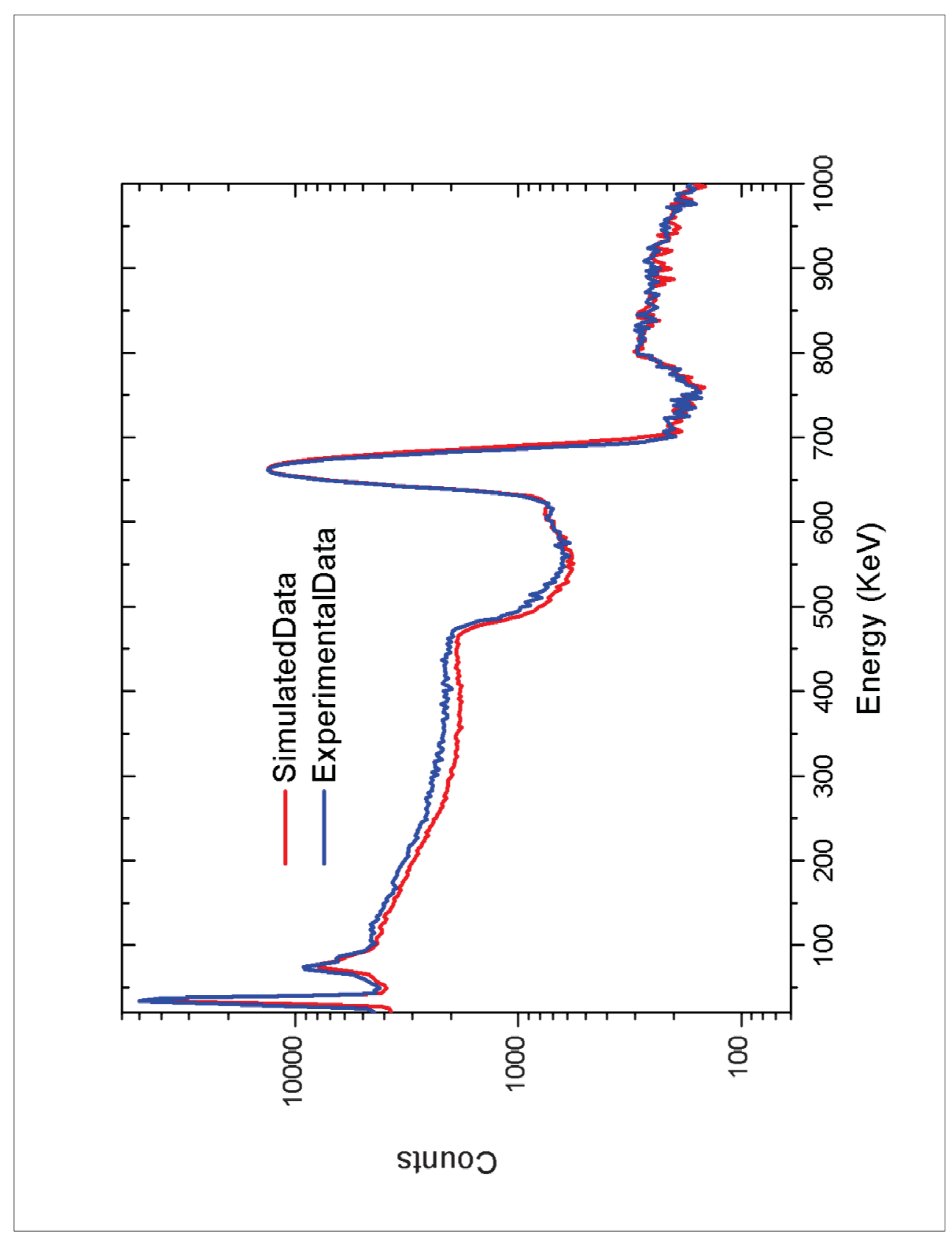

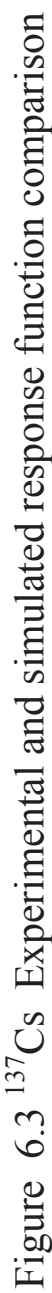




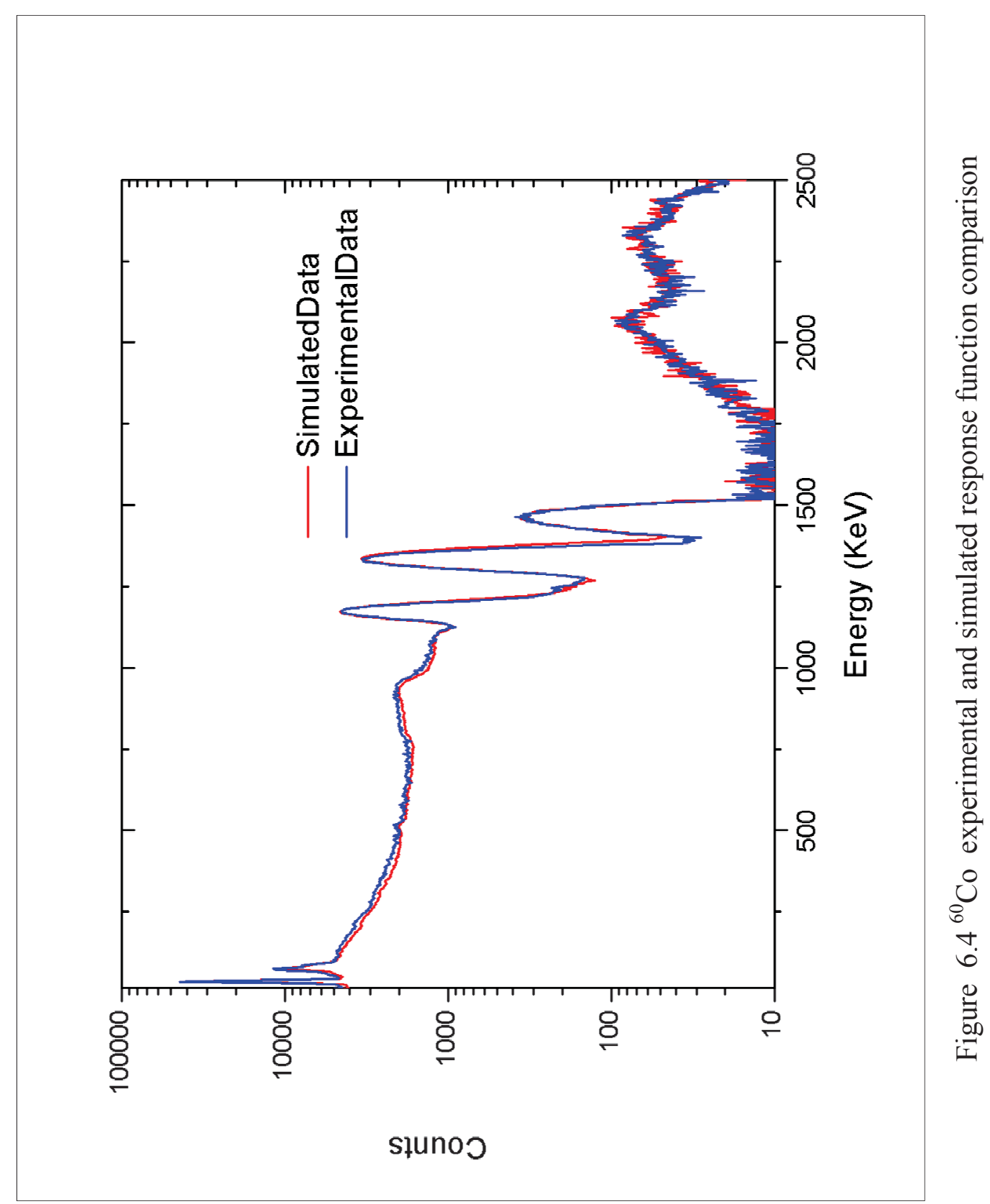




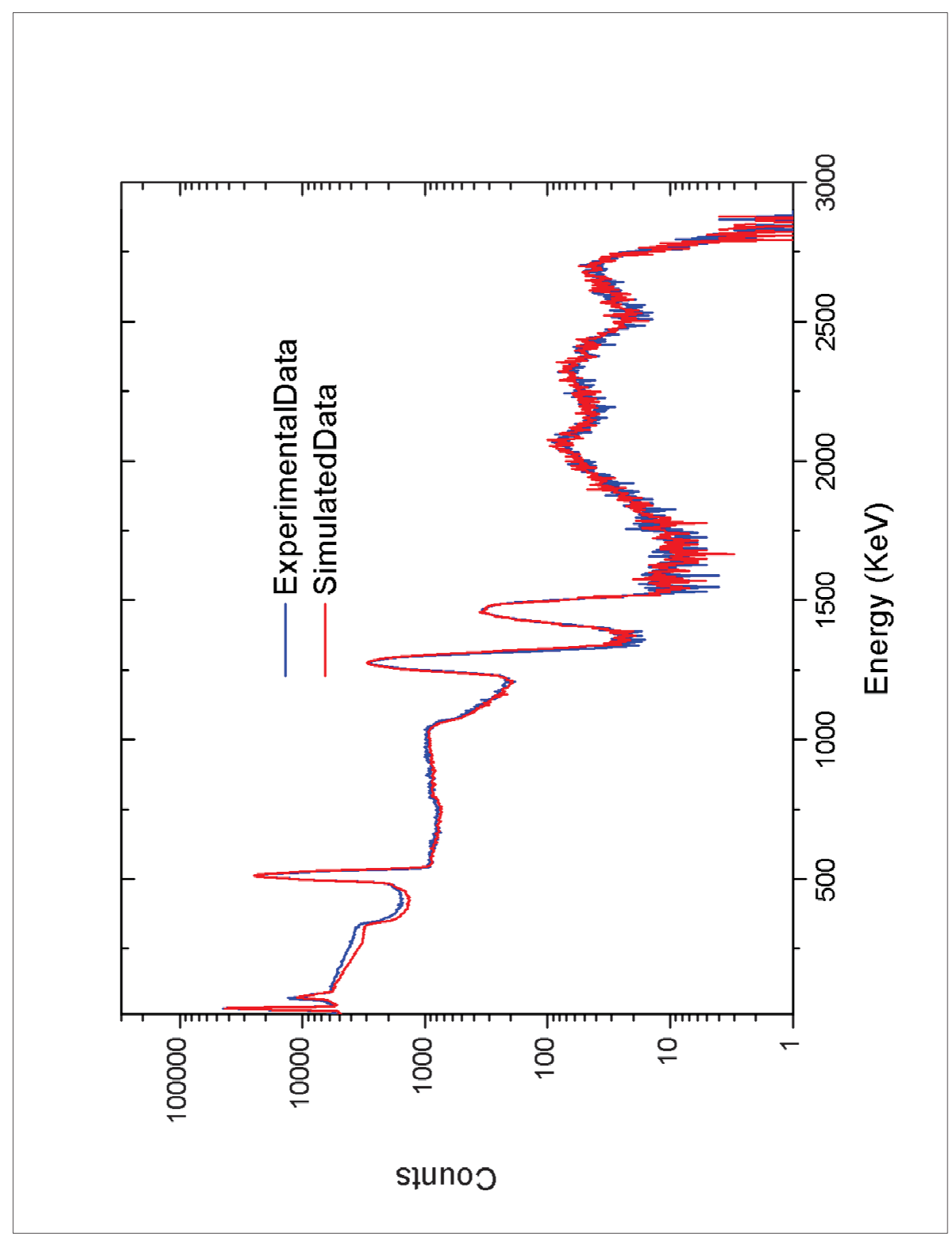

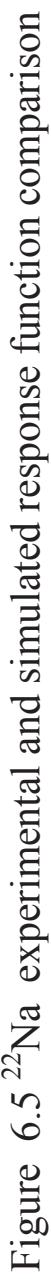




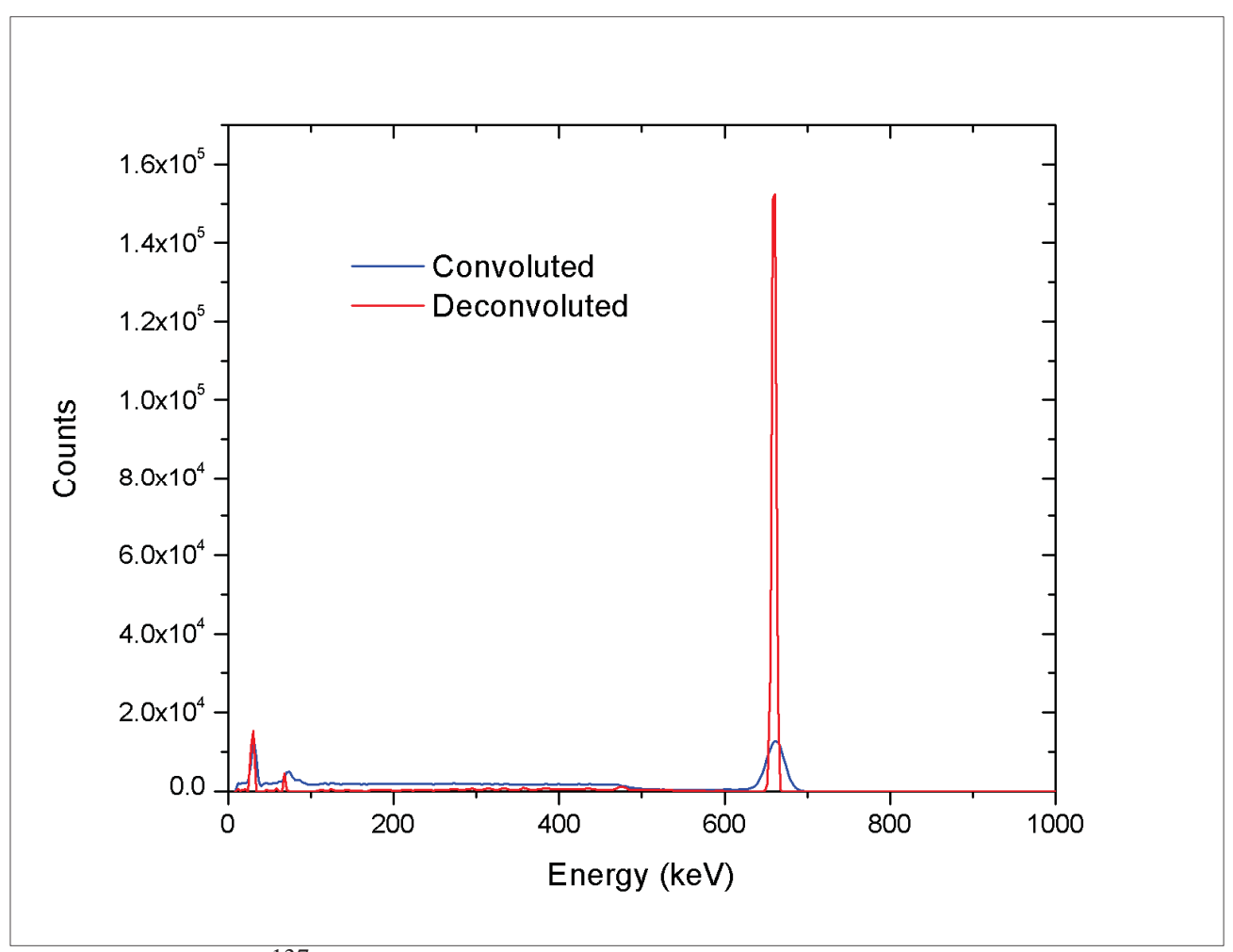

Figure $6.6{ }^{137} \mathrm{Cs}$ convoluted and deconvoluted spectra comparison

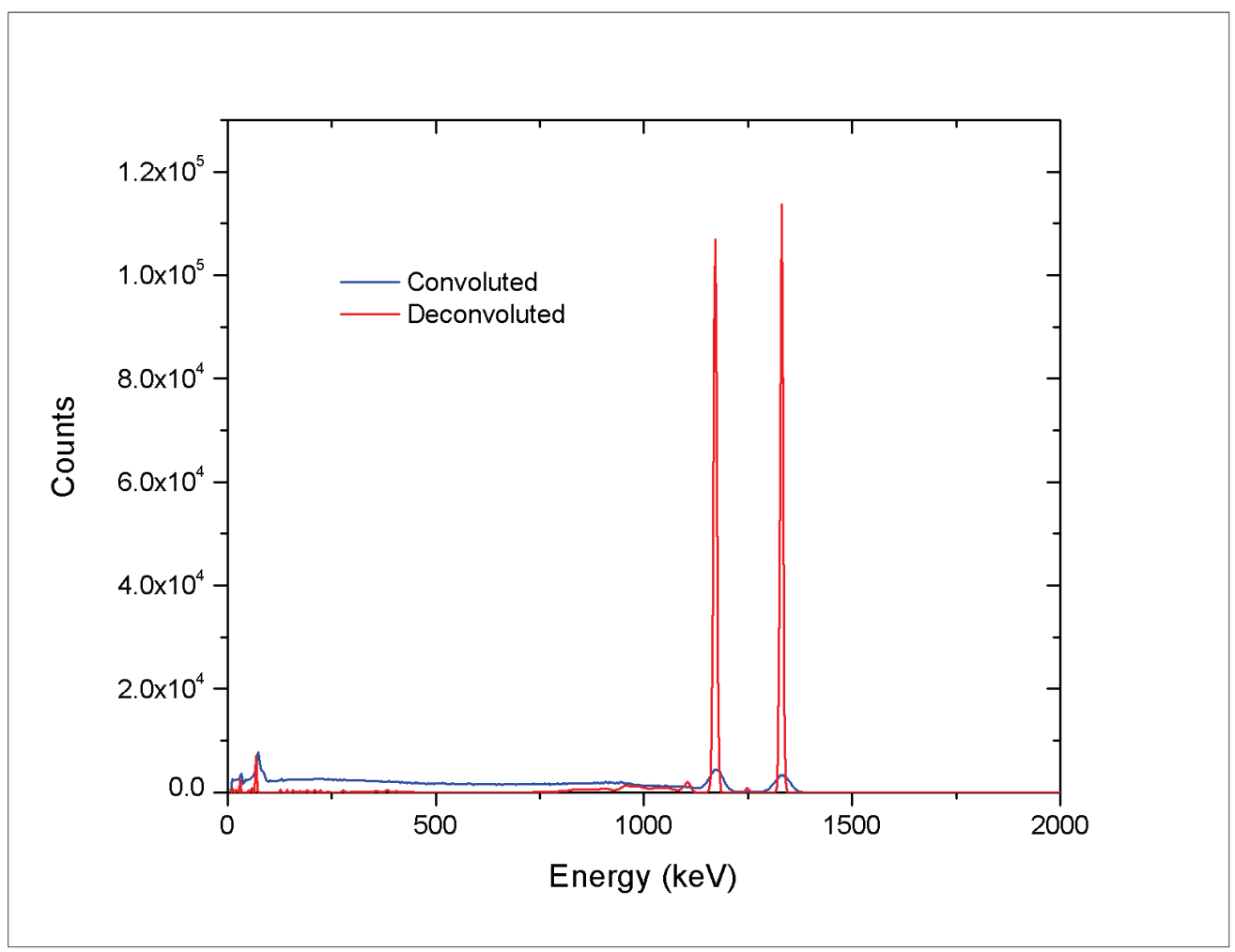

Figure $6.7{ }^{60}$ Co convoluted and deconvoluted spectra comparison 


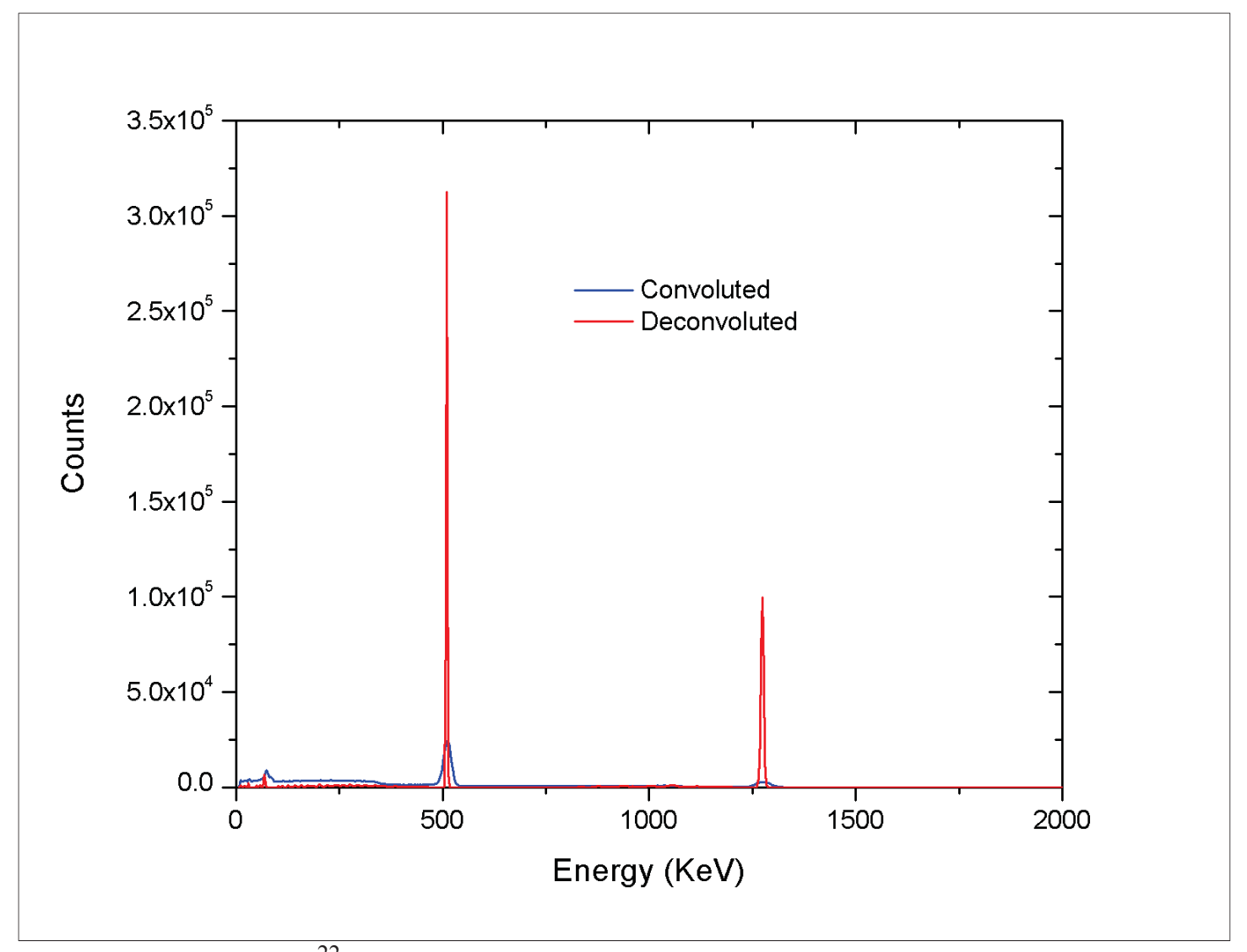

Figure $6.8^{22}$ Na convoluted and deconvoluted spectra comparison.

Table 6.2 Resolution and performance parameters comparison

\begin{tabular}{|c|c|c|c|c|c|}
\hline Source & $\begin{array}{c}\text { Peak } \\
\text { Energy } \\
(\mathrm{KeV})\end{array}$ & Spectrum & $\begin{array}{c}\text { Peak } \\
\text { Resolution } \\
(\%)\end{array}$ & Peak/Compton & $\begin{array}{l}\text { Peak } \\
\text { /Total }\end{array}$ \\
\hline \multirow[b]{2}{*}{$\mathrm{Cs}^{137}$} & \multirow[b]{2}{*}{662} & $\begin{array}{c}\text { Experimental } \\
\text { Spectrum }\end{array}$ & 3.873 & 7.447 & 1.024 \\
\hline & & Deconvolution & 0.946 & 399.041 & 2.885 \\
\hline \multirow[b]{4}{*}{$\mathrm{Co}^{60}$} & \multirow[b]{2}{*}{1173} & $\begin{array}{c}\text { Experimental } \\
\text { Spectrum }\end{array}$ & 3.051 & 1.903 & 0.207 \\
\hline & & Deconvolution & 0.798 & 255.489 & 1.267 \\
\hline & \multirow[b]{2}{*}{1332} & $\begin{array}{c}\text { Experimental } \\
\text { Spectrum }\end{array}$ & 3.106 & 3.341 & 0.215 \\
\hline & & Deconvolution & 0.632 & 120.472 & 1.326 \\
\hline \multirow[b]{2}{*}{$\mathbf{N a}^{22}$} & \multirow[b]{2}{*}{662} & $\begin{array}{c}\text { Experimental } \\
\text { Spectrum }\end{array}$ & 3.111 & 3.727 & 0.176 \\
\hline & & Deconvolution & 0.607 & 319.310 & 1.034 \\
\hline
\end{tabular}




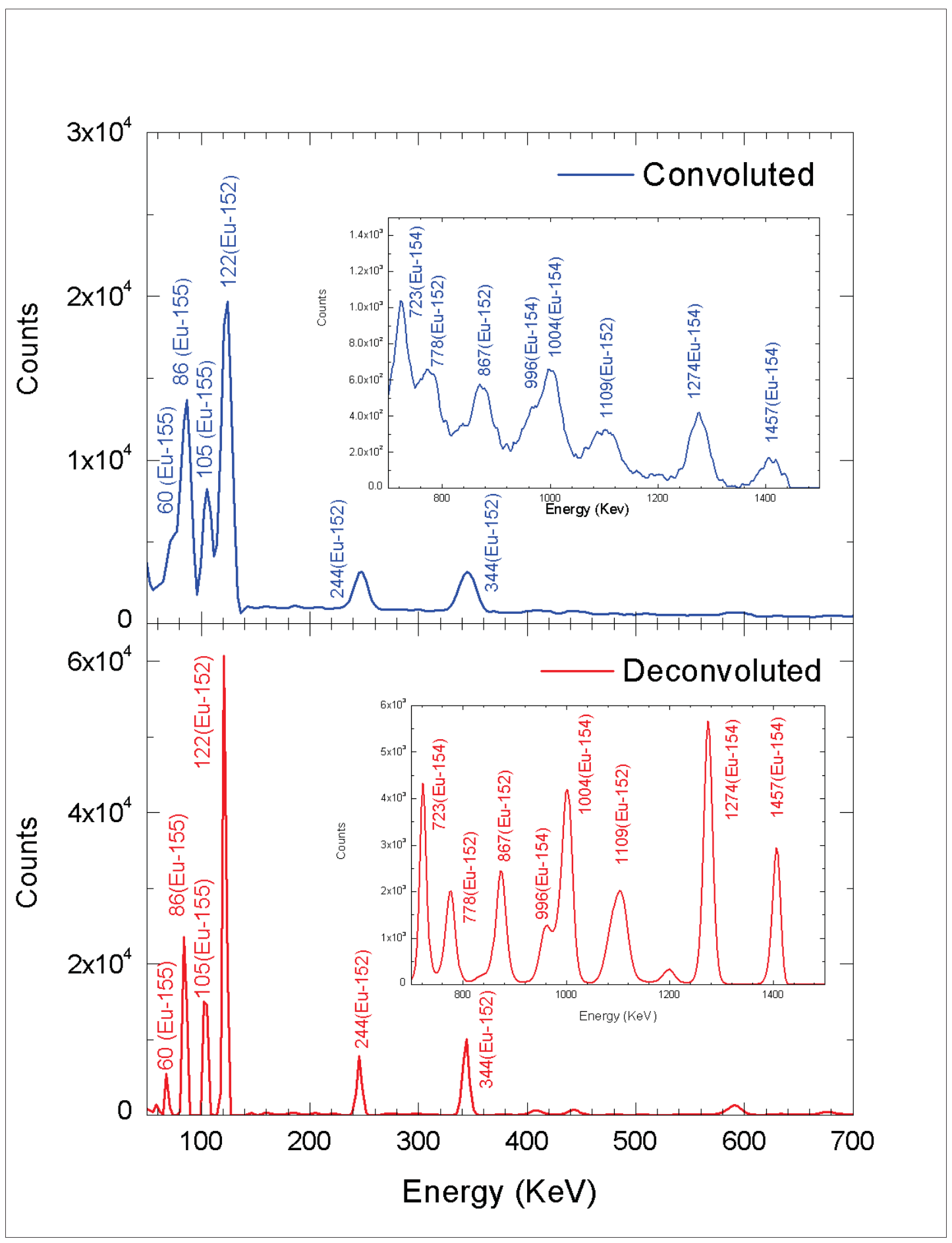

Figure 6.9 Multi-isotope europium source convoluted and deconvoluted comparison 
Table 6.3 Resolution multi-isotope source comparison

\begin{tabular}{|c|c|c|c|}
\hline \multirow{4}{*}{ Source } & $\begin{array}{c}\text { Peak } \\
\text { Energy } \\
(\mathbf{K e V})\end{array}$ & Spectrum & $\begin{array}{c}\text { Peak } \\
\text { Resolution } \\
(\mathbf{\%})\end{array}$ \\
\hline \multirow{3}{*}{$\mathbf{E u}^{\mathbf{1 5 2 , 1 5 4 , 1 5 5}}$} & $\mathbf{1 2 2}$ & $\begin{array}{c}\text { Experimental } \\
\text { Spectrum }\end{array}$ & 8.386 \\
\cline { 2 - 4 } & & Deconvolution & 3.731 \\
\cline { 2 - 4 } & $\mathbf{2 4 4}$ & $\begin{array}{c}\text { Experimental } \\
\text { Spectrum }\end{array}$ & 5.681 \\
\cline { 2 - 4 } & & Deconvolution & 1.740 \\
\cline { 2 - 4 } & \multirow{2}{*}{$\mathbf{1 2 7 4}$} & $\begin{array}{c}\text { Experimental } \\
\text { Spectrum }\end{array}$ & 3.016 \\
\cline { 2 - 4 } & & Deconvolution & 1.564 \\
\hline
\end{tabular}




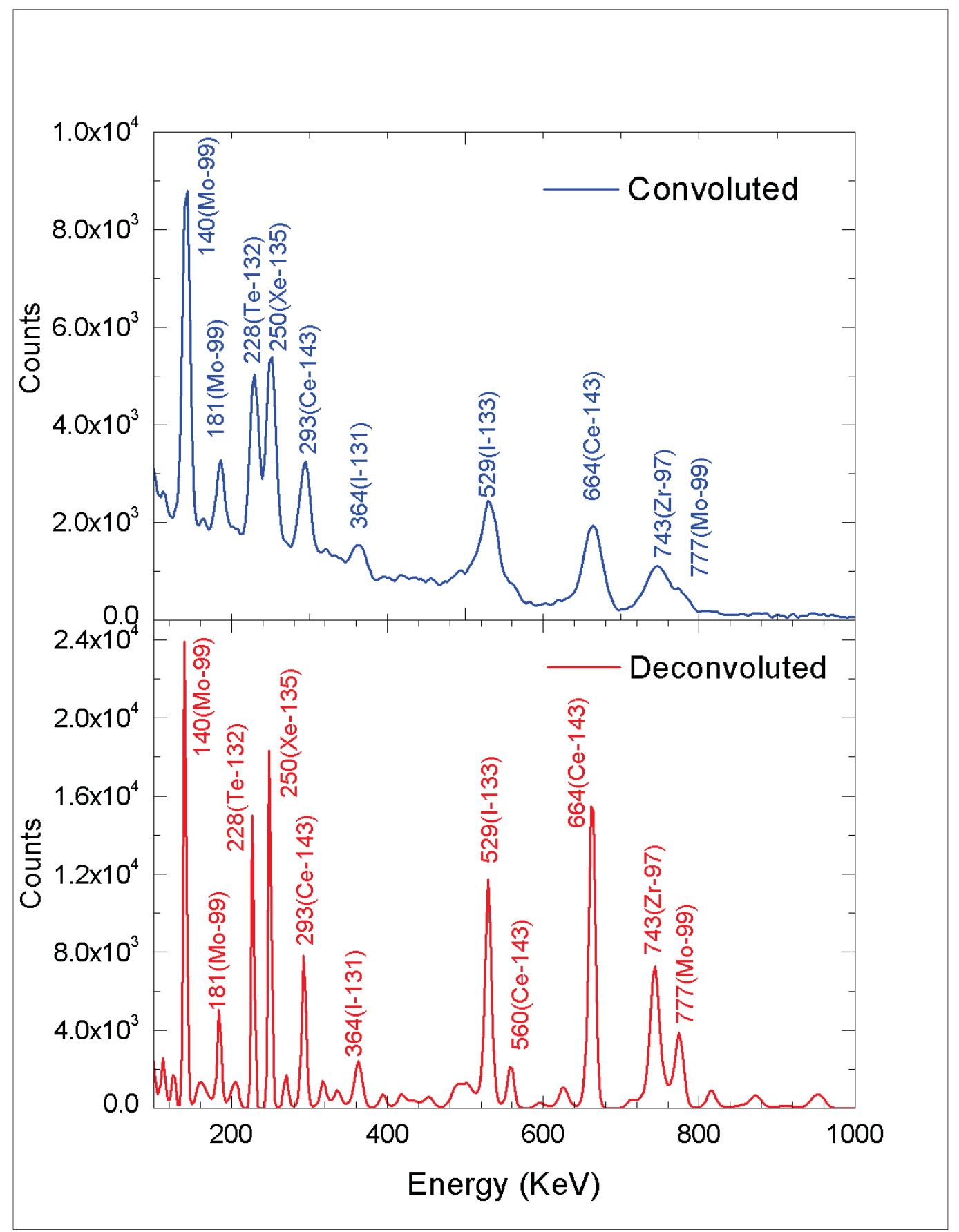

Figure 6.10 Highly enriched fission products source convoluted and deconvoluted comparison 
Table 6.4 Fission product multi-isotope source resolution comparison

\begin{tabular}{|c|c|c|c|}
\hline \multirow{3}{*}{$\mathbf{M o}^{\mathbf{9 9}}$} & $\begin{array}{c}\text { Peak Energy } \\
(\mathbf{K e V})\end{array}$ & Spectrum & $\begin{array}{c}\text { Peak Resolution } \\
\mathbf{( \% )}\end{array}$ \\
\hline \multirow{2}{*}{$\mathbf{X e}^{\mathbf{2 5 0}}$} & $\mathbf{1 4 0}$ & Experimental Spectrum & 8.114 \\
\cline { 3 - 4 } & \multirow{2}{*}{$\mathbf{2 5 0}$} & Deconvolution & 4.017 \\
\hline \multirow{2}{*}{$\mathbf{C e}^{\mathbf{1 4 3}}$} & & Experimental Spectrum & 4.451 \\
\cline { 3 - 4 } & $\mathbf{6 6 4}$ & Deconvolution & 2.887 \\
\cline { 3 - 4 } & & Deconvolution & 3.909 \\
\hline
\end{tabular}




\section{$\underline{6.12 \text { References }}$}

1 Meng, L.J.; Ramsden, D. An Inter-comparison of Three Spectral-Deconvolution Algorithms for Gamma-ray Spectroscopy, IEEE Transactions on Nuclear Science, 2000,Vol. 47, No. 4, 1329-1336.

2 Navarro, J.; Aryaeinejad, R.; Nigg, D.W. A Feasibility Study to Determine Cooling Time and Burnup of Advanced Test Reactor Fuel Using a Nondestructive Technique and Three Types of Gamma-ray Detectors. Journal of ASTM International, 2012, Vol. 9, Issue 3.

3 Maximum-Likelihood Expectation-Maximization (ML-EM) Iterative Reconstruction Algorithm,class notes, UC Berkley. Retrieve from http://inst.nuc.berkeley.edu/NE107/Lectures/MLEM.pdf

4 X-5 Monte Carlo Team, April 2003A general Monte Carlo N-Particle Transport Code, Version 5, Overview and Theory, Vol. 1, pp. 106-107, Los Alamos, NM.

5 Pelowitz, D. B. MCNPX A General N-Particle Transport Code, version 2.6.0". LACP-07-1473, Los Alamos, NM.

6 Shepp, L.A.; Vardi, Y.; Maximum Likelihood Reconstruction for Emission Tomography. IEEE Trans. Medical Imaging, 1982, Vol 1(2), 113-122.

7 Navarro, J.; Ring, T. A.; Nigg, D.W. Gamma-Ray Spectra Deconvolution of a $\mathrm{LaBr}_{3}$ 1" x 1" Scintillator for Nondestructive ATR Fuel Burnup On-Site Predictions 2013. (In process of being submitted). 


\section{CHAPTER 7}

\section{PERMANENT ATR FUEL SCANNING SYSTEM \\ PRELIMINARY DESIGN}

After performing the feasibility and optimization study, a preliminary design of the permanent system was created. The preliminary design was performed based on the results obtained in this thesis. The ATR Fuel Burnup Measurement System (FBUMS) will consist of four major subsystems: $\mathrm{X}$ and $\mathrm{Z}$ scan system axis, lead screw trolley, detector housing, two collimator pipes, fuel element carriage system, and a computer control system. The default setting of the detection system will be above the water; however, the capability of going underwater will also be available.

The system shown in Fig. 7.1 will be capable of moving the detection system and placing it exactly at a desired spot with accuracy of $1 / 1000 "$. The tower structure will be placed on the east side of the canal. The system will include parallel rods to hold the detection system above the water and a U-shape structural frame in the water with the Fuel Element Carriage (FEC) at the bottom to hold a fuel assembly. Measurements will be performed mainly with the detector above the water and with the 14 ' collimated pipe attached to it; however, the capability of performing measurements underwater will also be available. 
Motors to move the detection system in "X" and "Z" directions will be available in the system. The data analyzed showed that the housing collimator developed for the feasibility can be utilized for the permanent system. FBUMS will be configured as a two axis (X-Z) computer controlled scanning system. Two parallel 3" diameter stainless steel shafts will form the $\mathrm{X}$ axis which spans the width of the ATR canal. Each end is mounted on a set of wheeled trucks with locks that will retain the FBUMS in position over the canal. A Lead Screw Trolley (LST) will move the detector system along the $\mathrm{X}$ axis and a secondary trolley will move the system in the $\mathrm{Z}$ direction when necessary. The trolley, positioned between the shafts, will be incrementally driven back and forth. This movement is parallel and directly above the length of the fuel element suspended. The $\mathrm{X}$ axis provides a trolley parking area at one end to accommodate fuel element loading in the Fuel Element Carriage (FEC) below. The ATR fuel element to be inspected will be supported horizontally in the FEC suspended approximately 14 feet below the canal water level. The FEC will automatically position and center the fuel element in the same position relative to the detector before every inspection.

The Detector Housing Assembly (DHA) will be mounted on two short horizontal slides on the X Scan Carriage (XSC) for manual positioning of the assembly over either Down Pipe Assembly (DPA). The DPAs will be approximately 14 feet long, air filled pipes with different sized bismuth collimators located at the bottom end of the tubes. Shielding on the DPA will insure that both down pipes are covered at all times to prevent streaming radiation out of the pool during inspection of the fuel elements, especially for the pipe that is not being used. 
The $\mathrm{Z}$ axis lifts the XSC, DHA, and DPAs vertically, a maximum of 24 inches to increase the distance between the end of the pipe and consequently the system detector and the fuel element under inspection. A computer system will control the mechanical operation of the scanning system and will also collect the spectral data from the detector system. This system also allows us to do measurements along the fuel element to obtain the radiation profile for investigating the fuel burnup uniformity. A successful design of the permanent system will allow for collection of higher quality of data allowing better confidence in burnup and cooling time calibration as well as having an on-site fuel tool to aid with ATR fuel management.

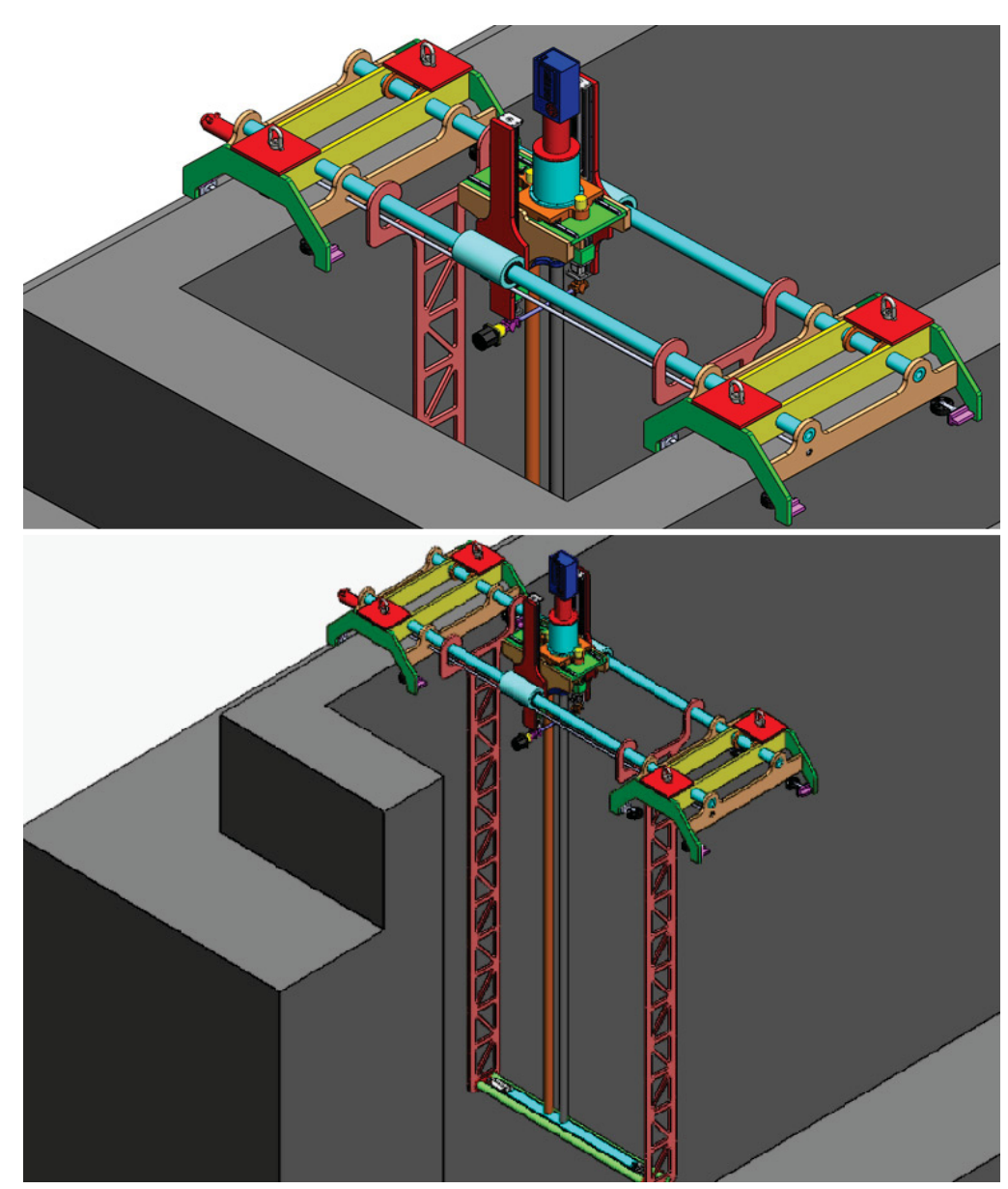

Figure 7.1 Conceptual ATR fuel burnup measurement system 


\section{CHAPTER 8}

\section{SUMMARY, CONCLUSION, AND FUTURE WORK}

\subsection{Summary and Conclusions}

The results shown during this study prove that it is feasible to obtain a meaningful gamma-ray signal from the ATR canal using three different kinds of detectors. The signal collected using the HPGe detector has superior photo-peak resolution, the close-lying peaks in the spectra are easy to identify, and the data extracted with these detectors provide the most isotopic information out of the fuel elements. The signal collected using the $\mathrm{LaBr}_{3}$ detectors showed good photo-peak resolution along with providing isotopic information of major photo-peaks; however, for peaks with small number of counts as well as for close-lying peaks, the spectra collected using these detectors lacks proper resolution.

$\mathrm{LaBr}_{3}$ detectors need very low maintenance and are a great fit for in-situ measurements as well as being able to perform underwater measurements. The information extracted using the HPXe provided far less isotopic information than the other two detectors; however, the HPXe detector can withstand long-term radiation without losing stability and can be placed very close to the ATR fuel elements $(>3$ inches) while still collecting meaningful data. 
The study also showed that the above the water configuration can extract the most information out of the fuel elements. The study also proved that an underwater capability to extract gamma-ray spectra can be available at the ATR canal for fuel experiments and material analysis. The ATR canal feasibility gamma measurements establish that in order to have a versatile gamma scanning system, each one of the detectors tested during the study can be used for different tasks.

In addition, the data collected with the three detectors were used to create a method to predict burnup nondestructively for ATR fuel elements. The study demonstrate that predicting burnup using gamma spectroscopy data can be accomplished at the ATR canal on-site using data collected with three different kinds detectors. The study also showed that two isotopic peak area ratios $\left({ }^{134} \mathrm{Cs} /{ }^{137} \mathrm{Csand}\right.$ $\left.{ }^{134} \mathrm{Cs} /{ }^{144} \mathrm{Ce}\right)$ and two absolute peak areas $\left({ }^{134} \mathrm{Cs}\right.$ and $\left.{ }^{137} \mathrm{Cs}\right)$ consistently have a linear relationship with ATR burnup fuel. The ${ }^{134} \mathrm{Cs} /{ }^{144} \mathrm{Ce}$ ratio shows a linear relationship with burnup. This has not been previously reported as a burnup monitor in any other study. The results also demonstrated that the data extracted from the ATR canal can be used to predict the cooling time of fuel elements. The study found that the ${ }^{144} \mathrm{Ce} /{ }^{137} \mathrm{Cs}$ isotopic peak area ratio as well as ${ }^{144} \mathrm{Ce},{ }^{95} \mathrm{Zr}$, and ${ }^{95} \mathrm{Nb}$ absolute isotopic peak areas can be used as cooling time monitors for ATR fuel. The first stage of the study established that data can be extracted on-site and that the data have the quality to be used as a validation tool as well as to create calibration curves to predict burnup and cooling time of fuel elements on-site.

The ATR canal measurements demonstrate that a scanning system to extract data and predict burnup is feasible. It also gave an insight into what kind of design is 
needed in order for the system to perform well under the conditions at the ATR canal. The decision of which detector can used as a primary detector of the fuel scanning system had to take into account that the apparatus will be placed at the ATR canal and that requires it to have little maintenance. Taking into account that the fuel scanning system will be in an operational facility and not in a laboratory environment, the possibility that the $\mathrm{LaBr}_{3}$ can be better suited for the ATR canal was explored.

The major drawbacks of using the $\mathrm{LaBr}_{3}$ detector as a primary detector of the fuel scanning system were related to the energy photo-peak resolution of the detector and quality of the spectra that contribute to misidentification of peaks and reduce confidence of the calibration curves. It was determined that if these issues could be improved, the $\mathrm{LaBr}_{3}$ scintillator would be a better option than the HPGE spectrometer as a primary detector for the permanent system.

Once it was determined that from an operational standpoint the $\mathrm{LaBr}_{3}$ scintillator was a better option as a primary detector for the fuel scanning system, a deconvolution methodology was developed to improve the spectra resolution. The deconvolution methodology consisted of calculating the response function of the detector, validating that response, and implementing the MLEM algorithm in Matlab. The methodology was initially tested using one- and multi-isotope simulated data. After it was proven that the deconvolution methodology is capable of enhancing photo-peak resolution as well as resolving close-lying peaks, a surrogate source based on ATR fuel elements data was created. Four fuel surrogate simulated elements were created in order analyze how deconvolution affected nondestructive burnup prediction. The fuel data 
from the four simulated elements was deconvoluted and the results showed that deconvolution improves the confidence in burnup calibration predictions.

The final phase of the study was to test the deconvolution methodology developed for the $\mathrm{LaBr}_{3}$ detector with experimental isotopic sources; however, because the ultimate goal is to deconvolute fuel fission spectra, a validation experiment was developed. The experiment consisted of irradiating fission wires that have the same uranium enrichment as the ATR fuel elements. After the experiment, an irradiated source data at different decay stages as well one-isotope and multi-isotope spectra were collected using the 1"x1" $\mathrm{Labr}_{3}$ detector. The spectra collected were then submitted to the deconvolution methodology previously developed. The results showed that for the one-isotope deconvoluted spectra, not only the resolution of the individual photo-peaks was enhanced considerably; it also proved that the peak-to-Compton and peak-to-total ratio performance parameters improved. The results also demonstrated that for the multi-isotope spectrum, deconvolution improves individual photo-peak resolution as well as helping resolve close-lying peaks. Finally, the deconvolution method was applied to the high enriched fission source created. The results established that the individual photo-peak resolution improves with deconvolution as well as establishing that the deconvolution aids isotope identification.

\section{$\underline{8.2 \text { Future Work }}$}

The study developed in this thesis established that a low-maintenance, efficient, and reliable scanning system that is able to extract useful information out of the ATR fuel elements in a nondestructive manner can be developed and designed. The study 
also demonstrated that there is a consistent linear relationship between some ATR fuel spectra ratios and absolute isotopic areas with burnup, and that this relationship can be used to predict burnup on-site. Having the capability to predict burnup on-site with confidence will allow us to improve fuel cycles management operations and could aid in extending the life of the fuel elements. The study also established that a rugged and low-maintenance scintillator can be used as a primary detector of the ATR fuel scanning system if deconvolution is applied successfully.

The study presented in this thesis laid the foundation to design and construct a permanent fuel scanning system; however, some areas have to be developed before the fuel scanning system can be fully implemented as a validation data collection and burnup prediction tool. The first area of development has to be focus on creating techniques to improve the prediction of the response function of the $\mathrm{LaBr}_{3}$. The creation of artificial peaks during the deconvolution process can be attributed to the discrepancies between simulated and experimental response function. The thought today in the gamma spectroscopy deconvolution community is that the discrepancies between experimental and simulated response functions are the price that has to be paid when Monte Carlo tools are used; however, after performing this study, the author differs with this mainstream belief. There are techniques used in other fields that can potentially help to decrease the discrepancies between simulations and experimental response functions. The implementation of those techniques will have to be tested and potentially they will improve the prediction of response function, hence improving the deconvolution process. 
The second area of development consists in establishing a technique to use burnup calibrations to validate reactor physics codes. Burnup validation data today are very scarce due to the fact that the only method to validate burnup is with the use of destructive techniques. Destructive techniques are very hard to implement because it means that fuel elements have to be submitted to chemical assays in order to measure the isotopes present. Destroying fuel elements carries the potential of causing unnecessary exposure to workers, is expensive, and cannot be performed routinely. The linear relationship between burnup and isotopic area ratios or absolute areas has proven to be consistent; however, there is no formal process to validate burnup data using gamma spectra collected from fuel. Developing a methodology to validate burnup calculations using gamma spectroscopy data will allow for the validation of the new generation of reactor codes using a wide variety of fuel elements and not only perform validation with a few data points from a few fuel elements that were destructively assay in the past. The third area of development that is needed is to develop a methodology to investigate how burnup of ATR fuel is affected in different axial and radial directions. The fuel scanning system will allow collecting burnup data axially and radialy. Collecting this information and determining how this information will help the ATR canal operators to better manage the fuel and how the data can be use for validation purposes is an important study that has to be performed. 
APPENDIX A

DISTANCE MEASUREMENT DEVICE SELECTION

FOR THE ATR FUEL SCANING SYSTEM

FINAL DESIGN 
In the previous chapters of this study, it was emphasised the need to know with accuracy the distance measurement between the detector and the fuel target. Validation methods, burnup and cooling time calibrations, as well as deconvolution methods confidence involve knowing the geometry of the system with certainty. Two approaches were tested in order to determine which method was more accurate in determining the distance of the detector and fuel target underwater. The two methods tested were ultrasonic and laser. The measurements performed with both techniques indicated that the laser was more accurate measuring the distance underwater. Besides the laser apparatus more accurately measuring the distance, there was an additional advantage of using this method; the added additional benefit was being able to see the exact location of the laser spot hitting the fuel element. Locating the exact point were the ultrasonic method was measuring was not possible. This is important because the ATR fuel plates are curved and knowing the location of where the detector is pointing is crucial. The laser tested and selected for the permanent system was a small but very accurate AR-700 laser unit from Acuity Company. This is a Class 3B laser. The unit consists of a laser box, a controller box, and a display, as shown in Fig. A.1.

The AR700 is a triangulation sensor that measures distance using a laser beam, miniature cameras, and a microprocessor. Different models can be used for different measurement ranges. They vary in range from 0.1 to 50 inches and the accuracy is generally $0.1 \%$ with linearity of around $0.03 \%$. A variety of configuration settings can be selected via serial port or by using the function button and function display LEDs. The sample rate can be specified and the sensor has capability above 9400 samples per seconds. Sampling can be turned on and off and can be triggered using an input signal 
or a serial port commands. Measurement outputs can be in the form of serial data (RS232), Analog output, and Limit outputs using two switches. Fig. A.2 shows the laser unit inside the waterproof housing. The laser beam is projected from the red circle's aperture at the bottom on a target surface, where it is focused to a small spot. From there, the laser light is scattered in all directions. These scattered beams are collected by three camera lenses located on the top window. The position of the laser spots imaged in these lenses is then processed to measure the distance.

Distance calibrations were performed in the air as well as in the water. Fig. A.3 and A.4 show the two setup configurations. A dummy fuel element made of aluminum to resemble the actual ATR fuel element is used in our measurements. The laser system was mounted on a 3 " pipe so that the laser beam and the center axis of the pipe are parallel and 2.5" apart. This means the detector is viewing a spot on the fuel element that is along the length of the fuel element 2.5" from the laser spot. Both measurements in the air and water were determined to be within accuracy of 0.1 inches. 


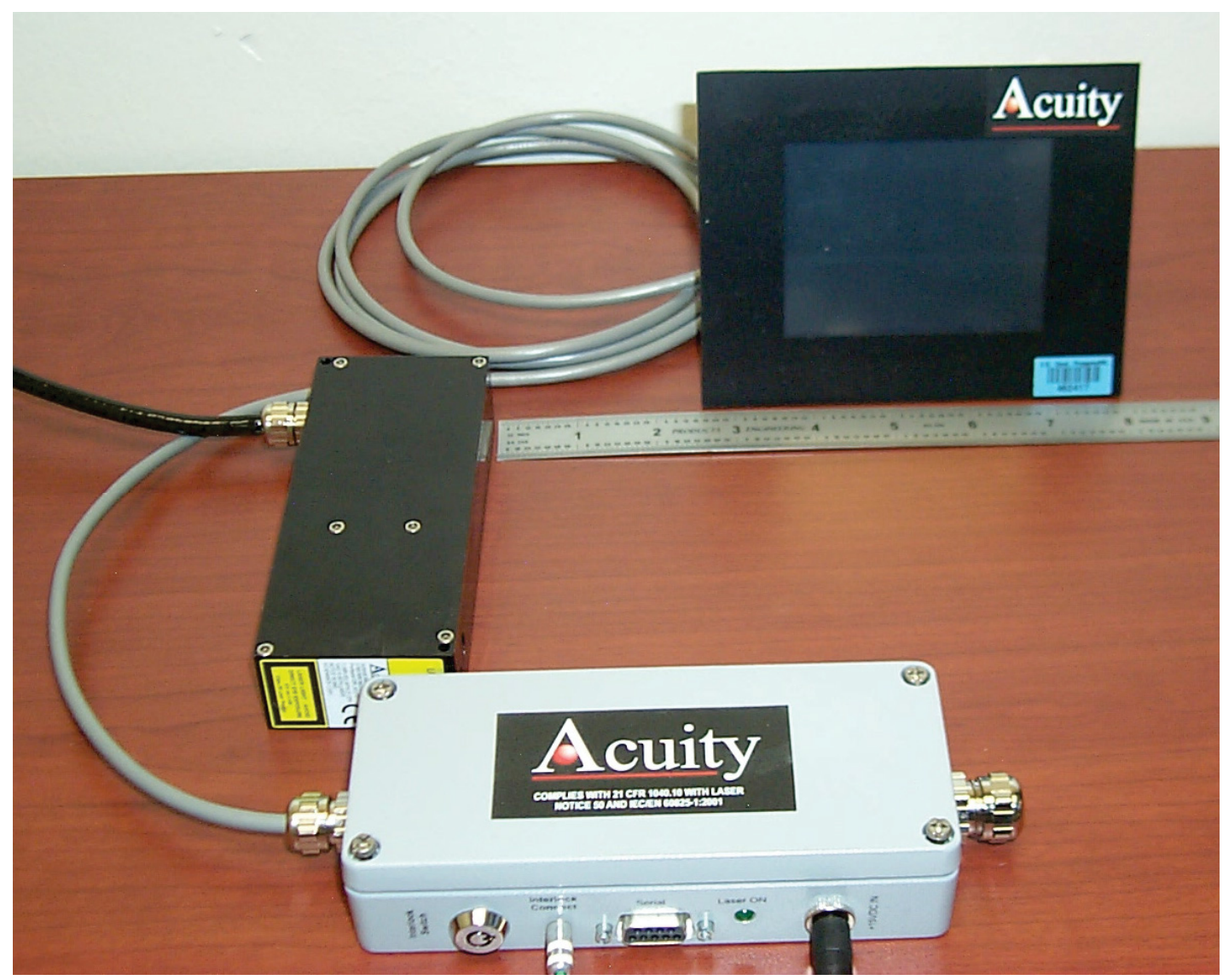

Figure A.1 Laser unit with a controller box and a display. 


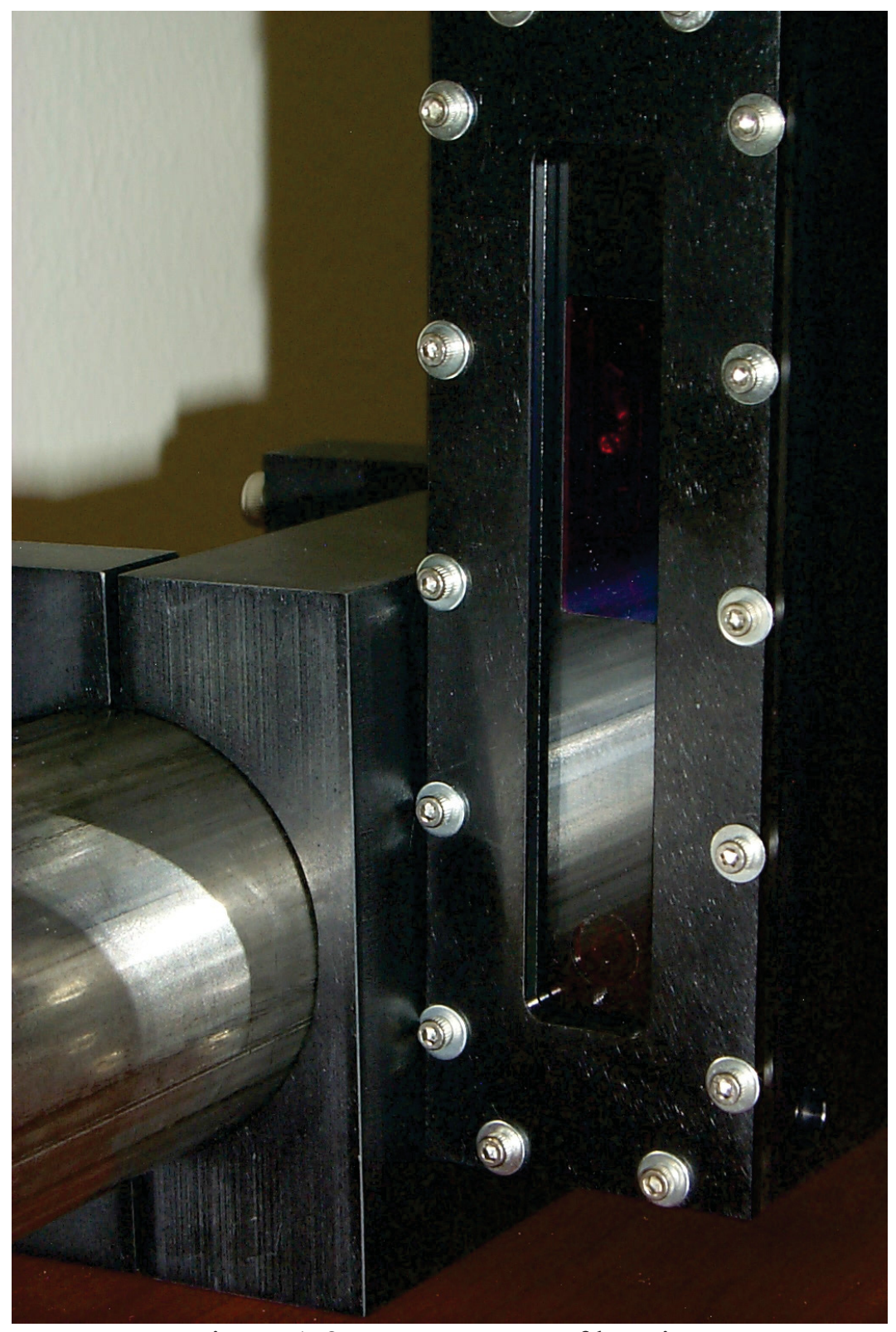

Figure A.2 Laser waterproof housing 


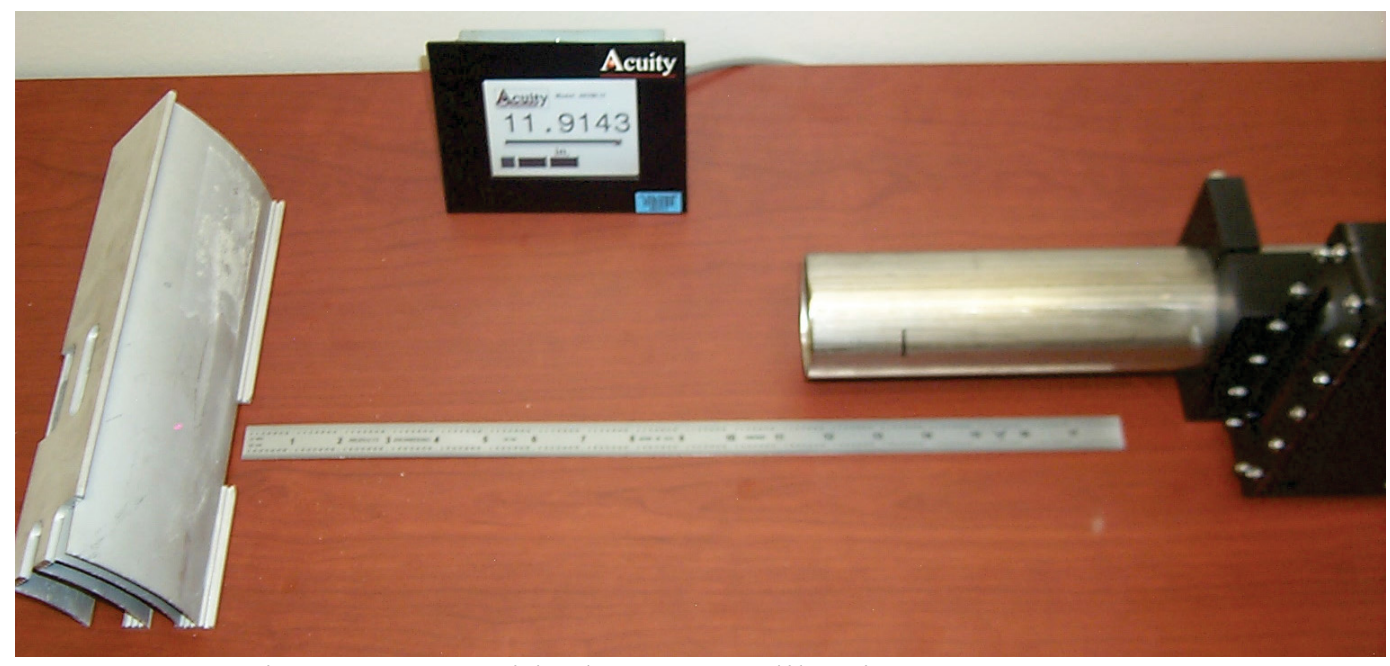

Figure A.3 Outside the water calibration measurements

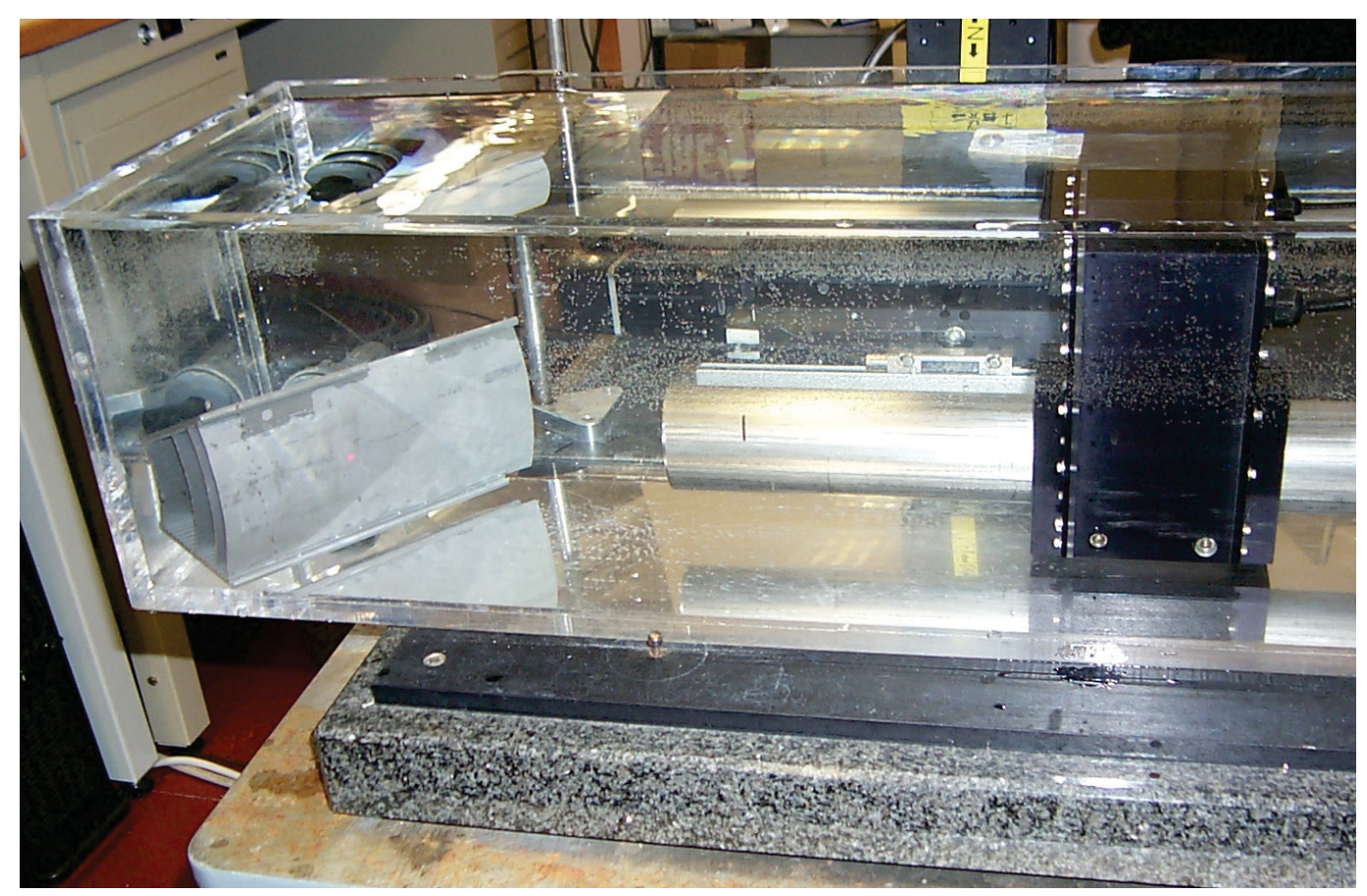

Figure A.4 Underwater calibration measurements 
APPENDIX B

MLEM EXAMPLE MATLAB CODE 


\section{Matlab Code MLEM Example}

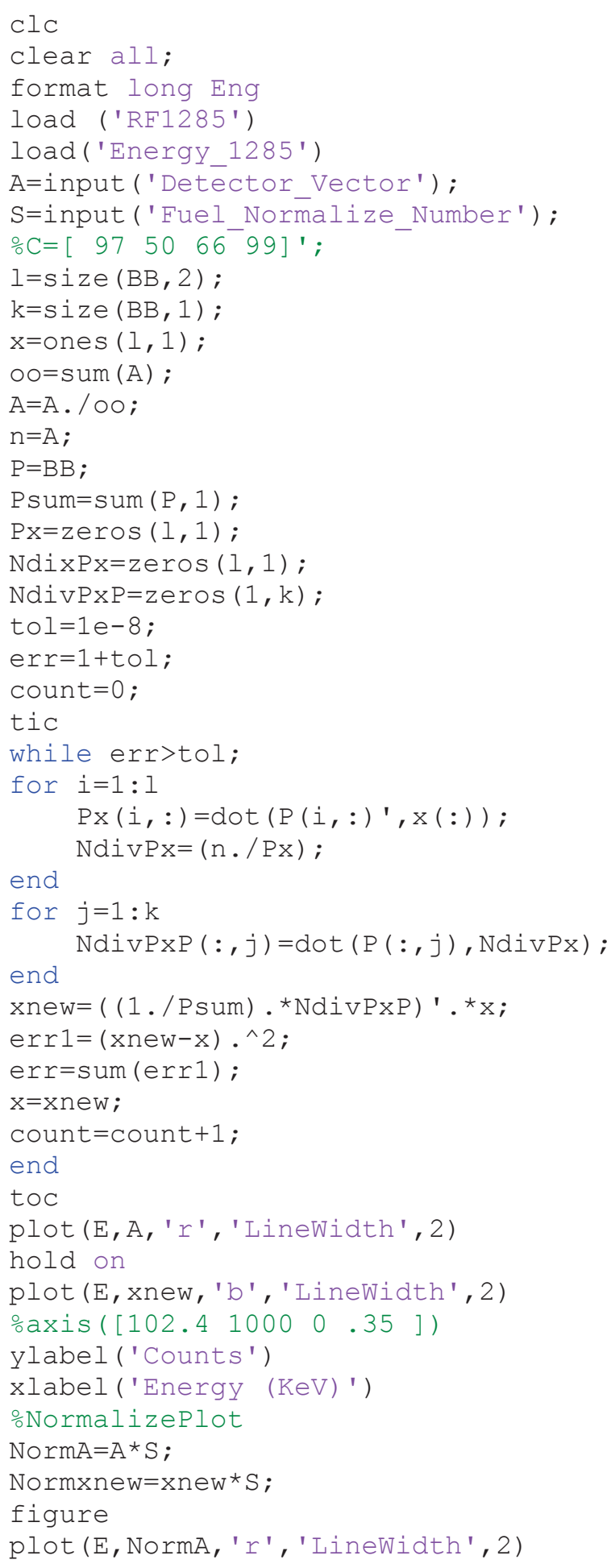


hold on

plot (E, Normxnew, 'b' , 'Linewidth', 2)

oaxis ([102.4 $10000 \quad .35$ ])

ylabel ( 'Counts')

xlabel ('Energy (KeV)')

uisave

( \{'xnew', 'err', 'A', 'tol', 'count', 'n', 'NormA', 'Normxnew'\}, 'Fuel_source ')

$\mathrm{Ex}=[$ NormA E Normxnew ];

xlswrite ('Fuel Deconvolution.xlsx',Ex, 'Xa379'); 
APPENDIX C

GAUSSIAN ANALYZER TOOL 


\section{Gaussian Analyzer}

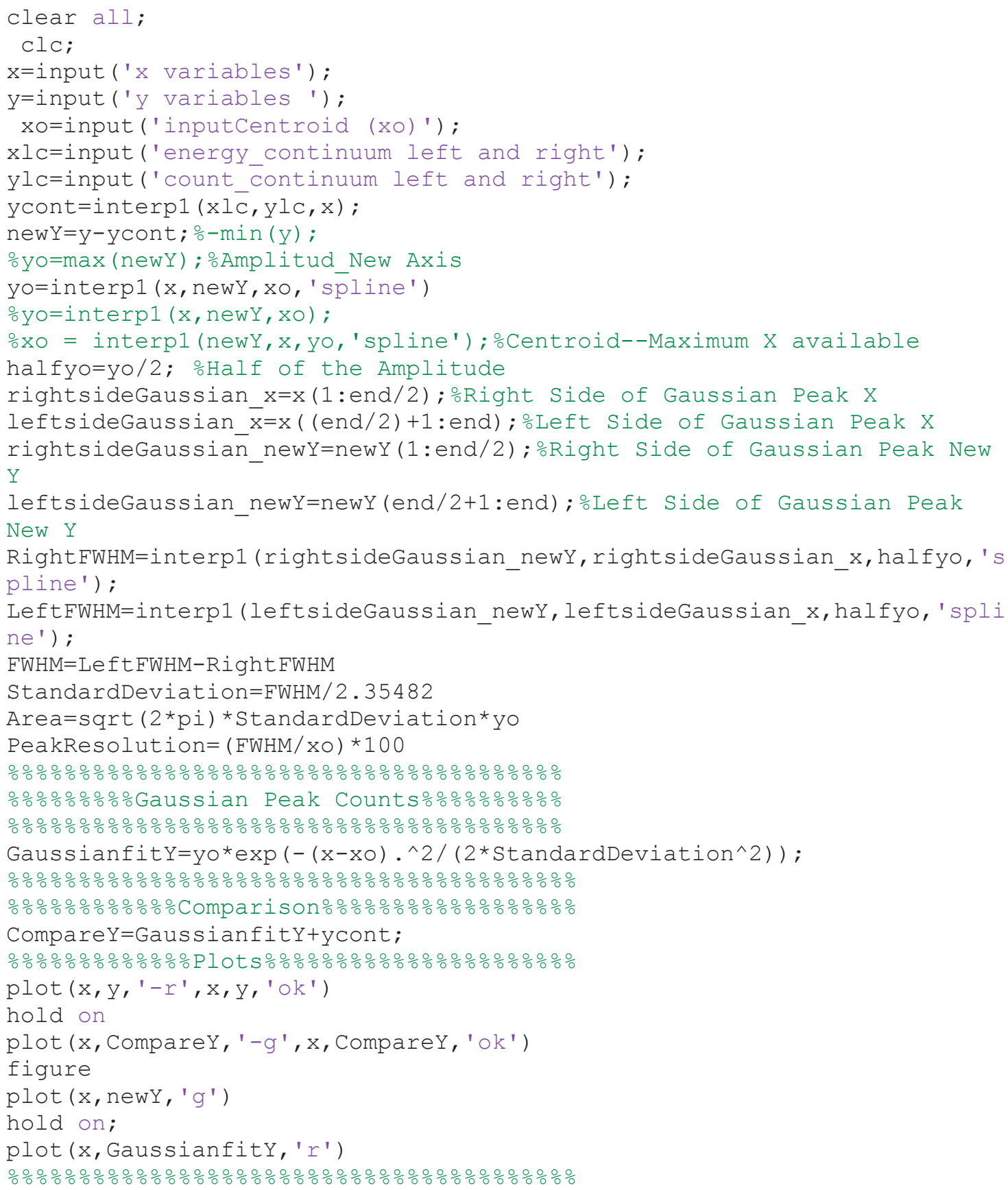


APPENDIX D

MCNPX FISSION WIRE RESPONSE

FUNCTION SAMPLE CODE 


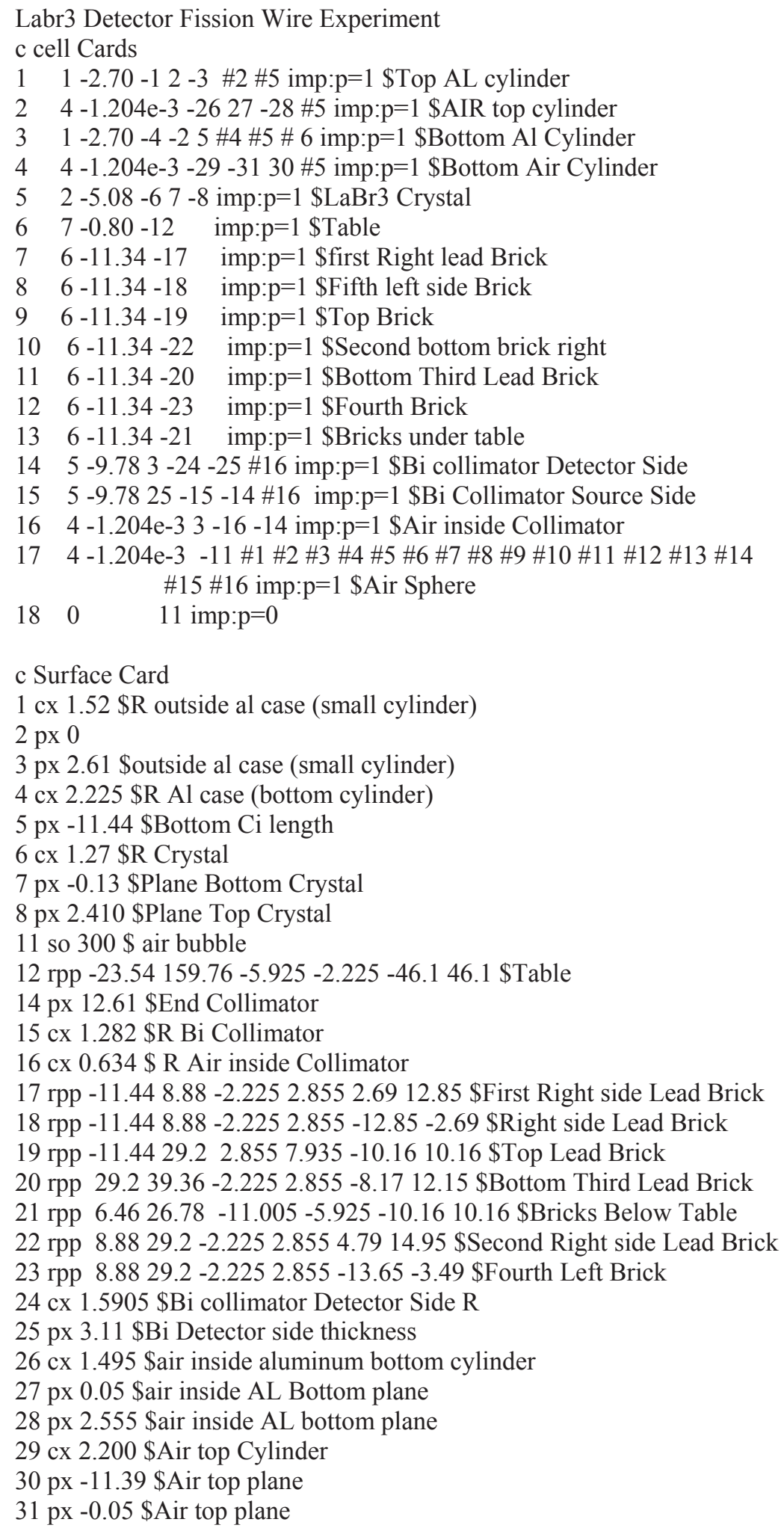




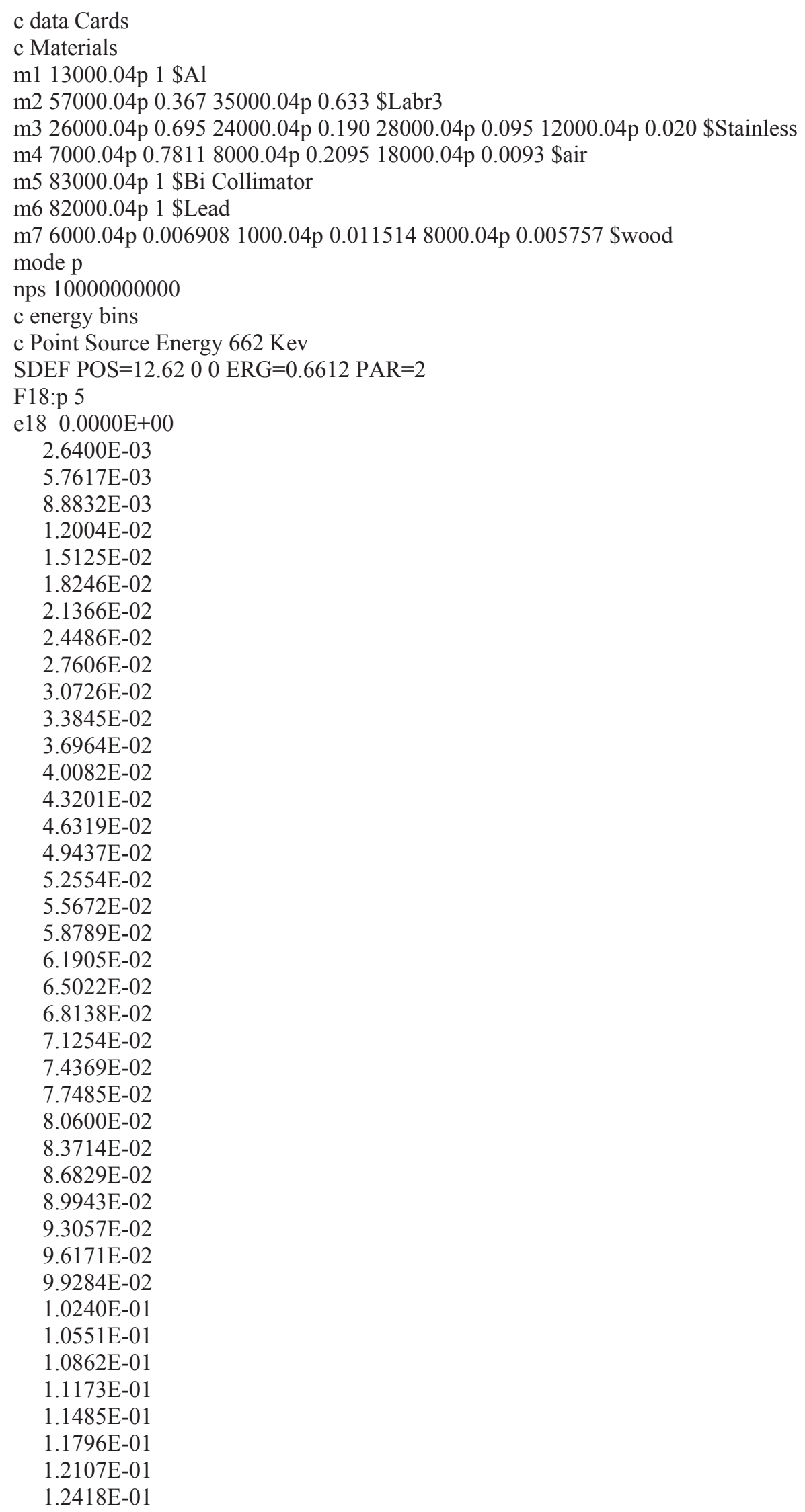




$$
\begin{aligned}
& 1.2729 \mathrm{E}-01 \\
& 1.3040 \mathrm{E}-01 \\
& 1.3351 \mathrm{E}-01 \\
& 1.3662 \mathrm{E}-01 \\
& 1.3973 \mathrm{E}-01 \\
& 1.4284 \mathrm{E}-01 \\
& 1.4595 \mathrm{E}-01 \\
& 1.4906 \mathrm{E}-01 \\
& 1.5217 \mathrm{E}-01 \\
& 1.5528 \mathrm{E}-01 \\
& 1.5839 \mathrm{E}-01 \\
& 1.6149 \mathrm{E}-01 \\
& 1.6460 \mathrm{E}-01 \\
& 1.6771 \mathrm{E}-01 \\
& 1.7081 \mathrm{E}-01 \\
& 1.7392 \mathrm{E}-01 \\
& 1.7703 \mathrm{E}-01 \\
& 1.8013 \mathrm{E}-01 \\
& 1.8324 \mathrm{E}-01 \\
& 1.8635 \mathrm{E}-01 \\
& 1.8945 \mathrm{E}-01 \\
& 1.9256 \mathrm{E}-01 \\
& 1.9566 \mathrm{E}-01 \\
& 1.9877 \mathrm{E}-01 \\
& 2.0187 \mathrm{E}-01 \\
& 2.0497 \mathrm{E}-01 \\
& 2.0808 \mathrm{E}-01 \\
& 2.1118 \mathrm{E}-01 \\
& 2.1428 \mathrm{E}-01 \\
& 2.1739 \mathrm{E}-01 \\
& 2.2049 \mathrm{E}-01 \\
& 2.2359 \mathrm{E}-01 \\
& 2.2669 \mathrm{E}-01 \\
& 2.2979 \mathrm{E}-01 \\
& 2.3290 \mathrm{E}-01 \\
& 2.3600 \mathrm{E}-01 \\
& 2.3910 \mathrm{E}-01 \\
& 2.4220 \mathrm{E}-01 \\
& 2.4530 \mathrm{E}-01 \\
& 2.4840 \mathrm{E}-01 \\
& 2.5150 \mathrm{E}-01 \\
& 2.5460 \mathrm{E}-01 \\
& 2.5770 \mathrm{E}-01 \\
& 2.6080 \mathrm{E}-01 \\
& 2.6389 \mathrm{E}-01 \\
& 2.6699 \mathrm{E}-01 \\
& 2.7009 \mathrm{E}-01 \\
& 2.7319 \mathrm{E}-01 \\
& 2.7629 \mathrm{E}-01 \\
& 2.7938 \mathrm{E}-01 \\
& 2.8248 \mathrm{E}-01 \\
& 2.8558 \mathrm{E}-01 \\
& 2.8867 \mathrm{E}-01 \\
& 2.9177 \mathrm{E}-01 \\
& 2.9487 \mathrm{E}-01 \\
& 2.9796 \mathrm{E}-01
\end{aligned}
$$




$$
\begin{aligned}
& 3.0106 \mathrm{E}-01 \\
& 3.0415 \mathrm{E}-01 \\
& 3.0725 \mathrm{E}-01 \\
& 3.1034 \mathrm{E}-01 \\
& 3.1343 \mathrm{E}-01 \\
& 3.1653 \mathrm{E}-01 \\
& 3.1962 \mathrm{E}-01 \\
& 3.2272 \mathrm{E}-01 \\
& 3.2581 \mathrm{E}-01 \\
& 3.2890 \mathrm{E}-01 \\
& 3.3199 \mathrm{E}-01 \\
& 3.3509 \mathrm{E}-01 \\
& 3.3818 \mathrm{E}-01 \\
& 3.4127 \mathrm{E}-01 \\
& 3.4436 \mathrm{E}-01 \\
& 3.4745 \mathrm{E}-01 \\
& 3.5054 \mathrm{E}-01 \\
& 3.5363 \mathrm{E}-01 \\
& 3.5672 \mathrm{E}-01 \\
& 3.5981 \mathrm{E}-01 \\
& 3.6290 \mathrm{E}-01 \\
& 3.6599 \mathrm{E}-01 \\
& 3.6908 \mathrm{E}-01 \\
& 3.7217 \mathrm{E}-01 \\
& 3.7526 \mathrm{E}-01 \\
& 3.7835 \mathrm{E}-01 \\
& 3.8144 \mathrm{E}-01 \\
& 3.8452 \mathrm{E}-01 \\
& 3.8761 \mathrm{E}-01 \\
& 3.9070 \mathrm{E}-01 \\
& 3.9379 \mathrm{E}-01 \\
& 3.9687 \mathrm{E}-01 \\
& 3.9996 \mathrm{E}-01 \\
& 4.0304 \mathrm{E}-01 \\
& 4.0613 \mathrm{E}-01 \\
& 4.0922 \mathrm{E}-01 \\
& 4.1230 \mathrm{E}-01 \\
& 4.1539 \mathrm{E}-01 \\
& 4.1847 \mathrm{E}-01 \\
& 4.2156 \mathrm{E}-01 \\
& 4.2464 \mathrm{E}-01 \\
& 4.2772 \mathrm{E}-01 \\
& 4.3081 \mathrm{E}-01 \\
& 4.3389 \mathrm{E}-01 \\
& 4.3697 \mathrm{E}-01 \\
& 4.4006 \mathrm{E}-01 \\
& 4.4314 \mathrm{E}-01 \\
& 4.4622 \mathrm{E}-01 \\
& 4.4930 \mathrm{E}-01 \\
& 4.5238 \mathrm{E}-01 \\
& 4.5547 \mathrm{E}-01 \\
& 4.5855 \mathrm{E}-01 \\
& 4.6163 \mathrm{E}-01 \\
& 4.6471 \mathrm{E}-01 \\
& 4.6779 \mathrm{E}-01 \\
& 4.7087 \mathrm{E}-01
\end{aligned}
$$




$$
\begin{aligned}
& 4.7395 \mathrm{E}-01 \\
& 4.7703 \mathrm{E}-01 \\
& 4.8011 \mathrm{E}-01 \\
& 4.8319 \mathrm{E}-01 \\
& 4.8626 \mathrm{E}-01 \\
& 4.8934 \mathrm{E}-01 \\
& 4.9242 \mathrm{E}-01 \\
& 4.9550 \mathrm{E}-01 \\
& 4.9858 \mathrm{E}-01 \\
& 5.0165 \mathrm{E}-01 \\
& 5.0473 \mathrm{E}-01 \\
& 5.0781 \mathrm{E}-01 \\
& 5.1088 \mathrm{E}-01 \\
& 5.1396 \mathrm{E}-01 \\
& 5.1703 \mathrm{E}-01 \\
& 5.2011 \mathrm{E}-01 \\
& 5.2319 \mathrm{E}-01 \\
& 5.2626 \mathrm{E}-01 \\
& 5.2933 \mathrm{E}-01 \\
& 5.3241 \mathrm{E}-01 \\
& 5.3548 \mathrm{E}-01 \\
& 5.3856 \mathrm{E}-01 \\
& 5.4163 \mathrm{E}-01 \\
& 5.4470 \mathrm{E}-01 \\
& 5.4778 \mathrm{E}-01 \\
& 5.5085 \mathrm{E}-01 \\
& 5.5392 \mathrm{E}-01 \\
& 5.5699 \mathrm{E}-01 \\
& 5.6007 \mathrm{E}-01 \\
& 5.6314 \mathrm{E}-01 \\
& 5.6621 \mathrm{E}-01 \\
& 5.6928 \mathrm{E}-01 \\
& 5.7235 \mathrm{E}-01 \\
& 5.7542 \mathrm{E}-01 \\
& 5.7849 \mathrm{E}-01 \\
& 5.8156 \mathrm{E}-01 \\
& 5.8463 \mathrm{E}-01 \\
& 5.8770 \mathrm{E}-01 \\
& 5.9077 \mathrm{E}-01 \\
& 5.9384 \mathrm{E}-01 \\
& 5.9691 \mathrm{E}-01 \\
& 5.9998 \mathrm{E}-01 \\
& 6.0304 \mathrm{E}-01 \\
& 6.0611 \mathrm{E}-01 \\
& 6.0918 \mathrm{E}-01 \\
& 6.1225 \mathrm{E}-01 \\
& 6.1531 \mathrm{E}-01 \\
& 6.1838 \mathrm{E}-01 \\
& 6.2145 \mathrm{E}-01 \\
& 6.2451 \mathrm{E}-01 \\
& 6.2758 \mathrm{E}-01 \\
& 6.3064 \mathrm{E}-01 \\
& 6.3371 \mathrm{E}-01 \\
& 6.3677 \mathrm{E}-01 \\
& 6.3984 \mathrm{E}-01 \\
& 6.4290 \mathrm{E}-01
\end{aligned}
$$




$$
\begin{aligned}
& 6.4597 \mathrm{E}-01 \\
& 6.4903 \mathrm{E}-01 \\
& 6.5209 \mathrm{E}-01 \\
& 6.5516 \mathrm{E}-01 \\
& 6.5822 \mathrm{E}-01 \\
& 6.6128 \mathrm{E}-01 \\
& 6.6434 \mathrm{E}-01 \\
& 6.6741 \mathrm{E}-01 \\
& 6.7047 \mathrm{E}-01 \\
& 6.7353 \mathrm{E}-01 \\
& 6.7659 \mathrm{E}-01 \\
& 6.7965 \mathrm{E}-01 \\
& 6.8271 \mathrm{E}-01 \\
& 6.8577 \mathrm{E}-01 \\
& 6.8883 \mathrm{E}-01 \\
& 6.9189 \mathrm{E}-01 \\
& 6.9495 \mathrm{E}-01 \\
& 6.9801 \mathrm{E}-01 \\
& 7.0107 \mathrm{E}-01 \\
& 7.0413 \mathrm{E}-01 \\
& 7.0719 \mathrm{E}-01 \\
& 7.1025 \mathrm{E}-01 \\
& 7.1331 \mathrm{E}-01 \\
& 7.1636 \mathrm{E}-01 \\
& 7.1942 \mathrm{E}-01 \\
& 7.2248 \mathrm{E}-01 \\
& 7.2554 \mathrm{E}-01 \\
& 7.2859 \mathrm{E}-01 \\
& 7.3165 \mathrm{E}-01 \\
& 7.3470 \mathrm{E}-01 \\
& 7.3776 \mathrm{E}-01 \\
& 7.4082 \mathrm{E}-01 \\
& 7.4387 \mathrm{E}-01 \\
& 7.4693 \mathrm{E}-01 \\
& 7.4998 \mathrm{E}-01 \\
& 7.5303 \mathrm{E}-01 \\
& 7.5609 \mathrm{E}-01 \\
& 7.5914 \mathrm{E}-01 \\
& 7.6220 \mathrm{E}-01 \\
& 7.6525 \mathrm{E}-01 \\
& 7.6830 \mathrm{E}-01 \\
& 7.7135 \mathrm{E}-01 \\
& 7.7441 \mathrm{E}-01 \\
& 7.7746 \mathrm{E}-01 \\
& 7.8051 \mathrm{E}-01 \\
& 7.8356 \mathrm{E}-01 \\
& 7.8661 \mathrm{E}-01 \\
& 7.8966 \mathrm{E}-01 \\
& 7.9272 \mathrm{E}-01 \\
& 7.9577 \mathrm{E}-01 \\
& 7.9882 \mathrm{E}-01 \\
& 8.0187 \mathrm{E}-01 \\
& 8.0492 \mathrm{E}-01 \\
& 8.0797 \mathrm{E}-01 \\
& 8.1101 \mathrm{E}-01 \\
& 8.1406 \mathrm{E}-01 \\
& \\
&
\end{aligned}
$$




$$
\begin{aligned}
& 8.1711 \mathrm{E}-01 \\
& 8.2016 \mathrm{E}-01 \\
& 8.2321 \mathrm{E}-01 \\
& 8.2626 \mathrm{E}-01 \\
& 8.2930 \mathrm{E}-01 \\
& 8.3235 \mathrm{E}-01 \\
& 8.3540 \mathrm{E}-01 \\
& 8.3844 \mathrm{E}-01 \\
& 8.4149 \mathrm{E}-01 \\
& 8.4454 \mathrm{E}-01 \\
& 8.4758 \mathrm{E}-01 \\
& 8.5063 \mathrm{E}-01 \\
& 8.5367 \mathrm{E}-01 \\
& 8.5672 \mathrm{E}-01 \\
& 8.5976 \mathrm{E}-01 \\
& 8.6281 \mathrm{E}-01 \\
& 8.6585 \mathrm{E}-01 \\
& 8.6889 \mathrm{E}-01 \\
& 8.7194 \mathrm{E}-01 \\
& 8.7498 \mathrm{E}-01 \\
& 8.7802 \mathrm{E}-01 \\
& 8.8107 \mathrm{E}-01 \\
& 8.8411 \mathrm{E}-01 \\
& 8.8715 \mathrm{E}-01 \\
& 8.9019 \mathrm{E}-01 \\
& 8.9323 \mathrm{E}-01 \\
& 8.9628 \mathrm{E}-01 \\
& 8.9932 \mathrm{E}-01 \\
& 9.0236 \mathrm{E}-01 \\
& 9.0540 \mathrm{E}-01 \\
& 9.0844 \mathrm{E}-01 \\
& 9.1148 \mathrm{E}-01 \\
& 9.1452 \mathrm{E}-01 \\
& 9.1756 \mathrm{E}-01 \\
& 9.2060 \mathrm{E}-01 \\
& 9.2363 \mathrm{E}-01 \\
& 9.2667 \mathrm{E}-01 \\
& 9.2971 \mathrm{E}-01 \\
& 9.3275 \mathrm{E}-01 \\
& 9.3579 \mathrm{E}-01 \\
& 9.3882 \mathrm{E}-01 \\
& 9.4186 \mathrm{E}-01 \\
& 9.4490 \mathrm{E}-01 \\
& 9.4793 \mathrm{E}-01 \\
& 9.5097 \mathrm{E}-01 \\
& 9.5401 \mathrm{E}-01 \\
& 9.5704 \mathrm{E}-01 \\
& 9.6008 \mathrm{E}-01 \\
& 9.6311 \mathrm{E}-01 \\
& 9.6615 \mathrm{E}-01 \\
& 9.6918 \mathrm{E}-01 \\
& 9.7222 \mathrm{E}-01 \\
& 9.7525 \mathrm{E}-01 \\
& 9.7828 \mathrm{E}-01 \\
& 9.8132 \mathrm{E}-01 \\
& 9.8435 \mathrm{E}-01
\end{aligned}
$$


$9.8738 \mathrm{E}-01$
$9.9042 \mathrm{E}-01$
$9.9345 \mathrm{E}-01$
$9.9648 \mathrm{E}-01$
$9.9951 \mathrm{E}-01$
$1.0025 \mathrm{E}+00$
$1.0056 \mathrm{E}+00$
$1.0086 \mathrm{E}+00$
$1.0116 \mathrm{E}+00$
$1.0147 \mathrm{E}+00$
$1.0177 \mathrm{E}+00$
$1.0207 \mathrm{E}+00$
$1.0238 \mathrm{E}+00$
$1.0268 \mathrm{E}+00$
$1.0298 \mathrm{E}+00$
$1.0328 \mathrm{E}+00$
$1.0359 \mathrm{E}+00$
$1.0389 \mathrm{E}+00$
$1.0419 \mathrm{E}+00$
$1.0450 \mathrm{E}+00$
$1.0480 \mathrm{E}+00$
$1.0510 \mathrm{E}+00$
$1.0540 \mathrm{E}+00$
$1.0571 \mathrm{E}+00$
$1.0601 \mathrm{E}+00$
$1.0631 \mathrm{E}+00$
$1.0661 \mathrm{E}+00$
$1.0692 \mathrm{E}+00$
$1.0722 \mathrm{E}+00$
$1.0752 \mathrm{E}+00$
$1.0782 \mathrm{E}+00$
$1.0813 \mathrm{E}+00$
$1.0843 \mathrm{E}+00$
$1.0873 \mathrm{E}+00$
$1.0903 \mathrm{E}+00$
$1.0934 \mathrm{E}+00$
$1.0964 \mathrm{E}+00$
$1.0994 \mathrm{E}+00$
$1.1024 \mathrm{E}+00$
$1.1055 \mathrm{E}+00$
$1.1085 \mathrm{E}+00$
$1.1115 \mathrm{E}+00$
$1.1145 \mathrm{E}+00$
$1.1175 \mathrm{E}+00$
$1.1206 \mathrm{E}+00$
$1.1236 \mathrm{E}+00$
$1.1266 \mathrm{E}+00$
$1.1296 \mathrm{E}+00$
$1.1326 \mathrm{E}+00$
$1.1357 \mathrm{E}+00$
$1.1387 \mathrm{E}+00$
$1.1417 \mathrm{E}+00$
$1.1447 \mathrm{E}+00$
$1.1477 \mathrm{E}+00$
$1.1507 \mathrm{E}+00$
$1.1538 \mathrm{E}+00$ 
$1.1568 \mathrm{E}+00$
$1.1598 \mathrm{E}+00$
$1.1628 \mathrm{E}+00$
$1.1658 \mathrm{E}+00$
$1.1688 \mathrm{E}+00$
$1.1719 \mathrm{E}+00$
$1.1749 \mathrm{E}+00$
$1.1779 \mathrm{E}+00$
$1.1809 \mathrm{E}+00$
$1.1839 \mathrm{E}+00$
$1.1869 \mathrm{E}+00$
$1.1900 \mathrm{E}+00$
$1.1930 \mathrm{E}+00$
$1.1960 \mathrm{E}+00$
$1.1990 \mathrm{E}+00$
$1.2020 \mathrm{E}+00$
$1.2050 \mathrm{E}+00$
$1.2080 \mathrm{E}+00$
$1.2110 \mathrm{E}+00$
$1.2141 \mathrm{E}+00$
$1.2171 \mathrm{E}+00$
$1.2201 \mathrm{E}+00$
$1.2231 \mathrm{E}+00$
$1.2261 \mathrm{E}+00$
$1.2291 \mathrm{E}+00$
$1.2321 \mathrm{E}+00$
$1.2351 \mathrm{E}+00$
$1.2381 \mathrm{E}+00$
$1.2412 \mathrm{E}+00$
$1.2442 \mathrm{E}+00$
$1.2472 \mathrm{E}+00$
$1.2502 \mathrm{E}+00$
$1.2532 \mathrm{E}+00$
$1.2562 \mathrm{E}+00$
$1.2592 \mathrm{E}+00$
$1.2622 \mathrm{E}+00$
$1.2652 \mathrm{E}+00$
$1.2682 \mathrm{E}+00$
$1.2712 \mathrm{E}+00$
$1.2742 \mathrm{E}+00$
$1.2772 \mathrm{E}+00$
$1.2803 \mathrm{E}+00$
$1.2833 \mathrm{E}+00$
$1.2863 \mathrm{E}+00$
$1.2893 \mathrm{E}+00$
$1.2923 \mathrm{E}+00$
$1.2953 \mathrm{E}+00$
$1.2983 \mathrm{E}+00$
$1.3013 \mathrm{E}+00$
$1.3043 \mathrm{E}+00$
$1.3073 \mathrm{E}+00$
$1.3103 \mathrm{E}+00$
$1.3133 \mathrm{E}+00$
$1.3163 \mathrm{E}+00$
$1.3193 \mathrm{E}+00$
$1.3223 \mathrm{E}+00$ 


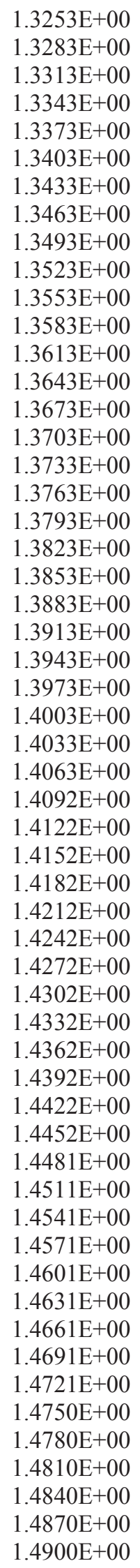




$$
\begin{aligned}
& 1.4930 \mathrm{E}+00 \\
& 1.4959 \mathrm{E}+00 \\
& 1.4989 \mathrm{E}+00 \\
& 1.5019 \mathrm{E}+00 \\
& 1.5049 \mathrm{E}+00 \\
& 1.5079 \mathrm{E}+00 \\
& 1.5109 \mathrm{E}+00 \\
& 1.5139 \mathrm{E}+00 \\
& 1.5168 \mathrm{E}+00 \\
& 1.5198 \mathrm{E}+00 \\
& 1.5228 \mathrm{E}+00 \\
& 1.5258 \mathrm{E}+00 \\
& 1.5288 \mathrm{E}+00 \\
& 1.5318 \mathrm{E}+00 \\
& 1.5347 \mathrm{E}+00 \\
& 1.5377 \mathrm{E}+00 \\
& 1.5407 \mathrm{E}+00 \\
& 1.5437 \mathrm{E}+00 \\
& 1.5467 \mathrm{E}+00 \\
& 1.5496 \mathrm{E}+00 \\
& 1.5526 \mathrm{E}+00 \\
& 1.5556 \mathrm{E}+00 \\
& 1.5586 \mathrm{E}+00 \\
& 1.5616 \mathrm{E}+00 \\
& 1.5645 \mathrm{E}+00 \\
& 1.5675 \mathrm{E}+00 \\
& 1.5705 \mathrm{E}+00 \\
& 1.5735 \mathrm{E}+00 \\
& 1.5765 \mathrm{E}+00 \\
& 1.5794 \mathrm{E}+00 \\
& 1.5824 \mathrm{E}+00 \\
& 1.5854 \mathrm{E}+00 \\
& 1.5884 \mathrm{E}+00 \\
& 1.5913 \mathrm{E}+00 \\
& 1.5943 \mathrm{E}+00 \\
& 1.5973 \mathrm{E}+00 \\
& 1.6003 \mathrm{E}+00 \\
& 1.6033 \mathrm{E}+00 \\
& 1.6062 \mathrm{E}+00 \\
& 1.6092 \mathrm{E}+00 \\
& 1.6122 \mathrm{E}+00 \\
& 1.6152 \mathrm{E}+00 \\
& 1.6181 \mathrm{E}+00 \\
& 1.6211 \mathrm{E}+00 \\
& 1.6241 \mathrm{E}+00 \\
& 1.6271 \mathrm{E}+00 \\
& 1.6300 \mathrm{E}+00 \\
& 1.6330 \mathrm{E}+00 \\
& 1.6360 \mathrm{E}+00 \\
& 1.6389 \mathrm{E}+00 \\
& 1.6419 \mathrm{E}+00 \\
& 1.6449 \mathrm{E}+00 \\
& 1.6479 \mathrm{E}+00 \\
& 1.6508 \mathrm{E}+00 \\
& 1.6538 \mathrm{E}+00 \\
& 1.6568 \mathrm{E}+00
\end{aligned}
$$




$$
\begin{aligned}
& 1.6597 \mathrm{E}+00 \\
& 1.6627 \mathrm{E}+00 \\
& 1.6657 \mathrm{E}+00 \\
& 1.6687 \mathrm{E}+00 \\
& 1.6716 \mathrm{E}+00 \\
& 1.6746 \mathrm{E}+00 \\
& 1.6776 \mathrm{E}+00 \\
& 1.6805 \mathrm{E}+00 \\
& 1.6835 \mathrm{E}+00 \\
& 1.6865 \mathrm{E}+00 \\
& 1.6894 \mathrm{E}+00 \\
& 1.6924 \mathrm{E}+00 \\
& 1.6954 \mathrm{E}+00 \\
& 1.6983 \mathrm{E}+00 \\
& 1.7013 \mathrm{E}+00 \\
& 1.7043 \mathrm{E}+00 \\
& 1.7072 \mathrm{E}+00 \\
& 1.7102 \mathrm{E}+00 \\
& 1.7132 \mathrm{E}+00 \\
& 1.7161 \mathrm{E}+00 \\
& 1.7191 \mathrm{E}+00 \\
& 1.7221 \mathrm{E}+00 \\
& 1.7250 \mathrm{E}+00 \\
& 1.7280 \mathrm{E}+00 \\
& 1.7310 \mathrm{E}+00 \\
& 1.7339 \mathrm{E}+00 \\
& 1.7369 \mathrm{E}+00 \\
& 1.7398 \mathrm{E}+00 \\
& 1.7428 \mathrm{E}+00 \\
& 1.7458 \mathrm{E}+00 \\
& 1.7487 \mathrm{E}+00 \\
& 1.7517 \mathrm{E}+00 \\
& 1.7547 \mathrm{E}+00 \\
& 1.7576 \mathrm{E}+00 \\
& 1.7606 \mathrm{E}+00 \\
& 1.7635 \mathrm{E}+00 \\
& 1.7665 \mathrm{E}+00 \\
& 1.7695 \mathrm{E}+00 \\
& 1.7724 \mathrm{E}+00 \\
& 1.7754 \mathrm{E}+00 \\
& 1.7783 \mathrm{E}+00 \\
& 1.7813 \mathrm{E}+00 \\
& 1.7843 \mathrm{E}+00 \\
& 1.7872 \mathrm{E}+00 \\
& 1.7902 \mathrm{E}+00 \\
& 1.7931 \mathrm{E}+00 \\
& 1.7961 \mathrm{E}+00 \\
& 1.7990 \mathrm{E}+00 \\
& 1.8020 \mathrm{E}+00 \\
& 1.8050 \mathrm{E}+00 \\
& 1.8079 \mathrm{E}+00 \\
& 1.8109 \mathrm{E}+00 \\
& 1.8138 \mathrm{E}+00 \\
& 1.8168 \mathrm{E}+00 \\
& 1.8197 \mathrm{E}+00 \\
& 1.8227 \mathrm{E}+00
\end{aligned}
$$




$$
\begin{aligned}
& 1.8256 \mathrm{E}+00 \\
& 1.8286 \mathrm{E}+00 \\
& 1.8316 \mathrm{E}+00 \\
& 1.8345 \mathrm{E}+00 \\
& 1.8375 \mathrm{E}+00 \\
& 1.8404 \mathrm{E}+00 \\
& 1.8434 \mathrm{E}+00 \\
& 1.8463 \mathrm{E}+00 \\
& 1.8493 \mathrm{E}+00 \\
& 1.8522 \mathrm{E}+00 \\
& 1.8552 \mathrm{E}+00 \\
& 1.8581 \mathrm{E}+00 \\
& 1.8611 \mathrm{E}+00 \\
& 1.8640 \mathrm{E}+00 \\
& 1.8670 \mathrm{E}+00 \\
& 1.8699 \mathrm{E}+00 \\
& 1.8729 \mathrm{E}+00 \\
& 1.8758 \mathrm{E}+00 \\
& 1.8788 \mathrm{E}+00 \\
& 1.8817 \mathrm{E}+00 \\
& 1.8847 \mathrm{E}+00 \\
& 1.8876 \mathrm{E}+00 \\
& 1.8906 \mathrm{E}+00 \\
& 1.8935 \mathrm{E}+00 \\
& 1.8965 \mathrm{E}+00 \\
& 1.8994 \mathrm{E}+00 \\
& 1.9024 \mathrm{E}+00 \\
& 1.9053 \mathrm{E}+00 \\
& 1.9083 \mathrm{E}+00 \\
& 1.9112 \mathrm{E}+00 \\
& 1.9142 \mathrm{E}+00 \\
& 1.9171 \mathrm{E}+00 \\
& 1.9201 \mathrm{E}+00 \\
& 1.9230 \mathrm{E}+00 \\
& 1.9260 \mathrm{E}+00 \\
& 1.9289 \mathrm{E}+00 \\
& 1.9318 \mathrm{E}+00 \\
& 1.9348 \mathrm{E}+00 \\
& 1.9377 \mathrm{E}+00 \\
& 1.9407 \mathrm{E}+00 \\
& 1.9436 \mathrm{E}+00 \\
& 1.9466 \mathrm{E}+00 \\
& 1.9495 \mathrm{E}+00 \\
& 1.9524 \mathrm{E}+00 \\
& 1.9554 \mathrm{E}+00 \\
& 1.9583 \mathrm{E}+00 \\
& 1.9613 \mathrm{E}+00 \\
& 1.9642 \mathrm{E}+00 \\
& 1.9672 \mathrm{E}+00 \\
& 1.9701 \mathrm{E}+00 \\
& 1.9730 \mathrm{E}+00 \\
& 1.9760 \mathrm{E}+00 \\
& 1.9789 \mathrm{E}+00 \\
& 1.9819 \mathrm{E}+00 \\
& 1.9848 \mathrm{E}+00 \\
& 1.9877 \mathrm{E}+00
\end{aligned}
$$




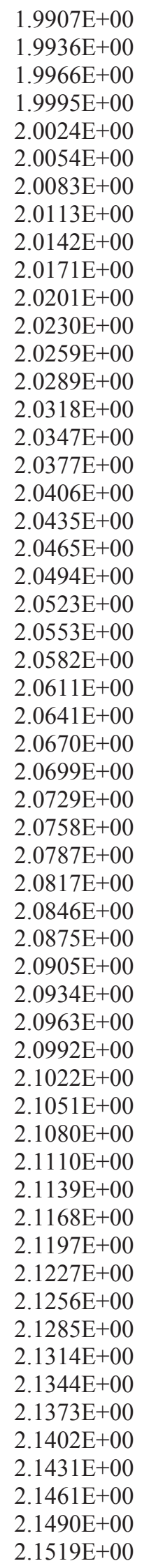


$2.1548 \mathrm{E}+00$

$2.1578 \mathrm{E}+00$

$2.1607 \mathrm{E}+00$

$2.1636 \mathrm{E}+00$

$2.1665 \mathrm{E}+00$

$2.1695 \mathrm{E}+00$

$2.1724 \mathrm{E}+00$

$2.1753 \mathrm{E}+00$

$2.1782 \mathrm{E}+00$

$2.1811 \mathrm{E}+00$

$2.1841 \mathrm{E}+00$

$2.1870 \mathrm{E}+00$

$2.1899 \mathrm{E}+00$

$2.1928 \mathrm{E}+00$

$2.1957 \mathrm{E}+00$

$2.1987 \mathrm{E}+00$

$2.2016 \mathrm{E}+00$

$2.2045 \mathrm{E}+00$

$2.2074 \mathrm{E}+00$

$2.2103 \mathrm{E}+00$

$2.2133 \mathrm{E}+00$

$2.2162 \mathrm{E}+00$

$2.2191 \mathrm{E}+00$

$2.2220 \mathrm{E}+00$

$2.2249 \mathrm{E}+00$

$2.2278 \mathrm{E}+00$

$2.2308 \mathrm{E}+00$

$2.2337 \mathrm{E}+00$

$2.2366 \mathrm{E}+00$

$2.2395 \mathrm{E}+00$

$2.2424 \mathrm{E}+00$

$2.2453 \mathrm{E}+00$

$2.2483 \mathrm{E}+00$

$2.2512 \mathrm{E}+00$

$2.2541 \mathrm{E}+00$

$2.2570 \mathrm{E}+00$

$2.2599 \mathrm{E}+00$

$2.2628 \mathrm{E}+00$

$2.2657 \mathrm{E}+00$

$2.2687 \mathrm{E}+00$

$2.2716 \mathrm{E}+00$

$2.2745 \mathrm{E}+00$

$2.2774 \mathrm{E}+00$

$2.2803 \mathrm{E}+00$

$2.2832 \mathrm{E}+00$

$2.2861 \mathrm{E}+00$

$2.2890 \mathrm{E}+00$

$2.2919 \mathrm{E}+00$

$2.2949 \mathrm{E}+00$

$2.2978 \mathrm{E}+00$

$2.3007 \mathrm{E}+00$

$2.3036 \mathrm{E}+00$

$2.3065 \mathrm{E}+00$

$2.3094 \mathrm{E}+00$

$2.3123 \mathrm{E}+00$

$2.3152 \mathrm{E}+00$ 
$2.3181 \mathrm{E}+00$
$2.3210 \mathrm{E}+00$
$2.3239 \mathrm{E}+00$
$2.3269 \mathrm{E}+00$
$2.3298 \mathrm{E}+00$
$2.3327 \mathrm{E}+00$
$2.3356 \mathrm{E}+00$
$2.3385 \mathrm{E}+00$
$2.3414 \mathrm{E}+00$
$2.3443 \mathrm{E}+00$
$2.3472 \mathrm{E}+00$
$2.3501 \mathrm{E}+00$
$2.3530 \mathrm{E}+00$
$2.3559 \mathrm{E}+00$
$2.3588 \mathrm{E}+00$
$2.3617 \mathrm{E}+00$
$2.3646 \mathrm{E}+00$
$2.3675 \mathrm{E}+00$
$2.3704 \mathrm{E}+00$
$2.3733 \mathrm{E}+00$
$2.3762 \mathrm{E}+00$
$2.3791 \mathrm{E}+00$
$2.3820 \mathrm{E}+00$
$2.3849 \mathrm{E}+00$
$2.3878 \mathrm{E}+00$
$2.3907 \mathrm{E}+00$
$2.3936 \mathrm{E}+00$
$2.3965 \mathrm{E}+00$
$2.3994 \mathrm{E}+00$
$2.4023 \mathrm{E}+00$
$2.4052 \mathrm{E}+00$
$2.4081 \mathrm{E}+00$
$2.4110 \mathrm{E}+00$
$2.4139 \mathrm{E}+00$
$2.4168 \mathrm{E}+00$
$2.4197 \mathrm{E}+00$
$2.4226 \mathrm{E}+00$
$2.4255 \mathrm{E}+00$
$2.4284 \mathrm{E}+00$
$2.4313 \mathrm{E}+00$
$2.4342 \mathrm{E}+00$
$2.4371 \mathrm{E}+00$
$2.4400 \mathrm{E}+00$
$2.4429 \mathrm{E}+00$
$2.4458 \mathrm{E}+00$
$2.4487 \mathrm{E}+00$
$2.4516 \mathrm{E}+00$
$2.4545 \mathrm{E}+00$
$2.4574 \mathrm{E}+00$
$2.4603 \mathrm{E}+00$
$2.4632 \mathrm{E}+00$
$2.4661 \mathrm{E}+00$
$2.4690 \mathrm{E}+00$
$2.4719 \mathrm{E}+00$
$2.4748 \mathrm{E}+00$
$2.4777 \mathrm{E}+00$ 
$2.4805 \mathrm{E}+00$
$2.4834 \mathrm{E}+00$
$2.4863 \mathrm{E}+00$
$2.4892 \mathrm{E}+00$
$2.4921 \mathrm{E}+00$
$2.4950 \mathrm{E}+00$
$2.4979 \mathrm{E}+00$
$2.5008 \mathrm{E}+00$
$2.5037 \mathrm{E}+00$
$2.5066 \mathrm{E}+00$
$2.5095 \mathrm{E}+00$
$2.5123 \mathrm{E}+00$
$2.5152 \mathrm{E}+00$
$2.5181 \mathrm{E}+00$
$2.5210 \mathrm{E}+00$
$2.5239 \mathrm{E}+00$
$2.5268 \mathrm{E}+00$
$2.5297 \mathrm{E}+00$
$2.5326 \mathrm{E}+00$
$2.5355 \mathrm{E}+00$
$2.5383 \mathrm{E}+00$
$2.5412 \mathrm{E}+00$
$2.5441 \mathrm{E}+00$
$2.5470 \mathrm{E}+00$
$2.5499 \mathrm{E}+00$
$2.5528 \mathrm{E}+00$
$2.5557 \mathrm{E}+00$
$2.5585 \mathrm{E}+00$
$2.5614 \mathrm{E}+00$
$2.5643 \mathrm{E}+00$
$2.5672 \mathrm{E}+00$
$2.5701 \mathrm{E}+00$
$2.5730 \mathrm{E}+00$
$2.5758 \mathrm{E}+00$
$2.5787 \mathrm{E}+00$
$2.5816 \mathrm{E}+00$
$2.5845 \mathrm{E}+00$
$2.5874 \mathrm{E}+00$
$2.5903 \mathrm{E}+00$
$2.5931 \mathrm{E}+00$
$2.5960 \mathrm{E}+00$
$2.5989 \mathrm{E}+00$
$2.6018 \mathrm{E}+00$
$2.6047 \mathrm{E}+00$
$2.6075 \mathrm{E}+00$
$2.6104 \mathrm{E}+00$
$2.6133 \mathrm{E}+00$
$2.6162 \mathrm{E}+00$
$2.6191 \mathrm{E}+00$
$2.6219 \mathrm{E}+00$
$2.6248 \mathrm{E}+00$
$2.6277 \mathrm{E}+00$
$2.6306 \mathrm{E}+00$
$2.6335 \mathrm{E}+00$
$2.6363 \mathrm{E}+00$
$2.6392 \mathrm{E}+00$ 
$2.6421 \mathrm{E}+00$
$2.6450 \mathrm{E}+00$
$2.6478 \mathrm{E}+00$
$2.6507 \mathrm{E}+00$
$2.6536 \mathrm{E}+00$
$2.6565 \mathrm{E}+00$
$2.6593 \mathrm{E}+00$
$2.6622 \mathrm{E}+00$
$2.6651 \mathrm{E}+00$
$2.6680 \mathrm{E}+00$
$2.6708 \mathrm{E}+00$
$2.6737 \mathrm{E}+00$
$2.6766 \mathrm{E}+00$
$2.6795 \mathrm{E}+00$
$2.6823 \mathrm{E}+00$
$2.6852 \mathrm{E}+00$
$2.6881 \mathrm{E}+00$
$2.6910 \mathrm{E}+00$
$2.6938 \mathrm{E}+00$
$2.6967 \mathrm{E}+00$
$2.6996 \mathrm{E}+00$
$2.7024 \mathrm{E}+00$
$2.7053 \mathrm{E}+00$
$2.7082 \mathrm{E}+00$
$2.7111 \mathrm{E}+00$
$2.7139 \mathrm{E}+00$
$2.7168 \mathrm{E}+00$
$2.7197 \mathrm{E}+00$
$2.7225 \mathrm{E}+00$
$2.7254 \mathrm{E}+00$
$2.7283 \mathrm{E}+00$
$2.7311 \mathrm{E}+00$
$2.7340 \mathrm{E}+00$
$2.7369 \mathrm{E}+00$
$2.7397 \mathrm{E}+00$
$2.7426 \mathrm{E}+00$
$2.7455 \mathrm{E}+00$
$2.7483 \mathrm{E}+00$
$2.7512 \mathrm{E}+00$
$2.7541 \mathrm{E}+00$
$2.7569 \mathrm{E}+00$
$2.7598 \mathrm{E}+00$
$2.7627 \mathrm{E}+00$
$2.7655 \mathrm{E}+00$
$2.7684 \mathrm{E}+00$
$2.7713 \mathrm{E}+00$
$2.7741 \mathrm{E}+00$
$2.7770 \mathrm{E}+00$
$2.7799 \mathrm{E}+00$
$2.7827 \mathrm{E}+00$
$2.7856 \mathrm{E}+00$
$2.7884 \mathrm{E}+00$
$2.7913 \mathrm{E}+00$
$2.7942 \mathrm{E}+00$
$2.7970 \mathrm{E}+00$
$2.7999 \mathrm{E}+00$ 
$2.8028 \mathrm{E}+00$
$2.8056 \mathrm{E}+00$
$2.8085 \mathrm{E}+00$
$2.8113 \mathrm{E}+00$
$2.8142 \mathrm{E}+00$
$2.8171 \mathrm{E}+00$
$2.8199 \mathrm{E}+00$
$2.8228 \mathrm{E}+00$
$2.8256 \mathrm{E}+00$
$2.8285 \mathrm{E}+00$
$2.8314 \mathrm{E}+00$
$2.8342 \mathrm{E}+00$
$2.8371 \mathrm{E}+00$
$2.8399 \mathrm{E}+00$
$2.8428 \mathrm{E}+00$
$2.8456 \mathrm{E}+00$
$2.8485 \mathrm{E}+00$
$2.8514 \mathrm{E}+00$
$2.8542 \mathrm{E}+00$
$2.8571 \mathrm{E}+00$
$2.8599 \mathrm{E}+00$
$2.8628 \mathrm{E}+00$
$2.8656 \mathrm{E}+00$
$2.8685 \mathrm{E}+00$
$2.8713 \mathrm{E}+00$
$2.8742 \mathrm{E}+00$
$2.8771 \mathrm{E}+00$
$2.8799 \mathrm{E}+00$
$2.8828 \mathrm{E}+00$
$2.8856 \mathrm{E}+00$
$2.8885 \mathrm{E}+00$
$2.8913 \mathrm{E}+00$
$2.8942 \mathrm{E}+00$
$2.8970 \mathrm{E}+00$
$2.8999 \mathrm{E}+00$
$2.9027 \mathrm{E}+00$
$2.9056 \mathrm{E}+00$
$2.9084 \mathrm{E}+00$
$2.9113 \mathrm{E}+00$
$2.9141 \mathrm{E}+00$
$2.9170 \mathrm{E}+00$
$2.9198 \mathrm{E}+00$
$2.9227 \mathrm{E}+00$
$2.9255 \mathrm{E}+00$
$2.9284 \mathrm{E}+00$
$2.9312 \mathrm{E}+00$
$2.9341 \mathrm{E}+00$
$2.9369 \mathrm{E}+00$
$2.9398 \mathrm{E}+00$
$2.9426 \mathrm{E}+00$
$2.9455 \mathrm{E}+00$
$2.9483 \mathrm{E}+00$
$2.9512 \mathrm{E}+00$
$2.9540 \mathrm{E}+00$
$2.9569 \mathrm{E}+00$
$2.9597 \mathrm{E}+00$ 


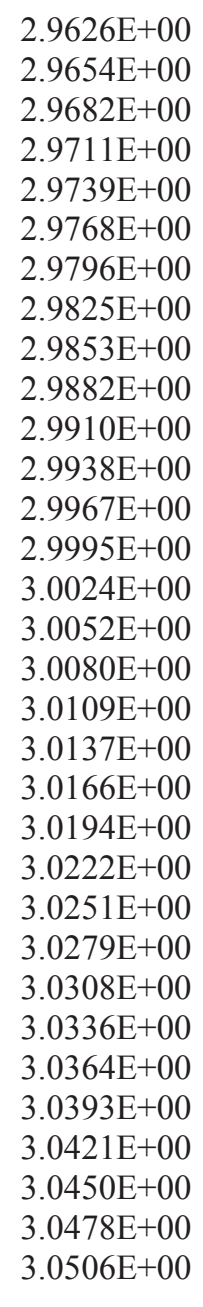

C

FT18 GEB -.0001827 0.0271209 .7812452 


\section{REFERENCES}

1 Phillips, J. R.; et al., Application of Nondestructive Gamma-ray and Neutron Techniques for the Safeguarding of Irradiated Fuel Materials; LA-8212, Los Alamos N.M., 1980.

2 Reilly, D.; Ensslin, N.; Smith, H.; Kreiner, S. Passive Nondestructive Assay of Nuclear Materials; NUREG/CR-5550 (LA-UR-90-732), Los Alamos N.M., 1991.

3 Gerlach D. C.; et al. Determination of the Light Water Reactor Fuel Burnup with Isotope Ratio Method; PNNL-17053; Richland, WA, 2007.

4 Ramalho, A. J. G.; Payne, W. E. Spent Fuel Measurements using High Resolution Gamma Systems. Nuclear Materials Management, 1979, 76-82.

5 Park, K. J.; et al. Determination of Burnup and PU/U Ratio of PWR Spent Fuels by Gamma-Ray Spectrometry. Nuclear Engineering and Technology, 2009, Vol 41, No.10, 1307-1314.

6 Lebrun, A.; Bignan, G. Nondestructive Assay of Nuclear Low-Enriched Uranium Spent Fuels for Burnup Credit. Nuclear Technology, 2001, 135, 216-228.

7 Matsson, I.; Grapengiesser, B.; Andersson, Locket, B.; A gamma-ray spectroscopy system for in-pool measurements of thermal power distribution in irradiated nuclear fuel, Nucl. Instr. and Meth. A, 2006, Vol. 569, Issue 3, 872-882.

8 Genghua, S.; Zeng, Z.; Cheng, Z. Monte Carlo Simulation of In situ $\mathrm{LaBr}_{3}$ GammaRay Spectrometer for Marine Environmental Monitoring. Radiation Protection Dosimetry 2011, 146, 1-3, 103- 106.

9 Skidmore, M.S; Ambrosi R.M.; Simon, H. Hybrid X-ray and g-ray spectrometer for in situ planetary science missions, Nuclear Instruments and Methods in Physics Research 2009, Vol A, 592-604.

10 Kastlander, J.; Bargholtz C. In situ method to determine depth distribution of buried activity,Appl. Radiat. Isot. 2011, 69, 773-779. 
11 D.H. Beddingfield, et al, High-Pressure Xenon Ion Chambers for Gamma-Ray Spectroscopy in Nuclear Safeguards, Nuclear Instruments and Methods in Physics Research 2003, 505, 474-477.

12 Terremoto, L.A.; Zeituni, C.A.; Perrotta, J.A.; Da Silva, J.E. Gamma-ray spectroscopy on irradiated MTR fuel elements, Nucl. Instr. and Meth. 2000, 450, 495-514.

13 Oeda, K.; Hirofumi,H.; Hirota, M. ; Natsume, K.; Kumanomido, H. Calibration of Burnup Monitor Installed in Rokkaso Reprocessing Plant. Jounal of Nuclear Science and Technology, 2000, Vol. 37, No. 6,543-547.

14 Pereda, C.B.; Henríquez, C.A.; Klein, J.D.; Medel, J.R. "Burn up Measurements of LEU Fuel for short cooling Times. J. Phys.: Conf. Ser 2008,134.

15 Burra, T.L.; Charltonb,W.S.; Nakhleh, C.W. Assessing confidence in inferring reactor type and fuel burnup: A Markov chain Monte Carlo approach, Nucl. Instr. and Meth. A, 2005, Vol. 555, Issues 1-2, pp. 426-434.

16 Advanced Test Reactor National Scientific User Facility User's Guide; INL/EXT08-14709; Idaho Falls, ID., 2009

17 Nigg, D. W; et al. Advanced Test Reactor Core Modeling Update Project Annual Report for Fiscal Year 2010; INL/EXT-10-19940; Idaho Falls, ID, 2010.

18 Martens, C.; De Lellis C.; VanPut, P.;Tondeur, F. MCNP simulation and Spectrum Unfolding for an NaI monitor of Radiactivity in Aquatic Systems. Nuclear Instruments and Methods in Physics Research 2007, 580, 118-122, 2007.

19 Sukosd, Cs.; Galster, W.; Licolt, I.;Simonart, M.P. Spectrum Unfolding in High Energy Gamma-Ray Detection with Scintillation Detectors. Nuclear Instruments and Methods in Physics Research 1995, 335, 552-558.

20 Hakimabad, H. M., et al. Evaluation the Nonlinear Response Function of a3 x 3 in NaI Scintillation Detector for PGNAA Applications. Applied Radiation and Isotopes, 2007, Vol 65, 918-926.

21 Zeballos-Chavez, J.Y.; et al. Response Function of a Germanium Detector to Photon Energies Between 6 and $120 \mathrm{KeV}$. Nuclear Instruments and Methods in Physics Research, 2001, Vol 457, 212-219.

22 Kovaltchouck, V.; Machrafi, R. MonteCarlo Simulations of response for Gas Filled and Scintillator detectors with MCNPX Code. Annals of Nuclear Energy, 2011, Vol. $38,788-793$. 
23 Meng, L.J.; Ramsden, D. An Inter-comparison of Three Spectral-Deconvolution Algorithms for Gamma-ray Spectroscopy, IEEE Transactions on Nuclear Science, 2000, Vol. 47, No. 4, 1329-1336.

24 Shepp, L.A.; Vardi, Y.; Maximum Likelihood Reconstruction for Emission Tomography. IEEE Trans. Medical Imaging, 1982, Vol 1(2), 113-122. 